TRANSACTIONS OF THE

AMERICAN MATHEMATICAL SOCIETY

Volume 365, Number 6, June 2013, Pages 2977-3018

S 0002-9947(2012)05660-1

Article electronically published on December 13, 2012

\title{
EXTENSIONS OF EMBEDDINGS BELOW COMPUTABLY ENUMERABLE DEGREES
}

\author{
ROD DOWNEY, NOAM GREENBERG, ANDREW LEWIS, AND ANTONIO MONTALBÁN
}

ABStRACT. Toward establishing the decidability of the two-quantifier theory of the $\Delta_{2}^{0}$ Turing degrees with join, we study extensions of embeddings of upper-semi-lattices into the initial segments of Turing degrees determined by computably enumerable sets, in particular, the degree of the halting set $\mathbf{0}^{\prime}$. We obtain a good deal of sufficient and necessary conditions.

\section{Contents}

1. Introduction

2. Almost contiguity and the global anti-cupping property

3. The generalized anti-cupping condition

4. Joins that imply other joins

5. A multi-generic set

6. No least join

7. The join property below non-generalized-low 2 degrees

References

\section{INTRODUCTION}

Since the introduction of the structure of the Turing degrees $\mathcal{D}$ by Kleene and Post KP54, one of the main interests of computability theory has been to understand its order-theoretic and algebraic properties; this pursuit was extended to many other degree structures as well. Particular attention was paid to countable classes of Turing degrees, with the ordering inherited from $\mathcal{D}$. These are usually classes which consist of the degrees of sets which are definable in arithmetic by formulas of some fixed complexity. For example, classes which were investigated extensively were the classes of computably enumerable degrees, of arithmetic degrees, and of hyper-arithmetic degrees.

In this paper we concentrate on another important such collection, which is also a principal initial segment of this structure: the upper-semi-lattice of the degrees computable from the greatest c.e. degree $\mathbf{0}^{\prime}$, which we denote by $\mathcal{D}_{\left(\leqslant \mathbf{0}^{\prime}\right)}$. The sets that are computable in $\mathbf{0}^{\prime}$ are the $\Delta_{2}^{0}$-definable sets. They form a very natural

Received by the editors December 9, 2009 and, in revised form, June 30, 2011.

2010 Mathematics Subject Classification. Primary 03D28; Secondary 03D25.

Key words and phrases. Turing degrees, decidability, extensions of embeddings.

The first two authors were supported by the Marsden Fund of New Zealand.

The third author was supported by a Royal Society University Research Fellowship.

The fourth author was partially supported by NSF Grant DMS-0600824, and by the Marsden Fund of New Zealand via a postdoctoral fellowship. 
class of sets, one of the reasons being that they are exactly the sets that can be computably approximated [Sho59].

Extensions of embeddings. Much is known about the upper-semi-lattice $\mathcal{D}_{\left(\leqslant 0^{\prime}\right)}$, but we are far from having a clear understanding of what the structure really looks like. We do know it is a complicated structure; for instance, we know that its theory is undecidable Eps79, Ler83. Moreover, its theory is one-to-one equivalent to true first order arithmetic (Shore Sho81]). On the other hand, if we look only at existential sentences, we can decide which such sentences hold in $\mathcal{D}_{\left(\leqslant \mathbf{0}^{\prime}\right)}$ : every existential sentence that does not obviously contradict the axioms of upper-semilattices holds in $\mathcal{D}_{\left(\leqslant \mathbf{0}^{\prime}\right)}$ (this follows from results in KP54]). In other words, the one-quantifier theory of $\mathcal{D}_{\left(\leqslant \mathbf{0}^{\prime}\right)}$ is decidable.

In order to understand where the complexity of a certain structure lies, one natural question to ask is what fragments of its theory are decidable. It has always been the case that answers to this question, by exposing either decidability procedures or coding methods, have given us a good deal of information about the algebraic properties of the structure. In Figure 1 we show the results known so far for $\mathcal{D}_{\left(\leqslant \mathbf{0}^{\prime}\right)}$. We note that when dealing with fragments which are determined by few quantifier alterations, it makes sense to enrich the structure by functions and relations which are definable, but not by quantifier-free formulas. The work in this paper is oriented towards addressing the one question-mark left in the table: whether the two-quantifier theory of $\mathcal{D}_{\left(\leqslant \mathbf{0}^{\prime}\right)}$, expanded by adding the join (least upper bound) operation, is decidable or not.

These investigations have also been undertaken for the whole structure $\mathcal{D}$ of the Turing degrees, for the structure $\mathcal{R}$ of the computably enumerable degrees, and for many other structures. We refer the reader to [Sho06] for a recent survey of known results.

Decidability results of $\exists$-theories and $\forall \exists$-theories are closely related to embeddability results. Given a finite relational language $\mathcal{L}$ and an $\mathcal{L}$-structure $\mathcal{A}$, the existential theory $\exists \operatorname{Th}_{\mathcal{L}}(\mathcal{A})$ is decidable if and only if the set of finite $\mathcal{L}$-structures $\mathcal{P}$ which embed into $\mathcal{A}$ is computable; the fragment $\forall \exists \mathrm{Th}_{\mathcal{L}}(\mathcal{A})$ is decidable if and only if given a finite tuple of $\mathcal{L}$-structures $\left(\mathcal{P}, \mathcal{Q}_{1}, \ldots, \mathcal{Q}_{m}\right)$ with $\mathcal{P} \subseteq \mathcal{Q}_{i}$ for all $i \leqslant m$, it is decidable whether every embedding $\mathcal{P} \hookrightarrow \mathcal{A}$ has an extension $\mathcal{Q}_{i} \hookrightarrow \mathcal{A}$ for some $i$. The extensions-of-embeddings problem for $\mathcal{A}$ is the restriction of this latter problem to the case $m=1$. Hence, in terms of computational complexity, the extensions-of-embedding problem for $\mathcal{A}$ lies between the $\exists$-theory and the $\forall \exists$-theory of $\mathcal{A}$.

For a recent survey of embeddability results in the Turing degrees, see [Mon].

Definition 1.1. An upper-semi-lattice (usl) is a partial ordering in which every pair of elements has a least upper bound. We denote the least upper bound of a and $\mathbf{b}$ by $\mathbf{a} \vee \mathbf{b}$. All the usls we consider will have a top element $\mathbf{1}$ and a bottom element $\mathbf{0}$. A usl embedding has to preserve not only the ordering, non-ordering, and join operation, but also the top and bottom elements. When we write $\mathcal{P} \subseteq \mathcal{Q}$ we mean that the top and bottom elements of $\mathcal{P}$ and $\mathcal{Q}$ coincide, that is, the identity on $\mathcal{P}$ is a usl embedding into $\mathcal{Q}$.

Let $\mathbb{E}$ be the set of pairs of usls $(\mathcal{P}, \mathcal{Q})$, such that $\mathcal{P} \subseteq \mathcal{Q}$ and such that every usl embedding of $\mathcal{P}$ into $\left\langle\mathcal{D}_{\left(\leqslant \mathbf{0}^{\prime}\right)}, \leqslant, \vee, \mathbf{0}, \mathbf{0}^{\prime}\right\rangle$ can be extended to an embedding of $\mathcal{Q}$ into $\left\langle\mathcal{D}_{\left(\leqslant \mathbf{0}^{\prime}\right)}, \leqslant, \vee, \mathbf{0}, \mathbf{0}^{\prime}\right\rangle$. Thus $\mathbb{E}$ is the extensions-of-embeddings problem for $\mathcal{D}_{\left(\leqslant \mathbf{0}^{\prime}\right)}$. 


\begin{tabular}{l|lll} 
& $\exists$ & $\forall \exists$ & $\exists \forall \exists$ \\
\hline$\left(\mathcal{D}_{\left(\leqslant \mathbf{0}^{\prime}\right)}, \leqslant\right)$ & decidable & decidable & undecidable \\
$\left(\mathcal{D}_{\left(\leqslant \mathbf{0}^{\prime}\right)}, \leqslant, \vee\right)$ & decidable & $?$ & undecidable \\
$\left(\mathcal{D}_{\left(\leqslant \mathbf{0}^{\prime}\right)}, \leqslant, \vee, \wedge\right)$ & decidable & undecidable & undecidable
\end{tabular}

Figure 1. The decidability of $\exists \operatorname{Th}\left(\mathcal{D}_{\left(\leqslant \mathbf{0}^{\prime}\right)}, \leqslant, \vee\right)$ follows from the work of Kleene and Post KP54. The decidability of $\exists \operatorname{Th}\left(\mathcal{D}_{\left(\leqslant \mathbf{0}^{\prime}\right)}, \leqslant, \vee, \wedge\right)$, where $\wedge$ is the partial binary operation that is the greatest lower bound operation, follows from the Lachlan and Lebeuf lattice embedding theorem [LL76]. The undecidability of $\exists \forall \exists \operatorname{Th}\left(\mathcal{D}_{\left(\leqslant \mathbf{0}^{\prime}\right)}, \leqslant\right)$ is due to Schmerl and Lerman Ler83. The decidability of $\forall \exists \operatorname{Th}\left(\mathcal{D}_{\left(\leqslant \mathbf{0}^{\prime}\right)}, \leqslant\right)$ is due to Lerman and Shore [LS88. The undecidability of $\forall \exists \operatorname{Th}\left(\mathcal{D}_{\left(\leqslant 0^{\prime}\right)}, \leqslant, \vee, \wedge\right)$, where $\wedge$ is any total binary operation that is the greatest lower bound operation when this exists, is due to R. Miller, Nies and Shore [MNS04.

In order to find a procedure for deciding $\forall \exists \operatorname{Th}\left(\mathcal{D}_{\left(\leqslant \mathbf{0}^{\prime}\right)}, \leqslant, \vee\right)$ we definitely have to start by solving the extensions-of-embeddings problem for this structure, that is, by showing that $\mathbb{E}$ is a computable set.

Conversely, in some occasions, deciding the extensions-of-embeddings problem has been sufficient to show the decidability of $\forall \exists$-theories. This was the case with the decidability of $\forall \exists \operatorname{Th}(\mathcal{D}, \leqslant, \vee)$ by Jockusch and Slaman JS93 and the decidability of $\forall \exists \operatorname{Th}\left(\mathcal{D}_{\left(\leqslant \mathbf{0}^{\prime}\right)}, \leqslant_{T}\right)$ by Lerman and Shore [LS88. The extension-of-embedding problem for $\left(\mathcal{R}, \leqslant_{T}\right)$, proved decidable by Slaman and Soare [SS01, was the first one whose decision procedure was not trivial. This result did not produce a decision procedure for $\forall \exists \operatorname{Th}(\mathcal{R}, \leqslant)$. We expect a similar behavior for $\mathcal{D}_{\left(\leqslant \mathbf{0}^{\prime}\right)}$, in the sense that solving the extension-of-embeddings problem for $\mathcal{D}_{\left(\leqslant \mathbf{0}^{\prime}\right)}$ will not be enough to decide its $\forall \exists$-theory. We will give evidence for this suspicion below.

We have not yet found a decision procedure for the extension-of-embedding problem for $\mathcal{D}_{\left(\leqslant \mathbf{0}^{\prime}\right)}$. However, we have found a good deal of necessary and sufficient conditions that we expect will eventually lead to a solution of the problem. Many of the theorems we proved for this purpose are interesting in their own right, and provide a better understanding of the structure $\mathcal{D}_{\left(\leqslant \mathbf{0}^{\prime}\right)}$.

Known results. Let us start analyzing whether $(\mathcal{P}, \mathcal{Q}) \in \mathbb{E}$ for the simplest cases. Suppose $\mathcal{P}=\{\mathbf{0}<\mathbf{a}<\mathbf{1}\}$ is a 3 -element chain, and that $\mathcal{Q} \supset \mathcal{P}$ is generated from $\mathcal{P}$ by adding a single element $\mathbf{x}$; we write $\mathcal{Q}=\mathcal{P}[\mathbf{x}]$. We identify $\mathcal{P}$ with its image under an embedding of $\mathcal{P}$ into $\mathcal{D}_{\left(\leqslant \mathbf{0}^{\prime}\right)}$, so $\mathbf{1}=\mathbf{0}^{\prime}$. There are four different possibilities for $\mathcal{Q}$.
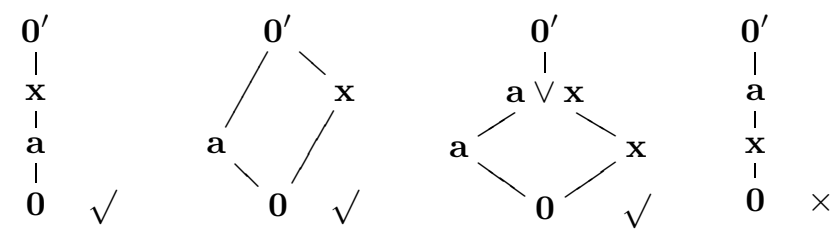

First, if $\mathbf{a}<\mathbf{x}<\mathbf{1}$, since $\mathbf{0}^{\prime}$ is c.e. over $\mathbf{a}$, by the downwards density of the c.e. degrees (Sacks Splitting Theorem [Sac63]), we know that $\mathbf{0}^{\prime}$ is not minimal over a. 
Therefore, there is a degree $\mathbf{x}$ as wanted, and every embedding of $\mathcal{P}$ into $\mathcal{D}_{\left(\leqslant \mathbf{0}^{\prime}\right)}$ can be extended to an embedding of $\mathcal{Q}$ into $\mathcal{D}_{\left(\leqslant \mathbf{0}^{\prime}\right)}$.

Second, suppose that $\mathbf{a}$ and $\mathbf{x}$ are incomparable and $\mathbf{x} \vee \mathbf{a}=\mathbf{1}$. In this case, the extension is possible by the following theorem.

Theorem 1.2 (Robinson Rob72, Posner and Robinson PR81]). For every degree $\mathbf{a}<\mathbf{0}^{\prime}$, there exists $\mathbf{x}<\mathbf{0}^{\prime}$ such that $\mathbf{a} \vee \mathbf{x}=\mathbf{0}^{\prime}$.

Third, comes the case where $\mathbf{a}$ and $\mathbf{x}$ are incomparable but $\mathbf{x} \vee \mathbf{a}<\mathbf{1}$. Since $\mathbf{0}^{\prime}$ is c.e. over $\mathbf{a}$, there is a 1-a-generic degree $\mathbf{x}$ below $\mathbf{0}^{\prime}$ (see [Soa87, Ex. VI 3.9]). Therefore, $\mathbf{x}$ is incomparable to $\mathbf{a}$ and $\mathbf{x} \vee \mathbf{a}$ does not compute $\mathbf{0}^{\prime}$ (as it does not compute any a-c.e. set). So the extension is possible.

The last case is $\mathbf{0}<\mathbf{x}<\mathbf{a}$. In this case the extension will not be possible when $\mathbf{a}$ is a minimal degree, and we know there are minimal degrees below $\mathbf{0}^{\prime}$ (Sacks Sac61]). The analysis for this last case can be extended to a much more general setting:

Definition 1.3. If $\mathcal{P} \subseteq \mathcal{Q}$ are usls, we say that $\mathcal{Q}$ is an end extension of $\mathcal{P}$, if no element of $\mathcal{Q} \backslash \mathcal{P}$ lies below an element of $\mathcal{P}$, with the obvious exception of $\mathbf{1}$.

Lemma 1.4. If $(\mathcal{P}, \mathcal{Q}) \in \mathbb{E}$, then $\mathcal{Q}$ is an end extension of $\mathcal{P}$.

Proof. Suppose $\mathcal{Q}$ is not an end extension of $\mathcal{P}$. An embedding of $\mathcal{P}$, where $\mathcal{P} \backslash \mathbf{1}$ is an initial segment below $\mathbf{0}^{\prime}$, would not have an extension to $\mathcal{Q}$. The existence of such an embedding of $\mathcal{P}$ was proved by Lerman and Shore [LS88. So, $(\mathcal{P}, \mathcal{Q}) \notin \mathbb{E}$.

There is another necessary condition for extension-of-embeddings of a different nature that follows from known results. Here is the key theorem.

Theorem 1.5 (Cooper [Coo89], Slaman, Steel [SS89]). There are c.e. degrees $\mathbf{0}<$ $\mathbf{b}<\mathbf{c}$ such that for no $\mathbf{x}<\mathbf{c}$ do we have $\mathbf{b} \vee \mathbf{x}=\mathbf{c}$.

We say that $\mathbf{c}$ fails the join property, witnessed by b. Using Jockusch and Shore's pseudo-jump inversion theorem JS83, we obtain a c.e. degree a, relative to which $\mathbf{0}^{\prime}$ fails the join property: there is some $\mathbf{b}$, strictly between $\mathbf{a}$ and $\mathbf{0}^{\prime}$, such that there is no $\mathbf{x}$ strictly between $\mathbf{a}$ and $\mathbf{0}^{\prime}$ such that $\mathbf{b} \vee \mathbf{x}=\mathbf{0}^{\prime}$.

Therefore, if $\mathcal{P}=\{\mathbf{0}<\mathbf{a}<\mathbf{b}<\mathbf{1}\}$ and $\mathcal{Q}=\mathcal{P}[\mathbf{x}]$, where $\mathbf{a}<\mathbf{x}<\mathbf{1}$ and $\mathbf{b} \vee \mathbf{x}=\mathbf{1}$, then $(\mathcal{P}, \mathcal{Q}) \notin \mathbb{E}$.

We will see that if $(\mathcal{P}, \mathcal{Q}) \in \mathbb{E}$, then the configuration we just described cannot appear inside $(\mathcal{P}, \mathcal{Q})$ in a sense we will specify later. We will also extend Theorem 1.5 and get other necessary conditions to have the extensions-of-embeddings property.

The $\forall \exists$-theory. When Jockusch and Slaman [JS93. proved the decidability of $\forall \exists \operatorname{Th}(\mathcal{D}, \leqslant, \vee$ ), they proved that a pair of usls $\mathcal{P}$ and $\mathcal{Q}$ (with a bottom element, but without a top element) has the extensions-of-embedding property if and only if $\mathcal{Q}$ is an end extension of $\mathcal{P}$. The fact that this condition is necessary follows from the fact that any usl can be embedded as an initial segment of the Turing degrees. It then follows that given $\left(\mathcal{P}, \mathcal{Q}_{1}, \ldots, \mathcal{Q}_{k}\right)$ with $\mathcal{P} \subseteq \mathcal{Q}_{i}$, we have that every embedding of $\mathcal{P}$ into $(\mathcal{D}, \leqslant, \vee)$ has an extension to some $\mathcal{Q}_{i}$ if and only if some $\mathcal{Q}_{i}$ is an end extension of $\mathcal{P}$. Hence, $\forall \exists \operatorname{Th}(\mathcal{D}, \leqslant, \vee)$ is decidable. A very similar behavior occurred with Lerman and Shore's proof of the decidability of $\forall \exists \operatorname{Th}\left(\mathcal{D}_{\left(\leqslant \mathbf{0}^{\prime}\right)}, \leqslant T\right)$. 
The following example shows that proving the decidability of $\forall \exists \operatorname{Th}\left(\mathcal{D}_{\left(\leqslant \mathbf{0}^{\prime}\right)}, \leqslant_{T}, \vee\right)$ will require more work than just solving the extensions-of-embedding problem.

Example 1.6 (Montalbán [Mon]). Let $\mathcal{P}=\{\mathbf{0}<\mathbf{a}<\mathbf{b}<\mathbf{1}\}$; let $\mathcal{Q}_{1}$ be the one-element extension $\mathcal{P}[\mathbf{x}]$, where $\mathbf{0}<\mathbf{x}<\mathbf{a}$; let $\mathcal{Q}_{2}$ be the one-element extension $\mathcal{P}[\mathbf{x}]$, where $\mathbf{a}<\mathbf{x}<\mathbf{1}$ and $\mathbf{x} \vee \mathbf{b}=\mathbf{1}$. See Figure 2, By our observations above, we see that there is an embedding of $\mathcal{P}$ which has no extensions to $\mathcal{Q}_{1}$, namely the one where $\mathbf{a}$ is a minimal degree. Also, there is an embedding of $\mathcal{P}$ which has no extension to $\mathcal{Q}_{2}$, namely the one we obtained by inverting the pseudo-jump operator given by the Cooper or the Slaman-Steel constructions, making $\mathbf{0}^{\prime}$ fail the join property relative to $\mathbf{a}$, as witnessed by $\mathbf{b}$. However, every embedding of $\mathcal{P}$ into $\mathcal{D}_{\left(\leqslant \mathbf{0}^{\prime}\right)}$ can be extended to either $\mathcal{Q}_{1}$ or $\mathcal{Q}_{2}$ : On the one hand, if a is not minimal, then we can extend to $\mathcal{Q}_{1}$; on the other hand, if $\mathbf{a}$ is minimal, then $\mathbf{a}$ is $\mathrm{low}_{2}$, and hence $\mathbf{0}^{\prime}$ is high over $\mathbf{a}$, and hence we can get $\mathbf{x}$ by Posner's Pos77. join theorem. Posner's Theorem is the generalization of Theorem 1.2 to any high degree in place of $\mathbf{0}^{\prime}$.
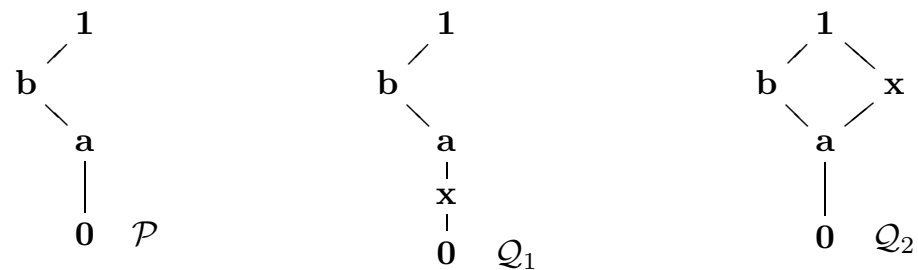

Figure 2. The $\forall \exists \operatorname{Th}\left(\mathcal{D}_{\left(\leqslant \mathbf{0}^{\prime}\right)}, \leqslant, \vee\right)$ is not immediately computable from the extensions-of-embeddings problem.

We show in this paper how Posner's Theorem and Theorem 1.2 can be extended to any non-low 2 degree, furthermore, to any non-generalized-low 2 degree.

Theorem 1.7. Let $\mathbf{c}$ be a non-GL $L_{2}$ degree. Then for every non-zero degree $\mathbf{a}<\mathbf{c}$ there is some $\mathbf{x}<\mathbf{c}$ such that $\mathbf{a} \vee \mathbf{x}=\mathbf{c}$.

We will prove this theorem in Section 7 using ideas from Slaman and Steel's SS89] uniform proof of the join theorem for $\mathbf{0}^{\prime}$. We believe that this theorem, and maybe other theorems regarding non-low 2 degrees, will be important to solve the decidability of $\forall \exists \operatorname{Th}\left(\mathcal{D}_{\left(\leqslant \mathbf{0}^{\prime}\right)}, \leqslant, \vee\right)$, as illustrated in the following example.

Example 1.8. Let $\mathcal{P}=\left\{\mathbf{0}=\mathbf{a}_{0}<\mathbf{b}_{1}<\mathbf{a}_{1}<\mathbf{b}_{2}<\ldots<\mathbf{a}_{n}=\mathbf{1}\right\}$; for each $i=1, \ldots, n$, let $\mathcal{Q}_{i}$ be the one-element extension $\mathcal{P}[\mathbf{x}]$, where $\mathbf{a}_{i-1}<\mathbf{x}<\mathbf{a}_{i}$ and $\mathbf{b}_{i} \vee \mathbf{x}=\mathbf{a}_{i}$. See Figure 3, which illustrates the case $n=3$. Then, as in the previous example, we can show that for each $i$, there is an embedding of $\mathcal{P}$ which has no extension to $\mathcal{Q}_{i}$. However, for every embedding of $\mathcal{P}$ there is some $i$ such that the embedding extends to $\mathcal{Q}_{i}$. The reason is that for some $i$ we have to have that $\mathbf{a}_{i}$ is non-low 2 over $\mathbf{a}_{i-1}$, because otherwise $\mathbf{0}^{\prime}=\mathbf{a}_{n}$ would be low 2 over $\mathbf{0}=\mathbf{a}_{0}$. Then we apply Theorem 1.7 relative to $\mathbf{a}_{i-1}$. 

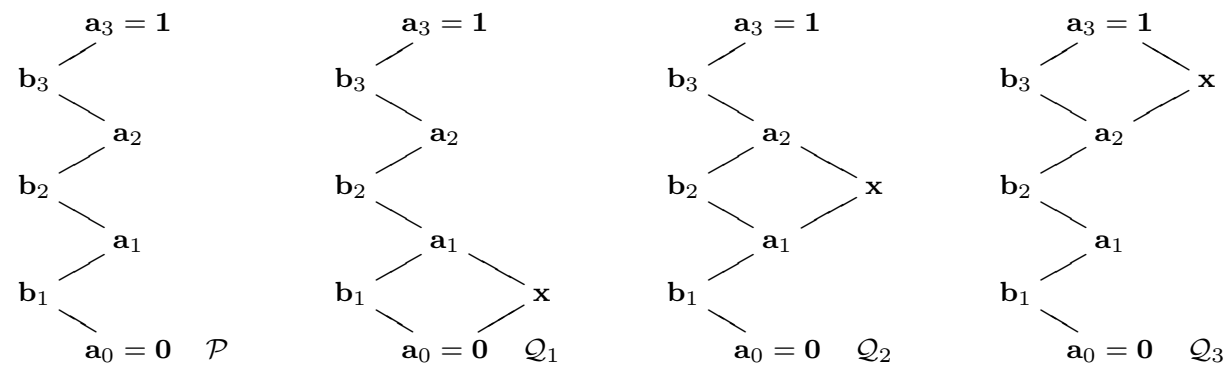

FiguRE 3. Example 1.8

Extensions below a c.e. set. In computability theory, most of the proofs are relativizable, and one would expect that the solution of the extension-of-embeddings problem (if decidable) will also be relativizable. So, it makes sense to study the following extension-of-embeddings set, as it is possible it might end up being equal to $\mathbb{E}$.

Definition 1.9. Let $\mathbb{E}^{\text {jump }}=\{(\mathcal{P}, \mathcal{Q})$ finite usls: every embedding $h: \mathcal{P} \hookrightarrow \mathcal{D}$ with $h(\mathbf{1}) \equiv_{T} h(\mathbf{0})^{\prime}$ has an extension to $\left.\mathcal{Q} \hookrightarrow \mathcal{D}\right\}$.

The method of pseudo-jump inversion used in Example 1.6 suggests that it will also be useful to study the extension-of-embeddings problem below any c.e. degree: essentially, given any c.e. degree $\mathbf{c}$, there is some degree $\mathbf{a}<\mathbf{0}^{\prime}$ such that relative to $\mathbf{a}, \mathbf{0}^{\prime}$ behaves like $\mathbf{c}$.

Definition 1.10. Let $\mathbb{E}^{\text {c.e. }}=\{(\mathcal{P}, \mathcal{Q})$ finite usls: every embedding $h: \mathcal{P} \hookrightarrow \mathcal{D}$ where $h(\mathbf{1})$ is c.e. in $h(\mathbf{0})$ has an extension to $\mathcal{Q} \hookrightarrow \mathcal{D}\}$.

It is not hard to see that

$$
(\mathcal{P}, \mathcal{Q}) \in \mathbb{E}^{\text {c.e. }} \Longrightarrow(\mathcal{P}, \mathcal{Q}) \in \mathbb{E}^{\text {jump }} \Longrightarrow(\mathcal{P}, \mathcal{Q}) \in \mathbb{E} .
$$

The first of these implications cannot be reversed: take $\mathcal{P}=\{\mathbf{0}<\mathbf{a}<\mathbf{1}\}$ and $\mathcal{Q}=\mathcal{P}[\mathbf{x}]$, where $\mathbf{a} \vee \mathbf{x}=\mathbf{1}$. By Theorem 1.5 , $(\mathcal{P}, \mathcal{Q}) \notin \mathbb{E}^{\text {c.e. }}$, but, using a relativized form of Theorem 1.2 , we get $(\mathcal{P}, \mathcal{Q}) \in \mathbb{E}^{\text {jump }}$.

However, there is a restatement of the implication above that might be reversible. Given a usl $\mathcal{P}$, let $\mathcal{P}_{*}$ be $\mathcal{P} \cup\left\{\mathbf{0}_{*}\right\}$, where $\mathbf{0}_{*}$ is strictly below all the elements of $\mathcal{P}$. If $\mathbb{E}^{\text {c.e. }}, \mathbb{E}^{\text {jump }}$ and $\mathbb{E}$ are decidable, and proofs are relativizable, the pseudo-jump inversion technique would lead us to expect the following equivalence:

$$
(\mathcal{P}, \mathcal{Q}) \in \mathbb{E}^{\text {c.e. }} \Longleftrightarrow\left(\mathcal{P}_{*}, \mathcal{Q}_{*}\right) \in \mathbb{E}^{j u m p} \Longleftrightarrow\left(\mathcal{P}_{*}, \mathcal{Q}_{*}\right) \in \mathbb{E} .
$$

We thus believe that understanding $\mathbb{E}^{\text {c.e. }}$ is a key to understanding $\mathbb{E}$. The rest of the paper is dedicated to the study of $\mathbb{E}^{\text {c.e. }}$.

Necessary conditions. We have already shown that if $(\mathcal{P}, \mathcal{Q}) \in \mathbb{E}$, then $\mathcal{Q}$ is an end extension of $\mathcal{P}$. Since $\mathbb{E}^{\text {c.e. }} \subseteq \mathbb{E}$, the same holds for $\mathbb{E}^{\text {c.e. }}$. So, from now on, we will always assume that $\mathcal{Q}$ is an end extension of $\mathcal{P}$.

The other negative extension-of-embeddings result we have mentioned is Theorem 1.5. We would like to get a result saying that if $(\mathcal{P}, \mathcal{Q})$ contains a configuration similar to the one of that theorem, then $(\mathcal{P}, \mathcal{Q}) \notin \mathbb{E}^{\text {c.e. }}$.

The first extension of Theorem 1.5 that we obtain is the following one. 
Theorem 1.11. There are c.e. degrees $\mathbf{a}, \mathbf{b}$ and $\mathbf{c}$ such that $\mathbf{0}<\mathbf{a}<\mathbf{b}<\mathbf{c}$, and for every $\mathbf{x} \leqslant \mathbf{c}$, if $\mathbf{b} \nless \mathbf{x}$, then $\mathbf{b} \nless \mathbf{x} \vee \mathbf{a}$. That is, in $\mathcal{D}_{(\leqslant \mathbf{c})}$, no degree non-trivially joins $\mathbf{a}$ above $\mathbf{b}$.

This theorem follows from the following two lemmas, which use a notion similar to contiguity. Recall that a degree $\mathbf{a}$ is strongly contiguous if any two sets $A, B \in \mathbf{a}$ are weak truth-table equivalent. This notion was defined by Downey [Dow87, based on work by Ladner and Sasso [LS75]. We will not use contiguous degrees, but rather, the similar notion of a contiguous pair, which is a pair as in the following lemma. We will prove this lemma in Subsection 2.1.

Lemma 1.12. There exist c.e. sets $B$ and $C$ such that $\emptyset<_{T} B<_{T} C$, and such that for every set $X$, if $B \leqslant_{T} X \leqslant_{T} C$, then $B \leqslant_{w t t} X$.

Then, we will use the global anti-cupping theorem for weak truth-table degrees.

Lemma 1.13 (Downey Dow87]). For every non-computable c.e. set B, there exists a non-computable c.e. set $A<_{T} B$ such that for every set $X$,

$$
X \oplus A \geqslant_{w t t} B \Longrightarrow X \geqslant_{T} B .
$$

To prove Theorem 1.11, let $B$ and $C$ be the sets guaranteed by Lemma 1.12, and let $A$ be the anti-cupping witness for $B$ given by Lemma 1.13, and let $\mathbf{a}=\operatorname{deg}_{T}(A)$, $\mathbf{b}=\operatorname{deg}_{T}(B)$, and $\mathbf{c}=\operatorname{deg}_{T}(C)$.

Now, suppose that $\mathcal{P} \subseteq \mathcal{Q}$ are two usls, and suppose that there are $\mathbf{a}<\mathbf{b}$ in $\mathcal{P}$ and some $\mathbf{x} \in \mathcal{Q} \backslash \mathcal{P}$ which in $\mathcal{Q}$ non-trivially joins a above $\mathbf{b}$; that is, in $\mathcal{Q}, \mathbf{x} \ngtr \mathbf{b}$, but $\mathbf{a} \vee \mathbf{x} \geqslant \mathbf{b}$. We would like to use Theorem 1.11 to deduce that $(\mathcal{P}, \mathcal{Q}) \notin \mathbb{E}^{\text {c.e. }}$. In order to do this, we would like to find an embedding of $\mathcal{P}$ into $\mathcal{D}_{(\leqslant \mathbf{c})}$ for some c.e. degree $\mathbf{c}$, where the images of $\mathbf{a}$ and $\mathbf{b}$ satisfy the non-join property in $\mathcal{D}_{(\leqslant \mathbf{c})}$ as in Theorem 1.11, this would preclude an extension of this embedding to $\mathcal{Q}$. However, this plan is impossible if already in $\mathbb{P}$ there is some $\mathbf{y}$ which non-trivially joins a above $\mathbf{b}$.

Example 1.14. Suppose $\mathcal{P}=\left\{\mathbf{0}<\mathbf{a}_{0}, \mathbf{a}_{1}<\mathbf{b}<\mathbf{1}\right\}$, where $\mathbf{a}_{0} \vee \mathbf{a}_{1}=\mathbf{b}$, and that $\mathcal{Q}=\mathcal{P}[\mathbf{x}]$, where $\mathbf{a}_{0} \vee \mathbf{x}>\mathbf{b}$ and $\mathbf{a}_{1} \vee \mathbf{x}>\mathbf{b}$. As we said above, we will not be able to get an embedding of $\mathcal{P}$ where for no $\mathbf{x} \ngtr \mathbf{b}$ we have that $\mathbf{a}_{0} \vee \mathbf{x}>\mathbf{b}$ because $\mathbf{a}_{1}$ already has this property. However, by merging the proofs of the next lemma and Lemma 1.12 we can get an embedding of $\mathcal{P}$ such that for no $\mathbf{x} \ngtr \mathbf{b}$ we simultaneously have that $\mathbf{a}_{0} \vee \mathbf{x}>\mathbf{b}$ and $\mathbf{a}_{1} \vee \mathbf{x}>\mathbf{b}$, and so nonetheless we get $(\mathcal{P}, \mathcal{Q}) \notin \mathbb{E}^{\text {c.e. }}$. We will merge these proofs and other proofs in Theorem 1.17 .

Lemma 1.15. There exist Turing-incomparable, disjoint c.e. sets $A_{0}$ and $A_{1}$ such that for every set $X \subseteq \omega$,

$$
X \oplus A_{0} \geqslant_{w t t} B \quad \& \quad X \oplus A_{1} \geqslant_{w t t} B \Longrightarrow X \geqslant_{T} B
$$

where $B=A_{0} \cup A_{1}$.

(We recall that $\operatorname{deg}_{T}(B)=\operatorname{deg}_{T}\left(A_{0}\right) \vee \operatorname{deg}_{T}\left(A_{1}\right)$.)

We will prove this lemma in Subsection 2.2, Of course, instead of having $B$ split into two sets $A_{0}$ and $A_{1}$, we could split $B$ into as many sets as we want.

We will exploit Example 1.14 and show that if a similar configuration occurs inside a pair $(\mathcal{P}, \mathcal{Q})$, then $(\mathcal{P}, \mathcal{Q}) \notin \mathbb{E}^{c . e}$. Let us describe this in more detail. 
Definition 1.16. Let $\mathcal{P}$ be a usl and $\mathcal{Q}$ be an extension of $\mathcal{P}$. We say that $(\mathcal{P}, \mathcal{Q})$ satisfies the anti-cupping condition, and write $(\mathcal{P}, \mathcal{Q}) \models$ ACC, if for every $\mathbf{x} \in \mathcal{Q} \backslash \mathcal{P}$ and for every $\mathbf{b} \in \mathcal{P}$ such that $\mathbf{b} \nless \mathbf{x}$, there exists $\mathbf{c} \in \mathcal{P}$ (c might be $\mathbf{0}$ ) such that $\mathbf{c} \ngtr \mathbf{b}$ and for every $\mathbf{a}<\mathbf{b}$ in $\mathcal{P}$ we have that

$$
\mathbf{x} \vee \mathbf{a} \geqslant \mathbf{b} \Longrightarrow \mathbf{c} \vee \mathbf{a} \geqslant \mathbf{b} .
$$

In Section 3 we will prove the following theorem, which says that this is a necessary condition.

Theorem 1.17. Let $\mathcal{P}$ be a usl, and let $\mathcal{Q}=\mathcal{P}[\mathbf{x}]$ be an extension of $\mathcal{P}$ generated by a single element. If $(\mathcal{P}, \mathcal{Q}) \in \mathbb{E}^{\text {c.e. }}$, then $(\mathcal{P}, \mathcal{Q}) \models \mathrm{ACC}$.

We do not know whether the anti-cupping condition is sufficient for a pair $(\mathcal{P}, \mathcal{P}[\mathbf{x}])$ to be in $\mathbb{E}^{\text {c.e. }}$. We suspect that it is not. The reason for this suspicion is the following theorem.

Theorem 1.18. There exist c.e. sets $A, B, C, D$ and $E$ such that $A, B, D$ and $E$ are all Turing reducible to $C$ and pairwise incomparable, and such that any $\Delta_{2}^{0}$ set $X$ which is computable in $C$ and joins $A$ above $B$ also joins $D$ above $E$.

While Theorem 1.18 does not construct a usl $\mathcal{P}$ and some $\mathcal{Q}=\mathcal{P}[\mathbf{x}]$ satisfying the ACC but failing the extension of embedding property, it hints that such a construction is possible. It does so by showing that in some $\mathcal{D}_{(\leqslant \mathbf{c})}$, not all joins are "independent". We suspect that a modification of Theorem 1.18 may show that the anti-cupping condition is not sufficient for membership in $\mathbb{E}^{\text {c.e. }}$.

Theorem 1.18 says, in a sense, that if $X \leqslant_{T} C$ joins $A$ above $B$, then there is a certain amount of information encoded in $X$, and this information is enough to join $D$ above $E$. The natural question that follows is whether this information can compute some non-zero degree.

Question 1.19. Is there a c.e. set $C$, incomparable sets $A, B<_{T} C$ and a noncomputable set $E$ such that for any $\Delta_{2}^{0}$ set $X$ which is computable in $C$, if $X$ joins $A$ above $B$, then $X$ computes $E$ ?

Sufficient conditions. There are some cases where we know we always have the extensions-of-embeddings property. We start by looking at free extensions.

Definition 1.20. We say that $\mathcal{Q}$ is a free extension of $\mathcal{P}$ if $\mathcal{Q}=\mathcal{P}[F]$ for some finite set $F$, and given $p_{0} \vee \bigvee A_{0}$ and $p_{1} \vee \bigvee A_{1}$ with $p_{0}, p_{1} \in \mathcal{P}$, and $A_{0}, A_{1} \subseteq F$, we have that

$$
p_{0} \vee \bigvee A_{0} \leqslant p_{1} \vee \bigvee A_{1} \Longleftrightarrow p_{0} \leqslant p_{1} \& A_{0} \subseteq A_{1}
$$

Lemma 1.21. Every free extension belongs to $\mathbb{E}^{\text {c.e. }}$.

To prove this lemma it is enough to consider $F$ with one element because if $F=\left\{\mathbf{x}_{1}, \ldots, \mathbf{x}_{k}\right\}$, then $\mathcal{P}[F]=\mathcal{P}\left[\mathbf{x}_{1}\right]\left[\mathbf{x}_{2}\right] \ldots\left[\mathbf{x}_{k}\right]$, where each of these 1-generator extensions is free.

In the case where $\mathcal{P}$ has one element, which we discussed above, we used a 1-ageneric set to get the free extension and the fact that there are 1-generic degrees below any c.e. set. When $\mathcal{P}$ has more elements, we would like to get a set $G$, computable from $\mathbf{1}$, that is, 1-generic relative to all the elements of $\mathcal{P} \backslash\{\mathbf{1}\}$. This set $G$ is easily obtainable if $\mathcal{P} \backslash\{\mathbf{1}\}$ has a maximal element. However, we prove that we can get such a set $G$ even if this is not the case. 
Theorem 1.22. Let $C$ be a c.e. set and let $\left\{A_{i}: i \in \omega\right\}$ be a uniformly $C$ computable list of sets. Then, there exists a set $G \leqslant_{T} C$ such that $G$ is 1-generic relative to $A_{i}$, for every $i \in \omega$ such that $A_{i}<_{T} C$.

Lemma 1.21 follows from Theorem 1.22 using two basic properties of 1-generic sets: 1-generic sets do not compute c.e. degrees, and if $A<_{T} B$ and $G$ is $1-B$ generic, then $A \oplus G \ngtr_{T} B$ [Joc80].

Let us now go back to the extension of Theorems 1.5 and 1.11. Suppose we have $\mathbf{0}<\mathbf{a}<\mathbf{d}<\mathbf{c}$, where $\mathbf{c}$ is c.e., and we want to get $\mathbf{x} \leqslant \mathbf{c}$ such that

$$
\mathbf{d} \not \mathbf{x} \quad \& \quad \mathbf{d} \leqslant \mathbf{x} \vee \mathbf{a} \text {. }
$$

Theorem 1.11 tells us that we might find $\mathbf{a}, \mathbf{d}$ and $\mathbf{c}$ such that no such $\mathbf{x}$ exists. Suppose now that we know there is some $\mathbf{b}<\mathbf{d}$ such that $\mathbf{a} \vee \mathbf{b}=\mathbf{d}$. In this case we can find $\mathbf{x}$ as above just by letting $\mathbf{x}=\mathbf{b}$. However, suppose we do not want to cheat, and we want to get such an $\mathbf{x}$ that is not above $\mathbf{b}$. Can we still find $\mathbf{a}, \mathbf{b}, \mathbf{d}, \mathbf{c}$ so that there is no such $\mathbf{x}$ ? In other words, is it possible that $\mathbf{b}$ is the least degree below $\mathbf{c}$ such that $\mathbf{a} \vee \mathbf{b} \geqslant \mathbf{d}$ ? The answer is no.

Theorem 1.23 (No-least-join theorem). Let $\mathbf{c}$ be a non-computable c.e. degree. Let $\mathbf{a}, \mathbf{b}<\mathbf{c}$ such that $\mathbf{a} \nless \mathbf{b}$ and $\mathbf{b}>\mathbf{0}$. Then there is a degree $\mathbf{x} \leqslant \mathbf{c}$ such that $\mathbf{a} \vee \mathbf{x} \geqslant \mathbf{b}$ but $\mathbf{x} \ngtr \mathbf{b}$.

Figure 4 reflects the situation of the theorem in the particular case when $\mathbf{a} \vee \mathbf{x}<\mathbf{c}$ and $\mathbf{a}$ and $\mathbf{b}$ are incomparable.

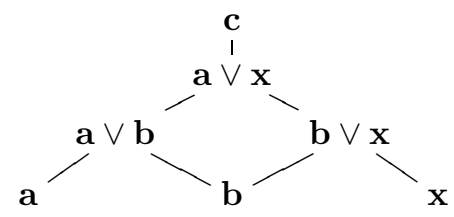

Figure 4. No least join

The restricted difference filter. The questions we have raised in this paper indicate that an important object that we need to understand better is the following.

Definition 1.24. Given Turing degrees $\mathbf{b}$ and $\mathbf{a}$, we define the difference filter as

$$
\mathbf{a} \rightarrow \mathbf{b}=\{\mathbf{x} \in \mathcal{D}: \mathbf{x} \vee \mathbf{a} \geqslant \mathbf{b}\}
$$

For the work in this paper, a more interesting notion is the restricted difference filter

$$
\mathbf{a} \rightarrow_{\mathbf{c}} \mathbf{b}=\{\mathbf{x} \leqslant \mathbf{c}: \mathbf{x} \vee \mathbf{a} \geqslant \mathbf{b}\}
$$

where $\mathbf{c}$ is a c.e. degree.

We call this set a filter just because it is closed upwards.

The following are known observations about the difference filter. Let $\mathbf{a} \not \mathbf{b}$. Then $\mathbf{a} \rightarrow \mathbf{b}$ is never an upper cone. Moreover, it always contains 1-generic degrees and minimal degrees. To see this let $\mathbf{d}=\mathbf{b} \vee \mathbf{0}^{\prime}$. It follows from Slaman and Steel's proof of the join theorem for $\mathbf{0}^{\prime}$ [SS89] that there are 1-generic degrees $\mathbf{x}$ such that 
$\mathbf{x} \vee \mathbf{a}=\mathbf{d} \geqslant \mathbf{b}$. Using the minimal complementation theorem [Lew05, we get a minimal degree $\mathbf{x}$ with $\mathbf{x} \vee \mathbf{a}=\mathbf{d} \geqslant \mathbf{b}$.

Jockusch and Slaman JS93 proved the following result (stated in a different way):

$$
\mathbf{a} \rightarrow \mathbf{b} \subseteq \mathbf{d} \rightarrow \mathbf{e} \Longleftrightarrow \text { either } \mathbf{d} \geqslant \mathbf{a} \& \mathbf{e} \leqslant \mathbf{b} \vee \mathbf{d}, \text { or } \mathbf{e} \leqslant \mathbf{d} .
$$

The behavior of the restricted difference filter is rather different. Theorem 1.11 states that there are c.e. degrees $\mathbf{0}<\mathbf{a}<\mathbf{b}<\mathbf{c}$ such that $\mathbf{a} \rightarrow_{\mathbf{c}} \mathbf{b}$ is the cone of degrees above $\mathbf{b}$, of course, restricted to $\mathcal{D}_{(\leqslant \mathbf{c})}$. On the other hand, the no-least-join theorem 1.23 states that if $\mathbf{0}<\mathbf{a}, \mathbf{b}<\mathbf{c}$, $\mathbf{c}$ is c.e. and $\mathbf{a} \mid \mathbf{b}$, then $\mathbf{a} \rightarrow_{\mathbf{c}} \mathbf{b}$ is never an upper cone.

Jockusch's and Slaman's condition does not hold anymore for the restricted filter: Theorem 1.18 provided c.e. degrees $\mathbf{a}, \mathbf{b}, \mathbf{d}, \mathbf{e}$, all incomparable and below a c.e. degree $\mathbf{c}$, such that $\mathbf{a} \rightarrow_{\mathbf{c}} \mathbf{b} \subseteq \mathbf{d} \rightarrow_{\mathbf{c}} \mathbf{e}$.

Background and notation. Our notation is standard and mostly follows [Soa87]. In particular, we use Lachlan's modifier $[s]$ to denote that an entire expression is evaluated at a stage $s$.

Many of our constructions will be organized on a tree of strategies. We assume the reader is familiar with this type of construction. See [Soa87, Chapter XIV] for background on tree constructions. Let us very quickly refresh the reader about the notation on this type of construction: Each node $\alpha$ in the tree of strategies is assigned a requirement $\mathbf{R}_{\alpha}$. These requirements have certain possible outcomes, and for each of these outcomes $o, \alpha^{\frown} o$ is another node in the tree of strategies. The idea is that each node in the tree of strategies codes the outcomes of the requirements of higher priority. The set of outcomes is linearly ordered, and this induces a lexicographic partial ordering $<_{L}$ on the whole tree, where nodes comparable under $\subseteq$ are incomparable under $<_{L}$. Nodes to the left have higher priority than nodes to the right. Also, nodes that are initial segments of other nodes have higher priority. At each stage $s$ we will pick a node $\alpha_{s}$ in the tree to be our current approximation to the true path. At this stage we will act for each requirement $\mathbf{R}_{\alpha}$ for $\alpha \subseteq \alpha_{s}$. If $\alpha \subseteq \alpha_{s}$, we say that $\alpha$ is accessible at $s$, and that $s$ is an $\alpha$-stage. The true path of a construction is the leftmost path visited infinitely often. The requirements will be satisfied by nodes lying on the true path.

A set $G$ is 1 -A-generic if for every $A$-c.e. set of strings $W$, there exists $\sigma \subset G$ such that either $\sigma \in W$, or no extension of $\sigma$ is in $W$. If $G$ is 1 - $A$-generic, then $G$ does not compute any $A$-c.e. set and if $D<_{T} A$, then $D \oplus G \ngtr_{T} A$.

A degree $\mathbf{c}$ is $G L_{2}$ if $\left(\mathbf{c} \vee \mathbf{0}^{\prime}\right)^{\prime} \geqslant \mathbf{c}^{\prime \prime}$. If $f_{1}$ is computable in $\mathbf{c} \vee \mathbf{0}^{\prime}$, and $\mathbf{c}$ is not $G L_{2}$, then there exists a c-computable function $f_{2}$ that is not dominated by $f_{1}$.

\section{Almost contiguity and the global anti-Cupping property}

In this section we prove Lemmas 1.12 and 1.15 getting Example 1.14 as a corollary. The ideas used in the proofs will be used in later sections.

2.1. An almost contiguous pair. As we said in the introduction, a degree $\mathbf{a}$ is strongly contiguous if any two sets $A, B \in \mathbf{a}$ are $w t t$-equivalent. Downey [Dow87. used these degrees to transfer properties about the structure of $w t t$-degrees to the structure of Turing degrees. We use the same idea here. However, we do not need to use strongly contiguous degrees. We just need the following lemma. 
Lemma 2.1 (Lemma 1.12). There exist c.e. sets B, A, such that $0<_{T} B<_{T} A$ and such that for every set $X$ with $B \leqslant_{T} X \leqslant_{T} A$, we have $B \leqslant_{w t t} X$.

Proof. We meet three types of requirements:

$$
\begin{aligned}
\mathbf{P}_{e}^{B, A}: & \Psi_{e}(B) \neq A, \\
\mathbf{P}_{e}^{\emptyset, B}: & \Psi_{e}(\emptyset) \neq B, \\
\mathbf{M}_{e}: & \Phi_{e}(A)=X \text { total } \wedge \Gamma_{e}(X)=B \Longrightarrow B \leqslant_{\mathrm{wtt}} X .
\end{aligned}
$$

Here $\left\langle\Psi_{e}, \Phi_{e}, \Gamma_{e}\right\rangle$ is an effective list of all triples of Turing functionals.

The requirements will be arranged on a tree of strategies as usual. To each node $\sigma$ in the tree of strategies we assign a requirement $\mathbf{R}_{\sigma}$. If $|\sigma|=3 e$, then we let $\mathbf{R}_{\sigma}=\mathbf{M}_{e}$; if $|\sigma|=3 e+1$, let $\mathbf{R}_{\sigma}=\mathbf{P}_{e}^{B, A}$; if $|\sigma|=3 e+2$, let $\mathbf{R}_{\sigma}=\mathbf{P}_{e}^{\emptyset, B}$.

We also need to make sure that $B \leqslant_{T} A$. To achieve this, we code $B$ into $A$ : whenever we enumerate any number into $B$, we also immediately enumerate it into $A$. Hence $B$ can be computed from $A$ by observing that for all $x$ and $s$, if $A_{s}\left\lceil x=A\left\lceil x\right.\right.$, then $B_{s}\lceil x=B\lceil x$.

We specify the construction by detailing, at a stage $s$, the following, for any node $\sigma$ which is accessible at stage $s$ : what $\sigma$ 's action is at the stage, whether it terminates the stage, and if it does not terminate the stage, what its outcome is. The tree of strategies is defined recursively: if $\sigma$ is a string which has already been decided to be a strategy, then for every possible outcome $o$ of $\sigma, \sigma \frown o$ is also a strategy. Our instructions will define the path $\alpha_{s}$ of nodes that are accessible at stage $s$, assuming of course that the root of the tree is always accessible. If $\sigma$ is the last node which is accessible at stage $s$, then we initialise all nodes which are weaker than $\sigma$, which means that they lose the followers that may have been previously assigned to them.

Suppose, then, that $\sigma$ is a node, accessible at stage $s$. Of course, $\sigma$ 's action depends on the requirement $\mathbf{R}_{\sigma}$ which has been assigned to $\sigma$.

2.1.1. $\mathbf{R}_{\sigma}=\mathbf{P}^{B, A}$. We use a Friedberg-Muchnik strategy [Fri57, Muc56. If $\sigma$ does not have a follower, it picks a new large one, and halts the stage. If $\sigma$ has a follower $x, \Phi_{e}(B)(x) \downarrow[s]=0$, and $x \notin A_{s}$, then $\sigma$ enumerates $x$ into $A_{s+1}$, and halts the stage. The node $\sigma$ has only one outcome, and so if $\sigma$ does not halt the stage, the unique immediate successor of $\sigma$ on the tree of strategies is next accessible.

2.1.2. $\mathbf{R}_{\sigma}=\mathbf{P}_{e}^{\emptyset, B}$. The node $\sigma$ works in exactly the same way as nodes working for $\mathbf{P}^{B, A}$, appointing a follower $x$, and then monitoring a computation $\Phi_{e}(\emptyset, x)$, and enumerating $x$ into $B_{s+1}$ when $\Phi_{e}(\emptyset, x)$ converges to 0 . It also halts the stage whenever it acts. Note that if $\sigma$ enumerates $x$ into $B_{s+1}$, it also enumerates it into $A_{s+1}$ as guaranteed above.

2.1.3. $\mathbf{R}_{\sigma}=\mathbf{M}_{e}$. The outcomes of $\sigma$ are $\infty$ and fty, ordered by $\infty<\mathrm{fty}$. We define the length of agreement $\ell_{s}(\sigma)$ to be the greatest $x$ such that $\Gamma_{e}\left(\Phi_{e}(A)\right) \uparrow x=$ $B\left\lceil x[s]\right.$. We let $\infty$ be $\sigma$ 's outcome at stage $s$ if $s$ is the least $\sigma$-stage, or if $\ell_{s}(\sigma)$ is greater than any number mentioned during the previous $\sigma^{\frown} \infty$-stage. Otherwise, we let fty be $\sigma$ 's outcome at stage $s$. 
2.1.4. Verification. The true path consists of the nodes $\sigma$ which are accessible at infinitely many stages, but no node to the left of $\sigma$ is accessible at infinitely many stages. The true path is a downward closed, $\subseteq$-linearly ordered subset of the tree of strategies. By induction, we can show that if $\sigma$ lies on the true path, then $\sigma$ has an immediate successor on the true path. If $\mathbf{R}_{\sigma}=\mathbf{M}_{e}$, then this follows from the fact that $\sigma$ never halts the stage, and that $\sigma$ has finitely many outcomes. If $\mathbf{R}_{\sigma}$ is a positive requirement $\left(\mathbf{P}_{e}^{B, A}\right.$ or $\left.\mathbf{P}_{e}^{\emptyset, B}\right)$, we need to show that $\sigma$ does not halt the stage infinitely often.

Lemma 2.2. If $\sigma$ lies on the true path, and $\mathbf{R}_{\sigma}$ is a positive requirement, then $\mathbf{R}_{\sigma}$ is met, and $\sigma$ only halts the stage finitely often.

Proof. This is argued as is usual in finite injury constructions. If $\mathbf{R}_{\sigma}=\mathbf{P}_{e}^{B, A}$, then by induction, there is a stage $s_{0}$ which is the last stage at which $\sigma$ is initialised. At the first stage $s_{1}>s_{0}$ at which $\sigma$ is accessible, $\sigma$ appoints a new follower $x$, so $x \notin A_{s_{1}}$. The follower $x$ is never cancelled. The fact that at stage $s_{1}, \sigma$ initialises all weaker nodes, together with the fact that no node stronger than $\sigma$ enumerates numbers into $A$ after stage $s_{0}$, shows that $x \in A$ if and only if $\sigma$ enumerates $x$ into $A$. It does so, at some stage $s_{2}>s_{1}$, only if $\Phi_{e}(B, x)\left[s_{2}\right]=0$, in which case $\sigma$ 's initialisation of weaker nodes at stage $s_{2}$ ensures that $B_{s_{2}} \uparrow u=B \uparrow u$, where $u$ is the use of the computation $\Phi_{e}(B, x)\left[s_{2}\right]$, and so $\Phi_{e}(B, x) \neq A(x)$. If $\Phi_{e}(B, x)=0$, then the finite use principle shows that $\sigma$ will indeed enumerate $x$ into $A$ at some stage. Otherwise, as we have ensured, $x \notin A$. Hence the requirement $\mathbf{P}_{e}^{B, A}$ is met. We have observed that $\sigma$ only ends the stage at most once after stage $s_{1}$.

A slightly simpler argument is made if $\mathbf{R}_{\sigma}=\mathbf{P}_{e}^{\emptyset, B}$. If $\sigma$ enumerates $x$ into $B$, then it also enumerates $x$ into $A$, but since the computation $\Phi_{e}(\emptyset, x)$ cannot be injured, this action does not hurt $\sigma$ 's success.

Hence the true path is infinite, every positive requirement is met, and any node on the true path is initialised finitely many times. We turn to show that every requirement $\mathbf{M}_{e}$ is met. Let $\sigma$ be a node on the true path such that $\mathbf{R}_{\sigma}=\mathbf{M}_{e}$. We suppose that $X=\Phi_{e}(A)$ is total, and that $B=\Gamma_{e}(X)$; we show that $B \leqslant_{\mathrm{wtt}} X$. The finite use principle shows that there are infinitely many $\sigma \frown \infty$-stages (also known as " $\sigma$-expansionary" stages), and so, as $\infty<$ fty, the node $\sigma \frown \infty$ lies on the true path. Let $s_{0}$ be the last stage at which $\sigma$, and hence $\sigma \frown \infty$, are initialised.

For a $\sigma \frown \infty$-stage $s$, let $u(s)$ be the $A$-use of the computation $\Gamma_{e}\left(\Phi_{e}(A)\right) \uparrow \ell(\sigma)=$ $B \nmid \ell(\sigma)[s]$, and let $X_{s}=\Phi_{e}(A)$; so $X_{s}$ is a finite string, and $\Gamma_{e}\left(X_{s}\right) \supseteq B_{s} \uparrow \ell_{s}(\sigma)$. For $n>0$, let $s_{n}$ be the least $\sigma \frown \infty$-stage $s>s_{0}$ such that $\ell_{s}(\sigma)>n$; the map $n \mapsto s_{n}$ is computable, and so the map $n \mapsto v(n)=\left|X_{s_{n}}\right|$ is computable.

Lemma 2.3. If $t>s_{n}$ is a $\sigma \frown \infty$-stage and $X_{t} \uparrow v(n)$ is $X$-correct, then $B(n)=$ $B_{t}(n)$.

Lemma 2.3 gives a weak truth-table procedure for computing $B$ from $X$, as a stage $t$ as in the lemma can be effectively obtained from $X$, with use $v(n)$.

To prove Lemma2.3 fix $n$ and $t>s_{n}$ as in the lemma, and suppose that $n \notin B_{t}$; we need to show that $n \notin B$. As all elements of $B$ are followers for nodes $\tau$ with $\mathbf{R}_{\tau}=\mathbf{P}_{i}^{\emptyset, B}$ for some $i$, we suppose that there is a node, $\tau$, such that $\mathbf{R}_{\tau}$ is a positive requirement, and such that $n$ is a follower for $\tau$ at a stage $t^{\prime} \geqslant t$; we show that $\tau$ does not enumerate $n$ into $B$ after stage $t$. 
We may assume that $\tau$ is not stronger than $\sigma$, for as $t>s_{0}$, nodes that are stronger than $\sigma$ do not act after stage $t$. Let $s$ be the stage at which $\tau$ appointed $n$ as a follower.

Claim 2.4. $\tau \supseteq \sigma^{\frown} \infty$, so $s$ is a $\sigma \frown \infty$-stage; it is, in fact, the greatest $\sigma \frown \infty$-stage before stage $s_{n}$.

Proof. At stage $s, n$ is large. Since $n<s_{n}$, we have $s<s_{n}$. At stage $s_{n}$, all nodes that lie to the right of $\sigma^{\frown} \infty$ are initialised, and so if $\tau$ lies to the right of $\sigma^{\frown} \infty$, then any follower it may have at a stage $r \geqslant t$ must be appointed at a stage after stage $s_{n}$, and not before it. Since we assume that $\tau$ is not stronger than $\sigma$, we have $\tau \supseteq \sigma \frown \infty$.

It follows that $s$ is a $\sigma \frown \infty$-stage. Since $n$ is mentioned at stage $s$, at the next $\sigma \frown \infty$-stage $s^{\prime}$ we have $\ell_{s^{\prime}}(\sigma)>n$, and so $s^{\prime} \geqslant s_{n} ;$ so $s^{\prime}=s_{n}$.

Claim 2.5. $A_{t}\left\lceil u\left(s_{n}\right)=A_{s}\left\lceil u\left(s_{n}\right)\right.\right.$.

Proof. If not, then some node $\rho$ enumerates a follower $x<u\left(s_{n}\right)$ into $A$ at some stage $r \in[s, t)$. The argument for Claim 2.4 shows that $\rho \supseteq \sigma^{\frown} \infty$. Since $n$ is a follower for $\tau$ at stage $r$ and is not cancelled at that stage, $\rho$ must be weaker than $\tau$. This implies that $\rho$ is initialised at stage $s$, so $\rho$ appoints $x$ at a stage $w \geqslant s_{n}$. As new followers are chosen large, this implies that $x>u\left(s_{n}\right)$, for a contradiction.

It follows that $X_{s_{n}}$ is an initial segment of $X_{t}$, and so, by the choice of $t$, that $X_{s_{n}}$ is an initial segment of $X$. It follows that $B_{s}$ agrees with $B$ up to $\ell_{s}(\sigma)$, and so that $B(n)=B_{s}(n)=0$, as required.

\subsection{The global anti-cupping property.}

Lemma 2.6 (Lemma 1.15). There exist Turing-incomparable disjoint c.e. sets $A_{0}$ and $A_{1}$ such that for every set $X$,

$$
X \oplus A_{0} \geqslant_{w t t} B \quad \& \quad X \oplus A_{1} \geqslant_{w t t} B \Longrightarrow X \geqslant_{T} B
$$

where $B=A_{0} \cup A_{1}$.

Proof. We meet three types of requirements:

$$
\begin{aligned}
\mathbf{P}_{e}^{A_{0}, A_{1}}: & \Psi_{e}\left(A_{0}\right) \neq A_{1}, \\
\mathbf{P}_{e}^{A_{1}, A_{0}}: & \Psi_{e}\left(A_{1}\right) \neq A_{0}, \\
\mathbf{N}_{e}: & \hat{\Phi}_{e}\left(A_{0} \oplus X\right)=\hat{\Phi}_{e}\left(A_{1} \oplus X\right)=B \Longrightarrow X \geqslant_{T} B,
\end{aligned}
$$

where we use $\hat{\Phi}_{e}$ to denote a weak truth-table functional with use $\varphi_{e}$. We agree that if $x \notin \operatorname{dom} \varphi_{e}$ then $\hat{\Phi}_{e}(X, x) \uparrow$ for any $X$, and that for all $s, \operatorname{dom} \varphi_{e, s}$ is an initial segment of $\omega$.

We again use a tree of strategies for the construction; the requirements are effectively ordered in order-type $\omega$; the requirement in place $e$ is assigned to all nodes of level $e$ of the tree. We again fully describe the tree and the construction by describing how to act when a node $\sigma$ is accessible at a stage $s$; at the end of a stage, we again initialise all nodes weaker than the last accessible node.

2.2.1. $\mathbf{R}_{\sigma}=\mathbf{P}_{e}^{A_{i}, A_{1-i}}$. The node $\sigma$ acts exactly as the nodes working for positive requirements did in the construction proving Lemma 1.12, adopting the FriedbergMuchnik strategy of appointing followers and initialising when necessary. Such nodes, again, have a single outcome. 
2.2.2. $\mathbf{R}_{\sigma}=\mathbf{N}_{e}$. The node $\sigma$ guesses whether $\varphi_{e}$ is total or not. It has two outcomes, $\infty<$ fty. If $s$ is the least $\sigma$-stage, or if $\varphi_{e, s}(x) \downarrow$, where $x$ is the greatest number mentioned in the previous $\sigma \frown \infty$-stage, then $\infty$ is $\sigma$ 's outcome at stage $s$; otherwise, fty is $\sigma$ 's outcome at stage $s$.

2.2.3. Verification. The verification of the fairness of the construction (the fact that on the true path, every node is initialised only finitely often), and that every positive requirement is met, is precisely as in the verification of the proof of Lemma 1.12. The sets $A_{0}$ and $A_{1}$ are disjoint since each number can be a follower for at most one node. We show that each requirement $\mathbf{N}_{e}$ is met; let $\sigma$ be the node on the true path such that $\mathbf{R}_{\sigma}=\mathbf{N}_{e}$. Let $X \in 2^{\omega}$, and suppose that $\varphi_{e}$ is total and that $\hat{\Phi}_{e}\left(A_{0} \oplus X\right)=\hat{\Phi}_{e}\left(A_{1} \oplus X\right)=B$. We show that $X \geqslant_{T} B$. Certainly $\sigma \frown \infty$ lies on the true path. Let $s_{0}$ be the last stage at which $\sigma$ is initialised.

With oracle $X$, we compute $B\lceil n$ by recursion on $n$. Suppose that $B \uparrow n$ has been computed; we can find a $\sigma \frown \infty$-stage $s_{n}>s_{0}, n$ such that $B \uparrow n=B_{s_{n}} \uparrow n$. The arguments for Lemma1.12 show that unless at stage $s_{n}, n$ is a follower for some node extending $\sigma^{\frown} \infty$, then $B(n)=B_{s_{n}}(n)$. Suppose then that $\tau \supseteq \sigma^{\frown} \infty$ is a node such that $\mathbf{R}_{\tau}$ is positive and that at stage $s_{n}, n$ is a follower for $\tau$. Without loss of generality, suppose that $\mathbf{R}_{\tau}=\mathbf{P}_{i}^{A_{0}, A_{1}}$, so $n$ is targeted for $A_{1}$. Let $s$ be the stage at which $\tau$ appointed $n$ as a follower. Again, the previous arguments show that $s$ is the greatest $\sigma^{\frown} \infty$-stage before stage $s_{n}$, and that by virtue of $\tau$ 's initialising action at stage $s$, no follower $x$ for any node at stage $s_{n}$ lies in the interval $\left(n, \varphi_{e}(n)\right]$. Now the fact that $B_{s_{n}} \uparrow n=B \uparrow n$ implies that $B_{s_{n}}(x)=B(x)$ for all $x \leqslant \varphi_{e}(n)$, except possibly for $x=n$, and since $n$ is targeted for $A_{1}$, we know that $A_{0}(n)=0$, and so $A_{0, s_{n}}\left\lceil\varphi_{e}(n)=A_{0}\left\lceil\varphi_{e}(n)\right.\right.$. Given $A_{0}\left\lceil\varphi_{e}(n)\right.$ we can now compute, with oracle $X$, the desired value $B(n)=\hat{\Phi}_{e}\left(A_{0} \oplus X, n\right)$.

Note that in the last argument we only needed $X \uparrow \varphi_{e}(n)$, so in fact we get $B \leqslant w t t X$.

\section{The GENERALIZED ANTI-CUPPING CONDITION}

This section is dedicated to proving Theorem 1.17, the necessity of the anticupping condition for membership in $\mathbb{E}^{\text {c.e. }}$.

3.1. Upper-semi-lattice embeddings into the c.e. degrees. We start by describing the standard method of embedding a usl in the c.e. degrees. We include the proof here because we will use a modification of it later, and so we describe it in a way that is compatible with this modification.

Lemma 3.1 (Friedberg, Muchnik [Fri57, Muc56]). Every finite usl can be embedded into the c.e. degrees.

Proof. Let $\mathcal{P}$ be a finite usl. We build c.e. sets $A^{\mathbf{a}}$ for each $\mathbf{a} \in \mathcal{P}$ such that the map $\mathbf{a} \mapsto \operatorname{deg}_{T}\left(A^{\mathbf{a}}\right)$ is a usl-embedding.

Let $\mathcal{P}$ be a usl. A subset $F$ of $\mathcal{P}$ is said to be a filter if it is closed upwards and whenever $\mathbf{a} \vee \mathbf{b} \in F$, at least one of $\mathbf{a}$ or $\mathbf{b}$ is in $F$.

When $\mathbf{a} \leqslant \mathbf{b}$, we need to get $A^{\mathbf{a}} \leqslant_{T} A^{\mathbf{b}}$. For this purpose, we impose the condition that whenever we enumerate some $x$ into $A^{\mathbf{a}}$ we also enumerate it immediately into $A^{\mathbf{b}}$; we saw in the previous section that this indeed implies that $A^{\mathbf{a}} \leqslant_{T} A^{\mathbf{b}}$. 
When $\mathbf{a} \vee \mathbf{b} \geqslant \mathbf{c}$ we want to have that $A^{\mathbf{a}} \oplus A^{\mathbf{b}} \geqslant_{T} A^{\mathbf{c}}$. For this purpose, we impose the condition that whenever we enumerate some $x$ into $A^{\mathbf{c}}$, we also immediately enumerate it into either $A^{\mathbf{a}}$ or $A^{\mathbf{b}}$.

We summarize these two conditions into one, recalling that at each stage, at most one number is enumerated into sets:

(F1) If a number $x$ is enumerated into a set at stage $s$, then the collection of a such that $x$ is enumerated into $A^{\text {a }}$ at stage $s$ forms a filter of $\mathcal{P}$.

In fact, the only filters we use will be the complements of principal ideals: for $\mathbf{d} \in \mathcal{P}$,

$$
F_{\mathbf{d}}=\{\mathbf{z} \in \mathcal{P}: \mathbf{z} \not \mathbf{d}\} .
$$

The next thing we need is that whenever $\mathbf{d} \ngtr \mathbf{e}, A^{\mathbf{d}} \Varangle_{T} A^{\mathbf{e}}$. Note that since $\mathcal{P}$ is an upper-semi-lattice, $\mathbf{e} \vee \mathbf{d} \in \mathcal{P}$ and it is enough to get $A^{\mathbf{d}} \ngtr_{T} A^{\mathbf{e} \vee \mathbf{d}}$. Hence, it is enough to satisfy the following requirements, for each $\mathbf{d}, \mathbf{e} \in \mathcal{P}$ with $\mathbf{d}<\mathbf{e}$, and each $e \in \omega$ :

$$
\mathbf{P}_{e}^{\mathbf{d}, \mathbf{e}}: \quad \Psi_{e}(D) \neq E .
$$

As in the previous section, these requirements are effectively ordered in ordertype $\omega$. Since all requirements are finitary, we have no need for a tree of strategies; we carry out a finite injury argument. At each stage, we consider each requirement in turn, and either pass to the next requirement, or act for the requirement under consideration, halt the stage, and initialise all weaker requirements.

3.1.1. The requirement $\mathbf{P}_{e}^{\mathbf{d}, \mathbf{e}}$. Our action for this requirement is identical to our action for positive requirements in the previous section; the coding we need to undertake so as to meet condition (F1) is similar to our promise, in the proof of Lemma 1.12, to enumerate every follower targeted for $B$ into the set $A$ as well. The requirement $\mathbf{P}_{e}^{\mathbf{d}, \mathbf{e}}$ will appoint a large follower $x$, wait for $\Phi_{e}\left(A^{\mathbf{d}}, x\right) \downarrow=0$, and then enumerate $x$ into $A^{\mathbf{a}}$ for all $\mathbf{a} \in F_{\mathbf{d}}$, that is, into every a not below $\mathbf{d}$. Note that $\mathbf{d}<\mathbf{e}$ implies that $\mathbf{e} \in F_{\mathbf{d}}$, so $x$ is enumerated into $A^{\mathbf{e}}$.

3.1.2. Verification. Again this is identical to the verification for Lemma 1.12, The only point is that we obeyed the condition (F1), thus obtaining the reductions required, while making sure not to injure the computations $\Phi_{e}\left(A^{\mathbf{d}}, x\right)=0$ against which we diagonalised, and so we managed to preserve them.

3.2. A negative condition. In this subsection we put together the constructions of the previous three subsections and prove Theorem 1.17. So, let us consider a pair of usls $\mathcal{P} \subset \mathcal{Q}$ such that $(\mathcal{P}, \mathcal{Q}) \forall \neq$ ACC. There has to be some $\mathbf{x} \in \mathcal{Q} \backslash \mathcal{P}$ and $\mathbf{a} \mathbf{b} \in \mathcal{P}, \mathbf{b} \notin \mathbf{x}$ such that if $\mathbf{a}_{1}, \ldots, \mathbf{a}_{k}$ are the $\mathcal{P}$-minimal elements below $\mathbf{b}$ such that $\mathbf{a}_{i} \vee \mathbf{x} \geqslant \mathbf{b}$, then for every $\mathbf{c} \in \mathcal{P}$ with $\mathbf{c} \ngtr \mathbf{b}$, there exists some $i$ such that $\mathbf{a}_{i} \vee \mathbf{c} \ngtr \mathbf{b}$.

We want to construct an embedding $\mathbf{a} \mapsto \operatorname{deg}_{T}\left(A^{\mathbf{a}}\right)$ of $\mathcal{P}$ into the c.e. degrees which has no extension to an embedding of $\mathcal{P}[\mathbf{x}]$ into $\mathcal{D}\left(\leqslant \operatorname{deg}_{T}\left(A^{\mathbf{1}}\right)\right)$.

We use $A$ to denote $A^{\mathbf{1}}$ and $B$ to denote $A^{\mathbf{b}}$. To ensure that this embedding of $\mathcal{P}$ has no extension to an embedding of $\mathcal{P}[\mathbf{x}]$ into $\mathcal{D}\left(\leqslant \operatorname{deg}_{T}(A)\right)$, we construct the embedding so that there is no $X \leqslant_{T} A$ such that $X ¥_{T} B$ and for every $i=1, \ldots, k, X \oplus A^{\mathbf{a}_{i}} \geqslant_{T} B$. For this purpose, we build $B$ and $A$ such that for every $Y$, if $B \leqslant_{T} Y \leqslant_{T} A$, we have $B \leqslant_{w t t} Y$, and such that for every $X$, if for every $i=1, \ldots, k, X \oplus A^{\mathbf{a}_{i}} \geqslant_{w t t} B$, then $X \geqslant_{T} B$. 
For each $\mathbf{d}, \mathbf{e} \in \mathcal{P}$ with $\mathbf{d}<\mathbf{e}$, we meet the requirements:

$$
\begin{aligned}
\mathbf{P}_{e}^{\mathbf{d}, \mathbf{e}}: & \Psi_{e}\left(A^{\mathbf{d}}\right) \neq A^{\mathbf{e}}, \\
\mathbf{M}_{e}: & \Phi_{e}(A) \text { total } \wedge \Gamma_{e}\left(\Phi_{e}(A)\right)=B \Longrightarrow B \leqslant_{\mathrm{wtt}} \Phi_{e}(A), \\
\mathbf{N}_{e}: & \forall i=1, \ldots, k \hat{\Phi}_{e}\left(A^{\mathbf{a}_{i}} \oplus X\right)=B \Longrightarrow X \geqslant_{T} B .
\end{aligned}
$$

Also, if $\mathbf{d}, \mathbf{e} \in \mathcal{P}$ with $\mathbf{d} \leqslant \mathbf{e}$, we need to get that $A^{\mathbf{d}} \leqslant_{T} A^{\mathbf{e}}$, and for any $\mathbf{d}$ and $\mathbf{e}$, we need $A^{\mathbf{d} \cup \mathbf{e}} \leqslant_{T} A^{\mathbf{e}} \oplus A^{\mathbf{d}}$. We will obtain these by obeying a condition similar to (F1). We cannot use condition (F1) verbatim, because this would injure meeting the requirements $\mathbf{N}_{e}$. What made the argument for Lemma 1.15 work was the fact that if $x$ entered $B=A_{0} \cup A_{1}$, then it entered exactly one of $A_{0}$ and $A_{1}$, and not both, thus preserving a $\hat{\Phi}_{e}$-computation, or in a sense, "forcing a change in $X$ ". In the current construction, we need to avoid the situation that a number enters $B$, but at the same time enters every $A^{\mathbf{a}_{i}}$ for $i=1, \ldots, k$. Call a filter $F$ of $\mathcal{P}$ amenable if $\mathbf{b} \notin F$, or if $\mathbf{a}_{i} \notin F$ for some $i \leqslant k$. Now we will not be able to require that condition (F1) holds, strengthened by the requirement that the promised filter is amenable: for $\mathbf{d}<\mathbf{e}$, there may not always be an amenable filter $F$ containing e but not $\mathbf{d}$; this is the case if for all $i \leqslant k, \mathbf{a}_{i} \vee \mathbf{d} \geqslant \mathbf{e}$. We relax the condition as follows:

(F2) Suppose that numbers are enumerated into sets at stage $s$. There are two possibilities:

(a) Exactly one number $x$ is enumerated into sets at stage $s$. The collection of $\mathbf{a} \in \mathcal{P}$ such that $x$ enters $A^{\mathbf{a}}$ at stage $s$ is an amenable filter of $\mathcal{P}$.

(b) Exactly two numbers, $x_{0}<x_{1}$, are enumerated into sets at stage $s$. For each $i=0,1$, the collection $F_{i}$ of $\mathbf{a} \in \mathcal{P}$ such that $x_{i}$ enters $A^{\mathbf{a}}$ at stage $s$ is a filter of $\mathcal{P}$, with $F_{0}$ amenable, $F_{0} \subset F_{1}$, and $\mathbf{b} \in F_{0}$.

Before we specify the construction, we first note that if our construction meets the condition ( $(\overline{\mathrm{F} 2})$, then it is still the case that for any stage $s$ and any number $x$, the collection of $\mathbf{a} \in \mathcal{P}$ such that $x$ enters $A^{\mathbf{a}}$ at stage $s$ is a filter of $\mathcal{P}$. It follows that the positive relations are met: if $\mathbf{d}<\mathbf{e}$, then $A^{\mathbf{d}} \leqslant_{T} A^{\mathbf{e}}$, and for any $\mathbf{d}$ and $\mathbf{e}$, $A^{\mathbf{d} \vee \mathbf{e}} \leqslant_{T} A^{\mathbf{d}} \oplus A^{\mathbf{e}}$.

We also verify that every Friedberg-Muchnik type requirement can find filters that meet condition ( $\underline{\mathrm{F} 2})$ :

Lemma 3.2. Suppose that $\mathbf{d}$ and $\mathbf{e}$ are elements of $\mathcal{P}$, and that $\mathbf{d}<\mathbf{e}$. Then either:

(1) there is an amenable filter $F$ of $\mathcal{P}$ such that $\mathbf{e} \in F$ and $\mathbf{d} \notin F$; or

(2) there are non-empty filters $F_{0} \subset F_{1}$ of $\mathcal{P}$ such that $F_{0}$ is amenable, $\mathbf{b} \in F_{0}$, $\mathbf{e} \in F_{1}$, and $\mathbf{d} \notin F_{1}$.

Proof. If there is some $i \leqslant k$ such that $\mathbf{a}_{i} \vee \mathbf{d} \ngtr \mathbf{e}$, then $F=F_{\mathbf{a}_{i} \vee \mathbf{d}}$ is amenable, contains e, and not $\mathbf{d}$. Otherwise, $\mathbf{d} \vee \mathbf{b} \geqslant \mathbf{e}$, and so $\mathbf{d} \nless \mathbf{b}$; so since $(\mathcal{P}, \mathcal{P}[\mathbf{x}]) \not$ ACC, there is some $i \leqslant k$ such that $\mathbf{d} \vee \mathbf{a}_{i} \ngtr \mathbf{b}$. We then let $F_{0}=F_{\mathbf{d} \vee \mathbf{a}_{i}}$, and $F_{1}=F_{\mathbf{d}}$.

We are now ready to describe the construction. As before, we describe the tree of strategies by describing, for a node $\sigma$ which is accessible at stage $s$, what action we take for $\sigma$, depending on the nature of $\mathbf{R}_{\sigma}$, and if $\sigma$ does not halt the stage, which outcome of $\sigma$ is taken at stage $s$. As before, if $\sigma$ halts the stage, then all nodes weaker than $\sigma$ are initialised. 
3.2.1. $\mathbf{R}_{\sigma}=\mathbf{M}_{e}$. The node $\sigma$ acts exactly like its counterparts in the construction for Lemma 1.12 (Subsection 2.1.3). It monitors the length of agreement $\ell_{s}(\sigma)$ between $\Gamma_{e}\left(\Phi_{e}(A)\right)$ and $B$; the outcome is $\infty$ if this is greater than the largest number mentioned in the previous $\sigma^{\frown} \infty$-stage, and fty otherwise.

3.2.2. $\mathbf{R}_{\sigma}=\mathbf{N}_{e}$. The node $\sigma$ acts exactly like its counterparts in the construction for Lemma 1.15](Subsection 2.2.2). It monitors the length of convergence of $\varphi_{e}$; the outcome is $\infty$ if this is greater than the largest number mentioned in the previous $\sigma \frown \infty$-stage, and fty otherwise.

3.2.3. $\mathbf{R}_{\sigma}=\mathbf{P}_{e}^{\mathbf{d}, \mathbf{e}}$. If there is an amenable filter $F$ of $\mathcal{P}$ such that $\mathbf{e} \in F$ and $\mathbf{d} \notin F$, then the node $\sigma$ chooses such a filter $F$ and acts as the requirement acted in the construction for Lemma 3.1 appointing a follower $x$, waiting for $\Phi_{e}\left(A^{\mathbf{d}}, x\right) \downarrow=0$, and then enumerating $x$ into $A^{\mathbf{a}}$ for all $\mathbf{a} \in F$.

If there is no such filter $F$, the node $\sigma$ chooses filters $F_{0}$ and $F_{1}$ as guaranteed by Lemma 3.2. At the first visit to $\sigma$ (after it is initialised), $\sigma$ appoints a fresh agitator $x_{0}$, and halts the stage. At the next visit to $\sigma$, it appoints a fresh follower $x_{1}$, and again halts the stage. At later visits, we wait for $\Phi_{e}\left(A^{\mathrm{d}}, x_{1}\right) \downarrow=0$. If this occurs, $\sigma$ enumerates $x_{0}$ into $A^{\mathbf{a}}$ for all $\mathbf{a} \in F_{0}$, and $x_{1}$ into $A^{\mathbf{a}}$ for all $\mathbf{a} \in F_{1}$, and halts the stage.

3.2.4. Verification. The fairness of the construction, the infinity of the true path, and the success of the positive requirements are argued as in the previous constructions. If $\sigma$ is on the true path and $\mathbf{R}_{\sigma}=\mathbf{P}_{e}^{\mathbf{d}, \mathbf{e}}$, then $\sigma$ eventually appoints a follower $x$ and possibly also an agitator $y<x$, and neither are later cancelled. The only point to mention is that if $\Phi_{e}\left(A^{\mathbf{d}}, x\right) \downarrow=0$ at some later $\sigma$-stage $s$, then $\sigma$ enumerates $x$ into $A^{\mathrm{e}}$ at stage $s$, and does not enumerate any number (either $x$ or the associated agitator if it exists) into $A^{\mathbf{d}}$, and so preserves the computation and meets its requirement.

The verification that the requirements $\mathbf{M}_{e}$ are met is identical to the verification of Lemma 1.12. To prove Lemma 2.3 again, we let $\tau$ be a node such that at some stage $r \geqslant t, n$ is either a follower or an agitator for $\tau$. It does not matter which of them is the case; the verification proceeds verbatim in either case.

The use of amenable filters shows that the $\mathbf{N}$-requirements are met. Suppose that $\sigma$ lies on the true path and that $\mathbf{R}_{\sigma}=\mathbf{N}_{e}$. Let $X \in 2^{\omega}$; suppose that $\varphi_{e}$ is total, and that for all $i \leqslant k, \hat{\Phi}_{e}\left(A^{\mathbf{a}_{i}} \oplus X\right)=B$; we show that $X \geqslant_{T} B$. Again, with oracle $X$, we compute $B \uparrow n$ by induction on $n$. We let $s_{0}$ be the last stage at which $\sigma$ is initialised. We know that there are infinitely many $\sigma \frown \infty$-stages. Once $B \nmid n$ has been determined, we again find a $\sigma \frown \infty$-stage $s_{n}>s_{0}, n$ such that $B\left\lceil n=B_{s_{n}} \uparrow n\right.$. Suppose that $\tau \supseteq \sigma \frown \infty$ is a node with $\mathbf{R}_{\sigma}=\mathbf{P}_{i}^{\mathbf{d}, \mathbf{e}}$ for some $i, \mathbf{d}$, and $\mathbf{e}$, and that at stage $s_{n}, n$ is either a follower or an agitator for $\tau$. (If there is no such node $\tau$, then certainly $B(n)=B_{s_{n}}(n)$.)

Now we claim that we may assume that there is an amenable filter $F$ of $\mathcal{P}$ such that $\tau$ targets $n$ to enter $A^{\mathbf{a}}$ if and only if $\mathbf{a} \in F$ and does not enumerate any smaller number into any set after stage $s_{n}$. This holds if there is an amenable filter $F$ such that $\mathbf{e} \in F$ and $\mathbf{d} \notin F$ (in which case $n$ is the follower for $\tau$, and $\tau$ has no agitator), and also if $n$ is the agitator for $\sigma$ (in which case $F=F_{0}$ as chosen by $\tau$ ). Otherwise, $n$ is the follower for $\tau$, and $\tau$ has an agitator $y<n$ targeted for all $A^{\text {a }}$ for $\mathbf{a} \in F_{0}$, and we required that $\mathbf{b} \in F_{0}$; so $\tau$ targets $y$ for $B$. Since $n \notin B_{s_{n}}$, we 
have $y \notin B_{s_{n}}$, and so the inductive assumption implies that $y \notin B$; so $\tau$ does not enumerate either $y$ or $n$ into $B$, and so $B(n)=B_{s_{n}}(n)=0$.

The argument of the verifications of Lemmas 1.12 and 1.15 shows that the stage $s$ at which $\tau$ appointed $n$ is the greatest $\sigma^{\frown} \infty$-stage before stage $s_{n}$. This means that if $n$ is the agitator for $\tau$, then the associated follower $x$ for $\tau$ is appointed not before stage $s_{n}$, and so that $x>\varphi_{e}(n)$.

With the amenable filter $F$, we can proceed with following the verification of Lemma 1.15. If $\mathbf{b} \notin F$, then $\tau$ does not target $n$ for $B$, and so $B(n)=B_{s_{n}}(n)=0$. If $\mathbf{b} \in F$, then there is some $i \leqslant k$ such that $\mathbf{a}_{i} \notin F$; so $\tau$ does not target $n$ for $A^{\mathbf{a}_{i}}$. If $\tau$ targets its follower $x$ for $A^{\mathbf{a}_{i}}$, we know that $x>\varphi_{e}(n)$. Hence the previous argument shows that $A_{s_{n}}^{\mathbf{a}_{i}} \uparrow \varphi_{e}(n)=A^{\mathbf{a}_{i}} \uparrow \varphi_{e}(n)$, and so that $X$ can compute $B(n)=\hat{\Phi}\left(A^{\mathbf{a}_{i}} \oplus X, n\right)$.

\section{JOINS THAT IMPLY OTHER JOINS}

In this section we prove the following theorem.

Theorem 4.1 (Theorem 1.18). There exist c.e. sets $A, B, C, D$ and $E$ such that $A, B, D$ and $E$ are all Turing reducible to $C$ and pairwise incomparable, and such that any $\Delta_{2}^{0}$ set $X$ which is computable in $C$ and joins $A$ above $B$ also joins $D$ above $E$.

We meet two types of requirements:

$$
\begin{aligned}
\mathbf{P}_{e}^{Z_{e}, Y_{e}}: & Y_{e} \neq \Psi_{e}\left(Z_{e}\right), \\
\mathbf{M}_{e}: & \left(\Psi_{e}(C)=X \text { and } \Phi_{e}(A \oplus X)=B\right) \Rightarrow X \oplus D \geqslant_{T} E .
\end{aligned}
$$

Here $\left\langle\Psi_{e}, Y_{e}, Z_{e}\right\rangle$ is an effective list of all triples consisting of a Turing functional and of a pair of distinct sets from $\{A, B, D, E\}$.

In this construction, in order to meet the requirements $\mathbf{M}_{e}$, we will ensure that whenever a number (a follower) enters either $A$ or $E$, an even smaller number (an agitator) immediately enters either $B$ or $D$. We can thus let $C=B \oplus D$; our action will ensure that $A, E \leqslant_{T} C$. The reason for this action is the following. Suppose that $X=\Psi_{e}(C)$ is total and that $\Phi_{e}(A \oplus X)=B$; we want to compute $E$ with oracle $X \oplus D$. Suppose that we have already recursively computed $E\lceil n$ and wonder whether $n$ will later enter $E$. Such an entry will force an agitator to enter either $D$ or $B$, but with the oracle $D$, we can find a stage after which the first option is impossible. Now with $X$ fixed, the equation $\Phi_{e}(A \oplus X)=B$ means that any agitator entering $B$ must cause an $A$-change, necessarily by some follower entering $A$. This, in turn, necessitates an even smaller agitator to enter $B$, and so perpetrates an infinite descending sequence of natural numbers. Note that ideally, all agitators would enter $B$, but of course this is impossible for requirements $\mathbf{P}_{e}^{B, Y}$; this is why we need the set $D$. We mention that this argument is similar to the Slaman-Steel argument SS89] producing a c.e. degree which fails the join property.

As before, we specify the construction by observing the action of a node $\sigma$, accessible at stage $s$.

4.0.5. $\mathbf{R}_{\sigma}=\mathbf{P}_{e}^{Z, Y}$. The node $\sigma$ acts similarly to the previous construction (for Theorem 1.17). When first visited after initialisation, $\sigma$ picks an agitator $y$, and halts the stage. The agitator is targeted for $B$, unless $Z=B$, in which case it is targeted for $D$. At the next stage at which $\sigma$ is visited, $\sigma$ picks a follower $x$, targeted for $Y$, and halts the stage. We then wait for a $\sigma$-stage $s$ at which we 
observe that $\Psi_{e}(Z, x) \downarrow=0$, in which case we enumerate $x$ into $Y$, the agitator into the set for which it is targeted, and halt the stage.

4.0.6. $\mathbf{R}_{\sigma}=\mathbf{M}_{e}$. The node $\sigma$ works in a similar way to the nodes working for requirements $\mathbf{M}$ and $\mathbf{N}$ of the previous constructions. It monitors the length of agreement $\ell_{s}(\sigma)$ between $\Phi_{e}\left(A, \Psi_{e}(C)\right)$ and $B$, and picks the outcome $\infty$ if this is greater than the greatest number mentioned in the previous $\sigma \frown \infty$-stage; otherwise the outcome is fty.

4.0.7. Verification. Again, the fairness of the construction, the infinity of the true path, and the success of all positive requirements is straightforward and identical to previous constructions; note that all of these reductions are weak truth-table reductions. We verify that each requirement $\mathbf{M}_{e}$ is met. Fix $e$, and let $\sigma$ be the node on the true path such that $\mathbf{R}_{\sigma}=\mathbf{M}_{e}$. Suppose that $X=\Psi_{e}(C)$ is total, and that $\Phi_{e}(A \oplus X)=B$. There are infinitely many $\sigma \frown \infty$-stages. Let $s_{0}$ be the last stage at which $\sigma$ is initialised. By recursion on $n$, with oracle $X \oplus D$ we compute $E \uparrow n$. Given $E \uparrow n$, we find a $\sigma \frown \infty$-stage $s>s_{0}, n$ at which $E_{s} \uparrow n=E \uparrow n$, $D_{s} \uparrow n=D \uparrow n$, and $\ell_{s}(\sigma)>n$, and also such that $\Psi_{e}(C)[s] \uparrow u=X\lceil u$, where $u$ is the use of computing $B_{s} \uparrow n$ from $A_{s} \oplus \Psi_{e}(C)[s]$ via $\Phi_{e}$ (and we assume that $u \geqslant n)$. We show that $E(n)=E_{s}(n)$.

Suppose, towards a contradiction, that $n$ enters $E$ after stage $s$. Let $m$ be the smallest follower which is enumerated into any set after stage $s$; so $m \leqslant n$. Let $\tau$ be the node which enumerates $m$. Let $t$ be the stage at which $\tau$ picked $m$ as a follower; and let $r$ be the stage at which $\tau$ picked the associated agitator, $y$. The fact that $m$ is not cancelled at stage $s$ implies that $\tau$ does not lie to the right of $\sigma \frown \infty$; as $s>s_{0}$, we conclude that $\tau \supseteq \sigma \frown \infty$. As $s>n \geqslant m$, we conclude that $t<s$.

We argue that $y$ cannot be enumerated into either $D$ or $B$, contradicting the fact that $m$ is enumerated into the set for which it is targeted. The fact that $D_{s} \uparrow n=D \uparrow n$ and $y<m \leqslant n$ implies that $y \notin D$. It remains to show that $y \notin B$.

As both $r$ and $t$ are $\sigma \frown \infty$-stages, we know that $\ell_{t}(\sigma)>y$. Let $v$ be the $A \oplus \Psi_{e}(C)$ use of computing $B(y)$ via $\Phi_{e}$ at stage $t$, and let $w \geqslant v$ be the $C$-use of computing $\Psi_{e}(C)[t] \uparrow v$. As $m$ is chosen large at stage $t$, we have $m>w$. The follower $m$ is not cancelled between stages $t$ and $s$. At stage $t$, no number is enumerated into any set, and all nodes weaker than $\tau$ are initialised. We conclude that $C_{t} \uparrow w=C_{s} \uparrow w$. This implies that $\Psi_{e}(C)[s]\left\lceil v=\Psi_{e}(C)[t]\left\lceil v\right.\right.$, and also that $A_{s}\left\lceil v=A_{t}\lceil v\right.$ (as $A$ is reducible to $C$ with constant use). Hence $v \leqslant u$. As $m$ is the smallest follower to enter any set after stage $s$, and only followers enter $A$, we have $A_{s} \uparrow m=A \uparrow m$. As $m>w \geqslant v$, we have $A_{s} \uparrow v=A \uparrow v$. Since $\Psi_{e}(C)[s] \uparrow u=X \uparrow u$, we have $\Psi_{e}(C)[s] \uparrow v=X \uparrow v$. It follows that

$$
B(y)=\Phi_{e}(A \oplus X, y)=\Phi_{e}\left(A \oplus \Psi_{e}(C), y\right)[s]=0,
$$

so $y \notin B$ for the desired contradiction.

\section{A MUlti-Generic SeT}

This section is dedicated to the proof of the following theorem.

Theorem 5.1 (Theorem 1.22). Let $C$ be a c.e. set and let $\left\{A_{i}: i \in \omega\right\}$ be a uniformly $C$-computable list of sets. Then, there exists a set $G \leqslant_{T} C$ such that $G$ is 1-generic relative to $A_{i}$, for every $i$ with $A_{i}<_{T} C$. 
We have to satisfy the following genericity requirements.

$\mathbf{R}_{e, i}$ : Either $A_{i}$ computes $C$, or there is some $\sigma \subset G$ such that either $\sigma \in W_{e}^{A_{i}}$ or no extension of $\sigma$ is in $W_{e}^{A_{i}}$.

If such a $\sigma$ exists, then, in the former case, we say that $\mathbf{R}_{e, i}$ has been satisfied by forcing inside $W_{e}^{A_{i}}$, and in the latter case we say we have forced outside $W_{e}^{A_{i}}$.

During the construction we use a computable enumeration of $C$, and use this enumeration of $C$ to obtain uniform computable approximations $\left\langle A_{i, s}: s \in \omega\right\rangle$ of the sets $A_{i}$. The requirements are associated with levels of the tree of strategies as usual. The requirements will work roughly as follows. Suppose $\alpha$ is in the tree of strategies and $\mathbf{R}_{e, i}=\mathbf{R}_{\alpha}$. Requirement $\mathbf{R}_{\alpha}$ monitors $W_{e}^{A_{i}}$, looking for a possible $\sigma \in W_{e}^{A_{i}}$ so that we can make $\sigma$ an initial segment of $G$ and satisfy the requirement by forcing inside $W_{e}^{A_{i}}$. But not every $\sigma \in W_{e}^{A_{i}}$ will be eligible to be an initial segment of $G$. First, we have to respect the work done by higher priority requirements, and hence $\sigma$ will have to extend a string that we will call $\mathbf{r}(\alpha)$ given to $\mathbf{R}_{\alpha}$ as its input by the requirement of immediately higher priority. Second, since we want to get $G \leqslant_{T} C$, we will only consider strings $\sigma$ that are permitted by $C$ in a sense we will specify later. Third, even if we see that $\sigma \in W_{e}^{A_{i}}$ at a stage $s$, our approximation $A_{i}$ might change later, and $\sigma$ might be removed from this set. So, the plan for $\mathbf{R}_{\alpha}$ is to collect, into a set $P(\alpha)$, pairs $(\tau, \sigma)$, where $\sigma \in W_{e}^{\tau}, \tau$ is a potential initial segment of $A_{i}$, and $\sigma$ is eligible according to certain conditions we will specify later. Then, if we see that one of these strings $\tau$ is actually an initial segment of $A_{i}$, we will try to make $\sigma$ an initial segment of $G$.

As we mentioned above, requirement $\mathbf{R}_{\alpha}$ will be given an input string $\mathbf{r}(\alpha)$ that $\mathbf{R}_{\alpha}$ must keep as an initial segment of $G$. The possible outcomes of $\mathbf{R}_{\alpha}$ are $\infty$ or a pair $(\tau, \sigma) \in\left(2^{<\omega}\right)^{2}$. The outcome $\infty$ means that $\mathbf{R}_{\alpha}$ will be satisfied by forcing outside of $W_{e}^{A_{i}}$. In this case, the next requirement $\mathbf{R}_{\alpha-\infty}$ will receive input $\mathbf{r}(\alpha)$ too. An outcome $(\tau, \sigma) \in\left(2^{<\omega}\right)^{2}$ codes that $\sigma \in W_{e}^{\tau}$, that $\tau \subset A_{i}$, and that $\sigma$ is an initial segment of $G$. Therefore, it is coding that $\mathbf{R}_{\alpha}$ is being satisfied by forcing inside $W_{e}^{A_{i}}$. In this case, the next requirement $\mathbf{R}_{\alpha-(\tau, \sigma)}$ will receive input $\sigma$.

Let us now describe the construction in more detail.

The tree of strategies consists of those finite strings $\alpha$ such that:

- For all $i<|\alpha|$, either $\alpha(i)=\infty$ or $\alpha(i) \in\left(2^{<\omega}\right)^{2}$.

- If $i<j<|\alpha|, \alpha(i)=\left(\tau_{1}, \sigma_{1}\right)$ and $\alpha(j)=\left(\tau_{2}, \sigma_{2}\right)$, then $\sigma_{1} \subseteq \sigma_{2}$.

- If $i<|\alpha|, \alpha(i)=(\tau, \sigma)$ and $\alpha \uparrow i$ works for requirement $R_{e, j}$, then $\sigma \in W_{e}^{\tau}$.

The restraint $\mathbf{r}(\alpha)$ imposed on a node $\alpha$ is the union of all strings $\sigma$ where for some $\tau$ and some $i<|\alpha|, \alpha(i)=(\tau, \sigma)$.

Each node $\alpha$ enumerates auxiliary sets $Q(\alpha, t)$ and $P(\alpha)$. The set $Q(\alpha, t)$ consists of triples $(\tau, \sigma, m)$, indicating that at stage $t, \alpha$ has observed that $(\tau, \sigma)$ would be a desirable outcome, but it still needs a change in $C \uparrow m$ to allow $\alpha$ to pick this pair as an outcome. The set $P(\alpha)$ consists of those desirable pairs $(\tau, \sigma)$ which have been permitted by $C$ to become outcomes of $\alpha$. The set $P(\alpha)$ is computably enumerable, uniformly in $\alpha$. For $s<\omega$, we let $P_{s}(\alpha)$ be the collection of pairs enumerated into $P(\alpha)$ by the beginning of stage $s$.

5.1. Construction. At each stage $s$ we will define an approximation $\alpha_{s}$ to the true path, where $\alpha_{s}$ is a string in the tree of strategies of length $s$. We will use $\mathbf{r}\left(\alpha_{s}\right)$ as our stage-s-approximation to $G$. 
At stage 0 , only the root \langle\rangle is accessible, so $\alpha_{0}=\langle\rangle$. We do not do anything else at stage 0 .

At stage $s>0$, we first inductively decide which nodes $\alpha$ are accessible at stage $s$ (we also say that $s$ is an $\alpha$-stage). Only after we have defined $\alpha_{s}$, the node of length $s$ which is accessible at stage $s$, do we define $Q(\alpha, s)$ and enumerate new elements into $P(\alpha)$, where $\alpha^{\curvearrowleft} \infty$ is accessible at stage $s$.

As usual, the root \langle\rangle is accessible at stage $s$.

Suppose that we have already decided that a node $\alpha$ is accessible at stage $s$. If $|\alpha|=s$, then we halt the stage, and so let $\alpha_{s}=\alpha$.

Suppose that $|\alpha|<s$. Let $|\alpha|=(e, i)$, so $\alpha$ works to meet $\mathbf{R}_{e, i}$.

If there is some pair $(\tau, \sigma)$ in $P_{s}(\alpha)$ such that $\tau \subset A_{i, s}$, then we let $\alpha \frown(\tau, \sigma)$ be the next accessible node; we take no further action for $\alpha$.

Otherwise, we let $\alpha \frown \infty$ be the next accessible node.

This defines $\alpha_{s}$. Now let $\alpha$ be any node such that $\alpha \frown \infty$ is accessible at stage $s$.

Suppose that $\tau \subseteq A_{i, s},|\tau|<s, \sigma \in W_{e}^{\tau}$, and that $\sigma \supseteq \mathbf{r}(\alpha)$. If $\sigma \subseteq \mathbf{r}\left(\alpha_{s}\right)$, then we enumerate $(\tau, \sigma)$ into $P(\alpha)$. Otherwise, we let $m$ be the greatest integer such that $\sigma \uparrow m=\mathbf{r}\left(\alpha_{s}\right) \uparrow m$. We then enumerate $(\tau, \sigma, m)$ into $Q(\alpha, s)$.

Suppose that $(\tau, \sigma, m) \in Q(\alpha, t)$ for some $t<s$, and that $C_{t} \uparrow m \neq C_{s} \uparrow m$. We then enumerate $(\tau, \sigma)$ into $P(\alpha)$.

\subsection{Verifications.}

Lemma 5.2. A true path exists: if $\alpha$ is accessible infinitely often, then either $\alpha^{\frown}(\infty)$ is accessible infinitely often (and for no $(\tau, \sigma)$ is $\alpha^{\frown}(\tau, \sigma)$ accessible infinitely often), or there are strings $\tau, \sigma$ such that from some stage, whenever $\alpha$ is accessible, so is $\alpha^{\frown}(\tau, \sigma)$.

Proof. If for some $(\tau, \sigma) \in P(\alpha)$ we have $\tau \subset A_{i}$, then once the approximation to $A_{i}$ settles on $\tau$, at every $\alpha$-stage we will have that $\alpha^{\frown}(\tau, \sigma)$ is accessible. Otherwise, for every $(\tau, \sigma) \in P(\alpha)$, there will be a point after which $\alpha^{ح}(\tau, \sigma)$ is never accessible. Therefore, for every given $s$, there is a later stage $t$ that is not $\alpha^{\frown}(\tau, \sigma)$ accessible for any $(\tau, \sigma) \in P_{s}(\alpha)$. So, there has to be some $\alpha \frown \infty$-stage after $s$, for every given $s$. Hence $\alpha^{\frown}(\infty)$ is accessible infinitely often and for no $(\tau, \sigma)$ is $\alpha^{\frown}(\tau, \sigma)$ accessible infinitely often.

We define

$$
G=\bigcup\{\mathbf{r}(\alpha): \alpha \in \text { true path }\} .
$$

(To show that $G$ is actually an infinite string one can use the fact that for every $n$ there exists an $e$ such that for every $X, W_{e}^{X}=2^{>n}$, and we will always be able to force inside this set.)

Lemma 5.3. Every requirement $\mathbf{R}_{e, i}$ is met.

Proof. Let $e, i \in \omega$, and suppose that no initial segment of $G$ forces either into or out of $W_{e}^{A_{i}}$. We show that $A_{i} \geqslant_{T} C$.

Let $\alpha$ on the true path work for $\mathbf{R}_{e, i}$. So $\alpha^{\frown} \infty$ is on the true path as well, because otherwise we would have forced inside $W_{e}^{A_{i}}$.

Suppose that at an $\alpha^{\curvearrowright} \infty$-stage $r$, there is some string $\tau$, an initial segment of both $A_{i}$ and of $A_{i, r}$, and some string $\sigma \in W_{e}^{\tau}$ extending both $\mathbf{r}\left(\alpha_{r}\right) \uparrow n$ and $\mathbf{r}(\alpha)$. Then we know that $C \uparrow n=C_{r} \uparrow n$, because otherwise at some later stage we would add $(\tau, \sigma)$ to $P(\alpha)$. 
On the other hand, for each $n$, there are infinitely many such stages, because $W_{e}^{A_{i}}$ is dense around $G$. We can then use $A_{i}$ to find these stages and compute $C$.

The main difficulty in proving that $G$ is computable from $C$ comes from the fact that the true path is not necessarily computable from $C$. This is the most interesting part of the proof. The idea is the following: to compute $G\lceil n$ using $C$, we will wait until a stage $s$ where we are sure that for every stage $t>s, \mathbf{r}\left(\alpha_{s} \uparrow n\right)=\mathbf{r}\left(\alpha_{t} \uparrow n\right)$, and hence $\mathbf{r}\left(\alpha_{s}\lceil n)\right.$ is an initial segment of $G$.

Definition 5.4. We say that a stage $s$ is $n$-correct if, for every $\beta \subset \alpha_{s}\lceil n$ the following conditions hold, where $\gamma=\mathbf{r}\left(\alpha_{s}\lceil n)\right.$ and $|\beta|=\langle e, i\rangle$ :

(1) $C \uparrow|\gamma|=C_{s} \uparrow|\gamma|$.

(2) If $\beta^{\frown}(\tau, \sigma) \subseteq \alpha_{s}$ for some $\tau$ and $\sigma$, then for all $t \geqslant s, \tau \subset A_{i, t}$.

(3) If $\beta^{\frown} \infty \subseteq \alpha_{s}$, then for every $(\tau, \sigma) \in P_{s}(\beta)$, either $\sigma \supseteq \gamma$, or for all $t \geqslant s$, $\tau \not \subset A_{i, t}$.

Further, for all $u<s$, for all $(\tau, \sigma, m) \in Q(\beta, u)$, if $C \uparrow m \neq C_{u} \uparrow m$, then either $(\tau, \sigma) \in P_{s}(\beta)$ or $\sigma \supseteq \gamma$.

As the approximations $\left\langle A_{i, s}\right\rangle$ were obtained from the enumeration $\left\langle C_{s}\right\rangle$, the fact that $C$ is c.e. means that $C$ can compute the modulus function for each approximation $\left\langle A_{i, s}\right\rangle$, uniformly in $i$. This implies that $C$ can decide whether a stage $s$ is $n$-correct or not.

We now prove that such stages exist, and that if $s$ is $n$-correct, then $\mathbf{r}\left(\alpha_{s}\lceil n)\right.$ is an initial segment of $G$.

Lemma 5.5. Suppose that $s$ is $n$-correct. Then for every $t \geqslant s, \mathbf{r}\left(\alpha_{t}\right) \supseteq \mathbf{r}\left(\alpha_{s}\lceil n)\right.$.

Proof. Let $\gamma=\mathbf{r}\left(\alpha_{s}\lceil n)\right.$. By induction on $j \leqslant n$ we prove the following statement:

For every $\alpha_{s} \uparrow(n-j)$-stage $t \geqslant s, \mathbf{r}\left(\alpha_{t}\right) \supseteq \gamma$.

Note that this is enough, since every $t$ is an \langle\rangle -stage, and \langle\rangle$=\alpha_{s} \mid(n-n)$.

For the induction basis $j=0$, it is clear that at every $\alpha_{s}\lceil n$-stage $t$ we have $\alpha_{s}\left\lceil n \subseteq \alpha_{t}\right.$, and hence $\mathbf{r}\left(\alpha_{t}\right) \supseteq \mathbf{r}\left(\alpha_{s}\lceil n)\right.$.

For the induction step, let $\beta=\alpha_{s} \uparrow(n-j)$. Let $\mathbf{R}_{e, i}$ be the requirement for which $\beta$ works.

If $\beta \frown(\tau, \sigma) \subseteq \alpha_{s}$ for some $\tau$ and $\sigma$, then every $\beta$-stage $t \geqslant s$ is also a $\beta \frown(\tau, \sigma)$ stage, and by induction, for every such stage $t$ we have $\gamma \subseteq \mathbf{r}\left(\alpha_{t}\right)$.

So suppose that $\beta^{+}=\beta \frown \infty$; we need to show that if $t \geqslant s$ is a $\beta$-stage but is not a $\beta \frown \infty$-stage, then $\gamma \subseteq \mathbf{r}\left(\alpha_{t}\right)$. Let $t$ be such a stage. Then for some $(\tau, \sigma) \in P_{t}(\beta)$ we have $\beta \frown(\tau, \sigma) \subset \alpha_{t}$, which implies that $\sigma \subseteq \mathbf{r}\left(\alpha_{t}\right)$; so it suffices to show that $\sigma \supseteq \gamma$.

If $(\tau, \sigma) \in P_{s}(\beta)$, then $\sigma \supseteq \gamma$ follows from $\tau \subset A_{i, t}$. Suppose, then, that $(\tau, \sigma)$ is enumerated into $P(\beta)$ at some stage $w \in[s, t)$. There are two possibilities. If $\sigma \supseteq \mathbf{r}\left(\alpha_{w}\right)$, then we note that the node $\beta \frown \infty$ is accessible at stage $w$, and so by induction $\gamma \subseteq \mathbf{r}\left(\alpha_{w}\right)$; so certainly $\sigma \supseteq \gamma$, as required.

Otherwise, there is some $u<w$ and some $m$ such that $(\tau, \sigma, m) \in Q(\beta, u)$, and $C_{w} \uparrow m \neq C_{u} \uparrow m$. If $u<s$, then $(\tau, \sigma) \notin P_{s}(\beta)$ implies that $\sigma \supset \gamma$. If $u \geqslant s$, then we note that $u$ is a $\beta \frown \infty$-stage, and $\sigma \uparrow m=\mathbf{r}\left(\alpha_{u}\right) \uparrow m$. Again by induction, $\mathbf{r}\left(\alpha_{u}\right) \supseteq \gamma$. Since $w>u \geqslant s$ we have $C_{w} \uparrow|\gamma|=C_{u} \uparrow|\gamma|$, so $m>|\gamma|$; we get that $\sigma \supseteq \gamma$. 
Lemma 5.6. For every $n$ there exists a stage $s$ that is $n$-correct.

Proof. Let $\alpha$ be the node of length $n$ that is in the true path. Let $\gamma=\mathbf{r}(\alpha)$.

By induction on $i \leqslant n$ we prove the following statement:

There exists a stage $s_{i}$ such that for every $\alpha \uparrow(n-i)$-stage $t \geqslant s_{i}$, $\mathbf{r}\left(\alpha_{t}\right) \supseteq \gamma$.

For $i=0$, this is clearly true; further, let $s_{0}$ be such that $C \uparrow|\gamma|=C_{s_{0}} \uparrow|\gamma|$. Let $\beta=\alpha\lceil(n-i)$ and suppose that we have shown the claim for $i-1$. Let $\langle e, i\rangle=|\beta|$. If $\beta \frown(\tau, \sigma) \subseteq \alpha$ for some $\tau$ and $\sigma$, then let $s_{i}>s_{i-1}$ be such that for every $t \geqslant s_{i}$, $\tau \subseteq A_{i, t}$. Then, every $\beta$-stage $t \geqslant s_{i}$ is a $\beta \frown(\tau, \sigma)$-stage, and hence by the induction hypothesis, $\mathbf{r}\left(\alpha_{t}\right) \supseteq \gamma$.

Suppose now that $\beta \frown \infty \subseteq \alpha$. We know that for all $(\tau, \sigma) \in P(\beta), \tau \not \subset A_{i}$. Since for every stage $u, Q(\beta, u)$ is finite, there are $\beta$-stages $s_{i}>u_{i}>s_{i-1}$ such that:

- For all $t<s_{i-1}$, for all $(\tau, \sigma, m) \in Q(\beta, t)$, if $(\tau, \sigma) \in P(\beta)$, then $(\tau, \sigma) \in$ $P_{u_{i}}(\beta)$.

- For all $(\tau, \sigma) \in P_{u_{i}}(\beta)$, for all $t \geqslant s_{i}, \tau \not \subset A_{i, t}$.

Suppose that a new pair $(\tau, \sigma)$ is enumerated into $P(\beta)$ at some stage $t \geqslant u_{i}$. If $\sigma \supseteq \mathbf{r}\left(\alpha_{t}\right)$, then as $t$ is a $\beta \frown \infty$-stage and $t \geqslant s_{i-1}$, by induction $\mathbf{r}\left(\alpha_{t}\right) \supseteq \gamma$, so $\sigma \supseteq \gamma$.

Otherwise, $(\tau, \sigma, m) \in Q(j, w)$ for some $\beta^{\frown} \infty$-stage $w<t$ and $C_{t} \uparrow m \neq C_{w} \uparrow m$. Since $t \geqslant u_{i}, w \geqslant s_{i-1}$, so again by induction, $\mathbf{r}\left(\alpha_{w}\right) \supseteq \gamma$. Since $s_{i-1} \geqslant s_{0}$, we know that $C_{t} \uparrow|\gamma|=C_{w} \uparrow|\gamma|$; so $m>|\gamma|$. By definition of $Q(\beta, w), \sigma \uparrow m=\mathbf{r}\left(\alpha_{w}\right) \uparrow m$; so altogether, $\sigma \supseteq \gamma$. We have thus shown that for all $(\tau, \sigma) \in P(\beta) \backslash P_{u_{i}}(\beta)$ we have $\sigma \supseteq \gamma$.

Let $t \geqslant s_{i}$ be a $\beta$-stage. If $t$ is a $\beta \frown \infty$-stage, then by induction, $\gamma \subseteq \mathbf{r}\left(\alpha_{t}\right)$. Otherwise, $\beta \frown(\tau, \sigma)$ is accessible at stage $t$, for some $(\tau, \sigma) \in P(\beta)$ such that $\tau \subseteq A_{i, t}$. Since $t \geqslant s_{i},(\tau, \sigma) \notin P_{u_{i}}(\beta)$. Hence $\gamma \subseteq \sigma$. As $t$ is a $\beta \frown(\tau, \sigma)$-stage, we have $\sigma \subseteq \mathbf{r}\left(\alpha_{t}\right)$. It follows that $\gamma \subseteq \mathbf{r}\left(\alpha_{t}\right)$. This completes the induction for $i$.

An examination of the induction shows that, in fact, for all $i \leqslant n$, for every $\alpha$-stage $t \geqslant s_{i}$, conditions (1)-(3) of Definition 5.4 hold for $\beta=\alpha \uparrow(n-i)$. This shows that every $\alpha$-stage $t \geqslant s_{n}$ is $n$-correct.

To compute $G$, all we need to do is use $C$ to find $n$-correct stages $s$ and then we know that $\mathbf{r}\left(\alpha_{s}\lceil n) \subseteq G\right.$. As the length of $\mathbf{r}\left(\alpha_{s}\lceil n)\right.$ is unbounded with $s$ and $n$, this allows us to compute all of $G$ from $C$.

\section{No LEAST JOIN}

Theorem 6.1 (Theorem 1.23). Let $A$ and $B$ be $\Delta_{2}^{0}$ sets; assume that $B$ is noncomputable and that $B$ does not compute $A$. Suppose that $C$ is a c.e. set which computes both $A$ and $B$. Then there is some $X \leqslant_{T} C$ which does not compute $B$, but such that $B \leqslant_{T} A \oplus X$.

6.1. Discussion. The heart of the argument is its reliance on non-uniformity. That is, we build two sets $X_{0}$ and $X_{1}$, one of which will satisfy the conditions required of the set $X$ of the theorem. To explain why we are driven to this non-uniformity, we first describe a naïve, uniform plan, and show the problem with carrying it out.

So, suppose that we computably approximate a $\Delta_{2}^{0}$ set $X$ such that $B \nless_{T} X$ but $B \leqslant_{T} A \oplus X$. To ensure that $B \nless_{T} X$ we meet, for each $e$, the requirement

$$
R_{e}: \Psi_{e}(X) \neq B
$$


where $\left\langle\Psi_{e}\right\rangle$ is an effective enumeration of all Turing functionals. Here is a plan to meet a single requirement $R_{e}$. Let $\left\langle A_{s}\right\rangle$ and $\left\langle B_{s}\right\rangle$ be computable approximations for $A$ and $B$, respectively.

Suppose that the work we do to meet $R_{e^{\prime}}$ for $e^{\prime}<e$ certifies that $\alpha \subset A, \beta \subset B$, and forces us to make $\xi \subset X$ for some finite binary strings $\alpha, \beta$ and $\xi$; for simplicity, assume that $|\alpha|=|\beta|$. Let $n=|\alpha|$.

Suppose that, for a long time, we see that $A_{s}(n)=i$, and that $B_{s}\lceil n+1$ has also been stable for a while. A certification of $A(n)=i$ will come in the form of a pair of strings $\sigma$ and $\tau$, possible initial segments of $X$, which both extend $\xi$, and which form a $\Psi_{e}$-splitting: $\Psi_{e}(\sigma) \perp \Psi_{e}(\tau)$. If we do not find such a splitting, then we argue that $R_{e}$ is met without work; for if $\Psi_{e}(X)=B$, the assumption that $B$ is not computable will ensure the existence of such a splitting.

Given such a certification, we can make a computable promise, that if $B_{s} \uparrow$ $n+1 \subset B$, then $A(n)=i$. For then we have two possibilities: if $A(n) \neq i$, then a subsequent change in $A_{s}\lceil n+1$ will force a change in $C$, which will allow us to ensure that either $\sigma \subset X$ or $\tau \subset X$, choosing so that we can make $\Psi_{e}(X) \perp B$ and meet the requirement $R_{e}$ (while placing only finitely much restraint on weaker requirements). If $A(n)=i$, then we record this fact, and move to try to compute $A(n+1)$ with oracle $B$ in the same fashion. Since we assume that $A \nless_{T} B$, this process must stop after finitely many iterations.

Now here is the difficulty. To ensure that $B \leqslant_{T} A \oplus X$, we enumerate a Turing functional $\Gamma$ and intend that $\Gamma(A, X)=B$. The challenge is to keep $\Gamma$ a consistent functional. Suppose that we discover a $\Psi_{e}$-splitting $\sigma$ and $\tau$, and receive permission to direct $X$ to extend, say $\sigma$. But in a past life, a different requirement, possibly weaker than $R_{e}$, has asked $X$ to extend $\sigma$, and has enumerated an axiom into $\Gamma$ which is compatible with $\sigma$ and the new version of $A$, but which outputs a prehistoric, and incorrect, version of $B$. This would make $\sigma$ ineligible for usage, and derail our strategy.

Indeed, we are not familiar with any direct way to prevent this occurrence. This is where the non-uniformity gives a way to overcome the problem. We now build $X_{0}$ and $X_{1}$. We ensure that for both $j<2$, we have $X_{j} \leqslant_{T} C$ and $B \leqslant_{T} A \oplus X_{j}$, and we make sure that either $B \Varangle_{T} X_{0}$ or $B \Varangle_{T} X_{1}$. Using a Posner-style trick, it is sufficient to meet the following requirements:

$$
R_{e} \text { : either } \Psi_{e}\left(X_{0}\right) \neq B \text { or } \Psi_{e}\left(X_{1}\right) \neq B \text {. }
$$

Lemma 6.25 below is the driver of the constructions, and explains how the fact that we build two sets rather than just one allows us to overcome the difficulty we outlined. To meet requirement $R_{e}$, we again threaten to compute $A$ from $B$, and this time, $A$-configurations will be certified by two strings, possible initial segments of $X_{0}$ and $X_{1}$ which both diagonalize against $B$ when applying $\Psi_{e}$. What Lemma 6.25 says is that, in this situation, we can pick at least one of these strings as an initial segment of $X_{0}$ or $X_{1}$ without introducing inconsistencies to the functional $\Gamma_{j}$ computing $B$ from $A \oplus X_{j}$. Essentially, the reason we can always find strings $\zeta_{j}$ such that $\Psi_{e}\left(\zeta_{j}\right)$ is incompatible with $B$ and such that for at least one $j<2$ we can pick $\zeta_{j}$ as an initial segment of $X_{j}$ without introducing inconsistencies to $\Gamma_{j}$ is the following. Whenever we decide, at some stage $s$, to act to positively meet a requirement $R_{i}$, for some $j<2$, we let $X_{j}$ extend a string $\zeta_{j}$ as above; at the same time, we reroute the other set $X_{1-j}$ to a completely new configuration that has not previously been seen. In the language of the construction below, we use a 
free extension to define $X_{1-j}$. We will then argue that this action frees $X_{1-j}$ from the burden of old $\Gamma_{1-j}$-computations, allowing Lemma 6.25 to hold beyond stage $s$.

6.2. Preliminaries. As mentioned above, we are given computable approximations $\left\langle A_{s}\right\rangle$ and $\left\langle B_{s}\right\rangle$ for $A$ and $B$ respectively.

6.2.1. The role of $C$. The fact that $C$ is computably enumerable means that we may assume, by choosing appropriate computable approximations for $A$ and $B$, that $C$ can compute a modulus function for $A$ and $B$. Specifically, we let, for $n<\omega, m(n)$ be the least stage $s$ such that for all $t \geqslant s, A_{t}\lceil n+1=A\lceil n+1$ and $B_{t} \uparrow n+1=B\left\lceil n+1\right.$. We pick approximations $\left\langle A_{s}\right\rangle$ and $\left\langle B_{s}\right\rangle$ so that $m \leqslant_{T} C$. From now on, we avoid all reference to $C$ and use the oracle $m$ instead.

The function $m: \omega \rightarrow \omega$ has a left-c.e. approximation. For $s<\omega$, let $m_{s}$ be the stage $s$ approximation for $m: m_{s}(n)$ is the greatest $\bar{s} \leqslant s$ such that for all $t \in[\bar{s}, s]$, we have $A_{t} \uparrow n+1=A_{s}\left\lceil n+1\right.$ and $B_{t} \uparrow n+1=B_{s} \uparrow n+1$. The approximation being "left-c.e." means that if $s<t, \mu \subset m_{s}$ and $\mu \not \subset m_{t}$, then $\mu \not \subset m$; indeed $\mu \not \subset m_{u}$ for any $u \geqslant t$, and for all $s, m_{s}$ lies lexicographically to the left of $m_{s+1}$. We have $m_{s}\left\lceil n \neq m_{s+1}\left\lceil n\right.\right.$ if and only if either $A_{s}\left\lceil n \neq A_{s+1}\left\lceil n\right.\right.$ or $B_{s}\left\lceil n \neq B_{s+1}\lceil n\right.$.

6.2.2. Functionals. Rather than defining $X_{0}$ and $X_{1}$ directly, we enumerate Turing functionals $\Xi_{0}$ and $\Xi_{1}$, and in the end, let, for $j<2, X_{j}=\Xi_{j}(m)$. We mention now that these functionals need not be, strictly speaking, consistent; but we will show that $\Xi_{0}(m), \Xi_{1}(m) \in 2^{\omega}$. See the discussion in Section 6.2.7.

We also enumerate functionals $\Gamma_{0}$ and $\Gamma_{1}$, and will show that for both $j<2$, $B=\Gamma_{j}\left(A, X_{j}\right)$. These functionals will be consistent.

For axioms, we use the following notation: $\mu \mapsto \eta$ means that if $\mu$ is an initial segment of the oracle, then the output extends $\eta$. As $\Gamma_{0}$ and $\Gamma_{1}$ use two oracles, the axioms will be of the form $(\sigma, \eta) \mapsto \beta$, where $\sigma$ is an initial segment of the first oracle, $\eta$ is an initial segment of the second, and $\beta$ is an initial segment of the output.

We thus think, set-theoretically, of $\Gamma_{0}$ and $\Gamma_{1}$ as binary relations, and denote, for $j<2, \operatorname{dom} \Gamma_{j}$, to be the collection of pairs $(\sigma, \tau)$ such that there is some axiom $(\sigma, \eta) \mapsto \beta$ in $\Gamma_{j}$.

At stage $s$, we let $\Xi_{0, s}, \Xi_{1, s}, \Gamma_{0, s}$ and $\Gamma_{1, s}$ be the collection of axioms enumerated into the corresponding functionals by the end of stage $s$.

We note that as the intended oracle of $\Xi_{j}$ is $m$, at every stage $s$, we only enumerate into $\Xi_{j}$ axioms of the form $\mu \mapsto \eta$ where $\mu \subset m_{s}$.

\subsubsection{Procedures. Recall that we try to meet the requirements}

$R_{e}$ : Either $\Psi_{e}\left(X_{0}\right) \neq B$ or $\Psi_{e}\left(X_{1}\right) \neq B$.

To work toward meeting a requirement $R_{e}$, we will, from time to time, appoint a procedure $p$. The procedure may, at some times, call another procedure to work on the next requirement; see Section 6.2.6. The procedure may also be cancelled at a later stage.

The following pieces of information are attached to a procedure.

(1) A number $e_{p}$; this is the index of the requirement toward meeting which procedure $p$ works. 
(2) A (computable) function $\alpha_{p}$. This function records the attempt to compute $A$ from $B$; setting $\sigma=\alpha_{p}(\beta)$ denotes that $p$ has obtained certification that if $\beta \subset B$, then $\sigma \subset A$. We will ensure that for all $\beta \in \operatorname{dom} \alpha_{p},\left|\alpha_{p}(\beta)\right|=|\beta|$.

(3) A finite string $\mu_{p}$. The procedure $p$ guesses that $\mu_{p} \subset m$. Since $\left\langle m_{s}\right\rangle$ is left-c.e., whenever the guess seems incorrect, it is verified to be incorrect, and the procedure is cancelled.

(4) The domain of $\alpha_{p}$ will in fact be a tree of binary strings, with extensions being one-bit extensions. The root of this tree will be a string denoted by $\rho_{p}$; we will ensure that $\left|\rho_{p}\right|=\left|\mu_{p}\right|$. If $\beta \in \operatorname{dom} \alpha_{p}$ is not $\rho_{p}$, then $\beta^{-}=\beta\left\lceil|\beta|-1\right.$, the immediate predecessor of $\beta$ in $2^{<\omega}$, is also in dom $\alpha_{p}$.

(5) Two binary strings $\eta_{p, 0}$ and $\eta_{p, 1}$. The procedure $p$ declares that if it is never cancelled, then $\eta_{p, 0} \subset X_{0}$, and $\eta_{p, 1} \subset X_{1}$.

(6) Suppose that $\beta \in \operatorname{dom} \alpha_{p}$ and $\beta \neq \rho_{p}$. Then the reduction $\sigma=\alpha_{p}(\beta)$ must be certified. This certification comes in the following form: we define a number $k_{p}(\beta)$ and some $j_{p}(\beta)<2$. For every binary string $\delta \supset \beta$ of length $k_{p}(\beta)$, we appoint a binary string $\zeta_{p}(\delta)$. The meaning of this is that if $\beta \subset B$ and $\alpha_{p}(\beta) \not \subset A$, and $\beta$ is minimal with respect to this property, then we want $\zeta_{p}(\delta) \subset X_{j_{p}(\beta)}$, where $\delta$ is the unique extension of $\beta$ of length $k_{p}(\beta)$ which is an initial segment of $B$. This will be useful for diagonalization, because we will require that $\Psi_{e_{p}}\left(\zeta_{p}(\delta)\right) \perp \delta$. The choice of $j_{p}(\beta)$ indicates that the strings $\zeta_{p}(\delta)$ are eligible to be initial segments of $X_{j_{p}(\beta)}$, and that such an appointment would not make the functional $\Gamma_{j_{p}(\beta)}$ inconsistent.

We remark that it seems that for definiteness, we should have used the notation $\zeta_{p, \beta}(\delta)$, rather than merely $\zeta_{p}(\delta)$. The point is that we shall ensure that if $\beta, \beta^{\prime} \in$ dom $\alpha_{p}$ are not $\rho_{p}$, then $k_{p}(\beta) \neq k_{p}\left(\beta^{\prime}\right)$; indeed, $k_{p}(\beta)$ will be chosen large at the stage at which $\beta$ is added to $\operatorname{dom} \alpha_{p}$, and at most one string is added to $\operatorname{dom} \alpha_{p}$ at each stage. Thus $|\delta|=k_{p}(\beta)$ determines $\beta$, and there is no overlap between $\delta \supset \beta$ of length $k_{p}(\beta)$ and $\delta^{\prime} \supset \beta^{\prime}$ of length $k_{p}\left(\beta^{\prime}\right)$, and so no ill-definedness for the expression $\zeta_{p}(\delta)$.

6.2.4. Some notation for strings. For binary strings $\sigma$ and $\tau$, we let $\sigma \subseteq \tau$ denote that $\sigma$ is an initial segment of $\tau$, and $\sigma \subset \tau$ denote that $\sigma$ is a proper initial segment of $\tau$. We let $\sigma \perp \tau$ denote that $\sigma$ and $\tau$ are incomparable.

For a non-empty string $\sigma$, we let $\neg(\sigma)$ be the string of length $|\sigma|$ which agrees with $\sigma$ on all but the last bit.

6.2.5. Free extensions. For any stage $s$, let $\#(s)$ be the largest number used or observed at stage $s$. We may assume that for all $s, \#(s)<\#(s+1)$. A number is called large at stage $s$ if it is greater than \# $(s-1)$.

Whenever we choose a string $\eta_{p, j}$ or $\zeta_{r}(\delta)$ (as a potential initial segment of some $X_{j}$ ), we always choose a string whose last digit is 1 .

Definition 6.2. Let $s<\omega$, and let $\eta$ be any string. A free extension of $\eta$ at stage $s$ is any string of the form $\eta^{\urcorner} 0^{k} 1$, where $k>\#(s-1)$.

Free extensions are useful because no old axioms apply to them:

Lemma 6.3. If $\eta$ is a free extension of $\bar{\eta}$ at stage $s$, and a string $\zeta$ was chosen as a potential initial segment of some $X_{j}$ (that is, as some $\eta_{p, j}$ or $\zeta_{r}(\delta)$ ) at a stage $t<s$, then $\zeta \subseteq \eta$ implies that $\zeta \subseteq \bar{\eta}$. 
Proof. Since $\bar{\eta} \subset \eta$, if $\zeta \subseteq \eta$, then $\zeta \not \perp \bar{\eta}$. Suppose that the lemma fails; then $\bar{\eta} \subset \zeta$. But $|\zeta| \leqslant \#(s-1)$, and the last bit of $\zeta$ is 1 , so if $k>\#(s-1)$ we must have $\zeta \perp \bar{\eta}^{\wedge} 0^{k}$. Then $\zeta \perp \eta$, contradicting our assumption.

Hence, if $\eta$ is a free extension of $\bar{\eta}$ at stage $s$, then for any $\sigma$, we have $\Gamma_{j, s-1}(\sigma, \eta)=$ $\Gamma_{j, s-1}(\sigma, \bar{\eta})$.

6.2.6. The combined tree of procedures. At the end of every stage, there will be finitely many procedures running. These are linearly ordered by a priority ordering: apart from $p_{0}$, the first procedure (which is never cancelled), every procedure is called by the previous procedure on the list. We write $p<q$ to denote that $q$ was called by $p$ or by some descendant of $p$, and thus has weaker priority than $p$. We maintain this nested structure of procedures by following two rules:

- If a procedure $p$ is cancelled at a stage $s$, then every procedure weaker than $p$ is also cancelled at stage $s$.

- Only the weakest procedure running is allowed to call a new procedure.

Say that a procedure $p$ called a procedure $q$. This calling will be done for the benefit of one particular string $\beta \in \operatorname{dom} \alpha_{p}$; the idea is that $q$ guesses that $\beta$ witnesses the success of the procedure $p$ to meet its requirement $R_{e_{p}}$. We will call $p$ the mother of $q$ and $\beta$ the father of $q$, and call $q$ the child of $p$ (and $\beta$ ). To sum up this information, we write parents $(q)=(p, \beta)$.

The following will be useful notation. We let $\mathbb{P}_{s}$ be the collection of pairs $(p, \beta)$ such that $p$ is a procedure which is running at the end of stage $s$, and such that $\beta \in \operatorname{dom} \alpha_{p}$ at the end of stage $s 1$

On $\mathbb{P}_{s}$ we put a partial ordering, which we also denote by $<$. This is the transitive closure of the following two cases:

(1) If $\beta \subset \gamma$ are elements of dom $\alpha_{p}$, then $(p, \beta)<(p, \gamma)$.

(2) If $q$ is not the strongest procedure, then parents $(q)<\left(q, \rho_{q}\right)$.

The fact that the priority ordering linearly orders $\operatorname{dom} \mathbb{P}_{s}$, the collection of procedures which are running at the end of stage $s$, and that ancestry implies stronger priority, implies that $<$ is indeed a partial ordering on $\mathbb{P}_{s}$. The fact that $p<q$ iff $p$ can be obtained from $q$ by a sequence of ancestries implies that the image of $<$ under the projection map $(p, \beta) \mapsto p$ is exactly the priority ordering on $\operatorname{dom} \mathbb{P}_{s}$.

In fact, the map $p \mapsto\left(p, \rho_{p}\right)$ is an order-preserving injection of dom $\mathbb{P}_{s}$ into $\mathbb{P}_{s}$. So we sometimes identify $p$ with $\left(p, \rho_{p}\right)$.

We let $\mathbb{Q}_{s}$ be the collection of pairs $(p, \beta)$ in $\mathbb{P}_{s}$ such that $\beta \subset B_{s}$ and $\alpha_{p}(\beta) \subset A_{s}$. Recall that $\beta$ guesses that $\beta \subset B$, and $\beta$ and $p$ guess together that $\alpha_{p}(\beta) \subset A$. Thus $\mathbb{Q}_{s}$ is the collection of pairs $(p, \beta) \in \mathbb{P}_{s}$ which seem, at stage $s$, to be guessing correctly.

6.2.7. The pseudo-consistency of $\Xi_{j}$. Strictly speaking, the functionals $\Xi_{0}$ and $\Xi_{1}$ need not be consistent: it is possible, for example, that at some stage $t$, we enumerate an axiom $m_{t} \uparrow k \mapsto \zeta$ into $\Xi_{j}(j<2)$, and at a later stage $s$ such that $m_{s}\left\lceil k \neq m_{t}\left\lceil k\right.\right.$, we enumerate an axiom $m_{s}\left\lceil n \mapsto \zeta^{\prime}\right.$ into the same $\Xi_{j}$, with $n<k$, $m_{s}\left\lceil n \subset m_{t} \uparrow k\right.$, and $\zeta^{\prime} \perp \zeta$.

\footnotetext{
${ }^{1}$ For any $t$ we let $\mathbb{P}_{<t}=\bigcup_{s<t} \mathbb{P}_{s}, \mathbb{P}_{<\omega}=\bigcup_{s} \mathbb{P}_{s}$, etc.
} 
The point is that the approximation $\left\langle m_{s}\right\rangle$ of $m$ is left-c.e.; since $m_{s}$ moved to the right of $m_{t}\left\lceil k\right.$, we know that $m_{t}\lceil k$ is not an initial segment of $m$, and so at stage $s$ we can consider the axiom $m_{t} \uparrow k \mapsto \zeta$ as if it's been discarded from $\Xi_{j}$. Formally, what we will actually want is that $\Xi_{j}(m) \in 2^{\omega}$.

Lemma 6.4. Let $j<2$. Suppose that for all $s, \Xi_{j, s}\left(m_{s}\right) \in 2^{<\omega}$. Then $\Xi_{j}(m) \in$ $2^{\leqslant \omega}$.

Proof. Standard, for those familiar with working with left-c.e. oracles. Say for $i<2$, both axioms $m\left\lceil n_{i}, \mapsto \zeta_{i}\right.$ are in $\Xi_{j}$. For sufficiently late $s$, for both $i<2$, $m\left\lceil n_{i} \subset m_{s}\right.$, and the axiom $m\left\lceil n_{i} \mapsto \zeta_{i}\right.$ is in $\Xi_{j, s}$. Thus $\zeta_{0}, \zeta_{1} \subseteq \Xi_{j, s}\left(m_{s}\right)$, and hence are comparable.

We will need to prove that $\Xi_{j, s}\left(m_{s}\right) \in 2^{<\omega}$ during the verification (Section 6.4.5). However, we will need this consistency in order for the instructions of the construction to make sense. Circularity will be avoided by a step-by-step induction.

\section{To perform stage $s$ of the construction, we assume that $\Xi_{j, s-1}\left(m_{s-1}\right) \in 2^{<\omega}$.}

Lemma 6.5. If $\Xi_{j, s-1}\left(m_{s-1}\right) \in 2^{<\omega}$, then $\Xi_{j, s-1}\left(m_{s}\right) \in 2^{<\omega}$.

Proof. Since the approximation $\left\langle m_{s}\right\rangle$ is left-c.e., and since at all stages $t<s$, we only enumerate axioms of the form $m_{t}\left\lceil n \mapsto \zeta\right.$ into $\Xi_{j}$, we have

$$
\Xi_{j, s-1}\left(m_{s}\right) \subseteq \Xi_{j, s-1}\left(m_{s-1}\right) .
$$

For brevity, we let, for $n<\omega, \xi_{j, t, n}=\Xi_{j, t}\left(m_{t}\lceil n)\right.$ and $\xi_{j, t, n}^{*}=\Xi_{j, t-1}\left(m_{t}\lceil n)\right.$. The proof of Lemma 6.5 shows:

Lemma 6.6. If $\Xi_{j, s-1}\left(m_{s-1}\right) \in 2^{<\omega}$, then for all $n, \xi_{j, s-1, n} \in 2^{<\omega}$ and $\xi_{j, s, n}^{*} \in$ $2^{<\omega}$; indeed $\xi_{j, s, n}^{*} \subseteq \xi_{j, s-1, n}$.

\subsection{Construction.}

At stage 0 , we call the first procedure $p_{0}$, set $e_{p_{0}}=0, \mu_{p_{0}}=\langle\rangle$, let $\rho_{p_{0}}=\langle\rangle$, and define $\alpha_{p_{0}}(\langle\rangle)=\langle\rangle$, and $\eta_{p_{0}, 0}=\eta_{p_{0}, 1}=\langle\rangle$.

Let $s>0$. Stage $s$ consists of three steps.

1. Cancelling procedures. We cancel every procedure $p$ such that $\mu_{p} \not \subset m_{s}$.

2. Extending trees. Let $p \in \operatorname{dom} \mathbb{P}_{s-1}$ be a procedure which is still running, i.e., was not cancelled at step 1 , and suppose that it is not the weakest such procedure; let $q$ be the child of $p$. Let $\beta$ be the longest initial segment of $B_{s}$ in dom $\alpha_{p}$ such that $\alpha_{p}(\beta) \subset A_{s}$ 2 Let $\beta^{+}=B_{s}\left\lceil|\beta|+1=\beta^{\frown} B_{s}(|\beta|)\right.$, and let $\alpha^{+}=A_{s} \uparrow|\beta|+1=$ $\alpha_{p}(\beta)^{\frown} A_{s}(|\beta|)$. Let $j<2$ be such that there are no $\sigma \supseteq \neg\left(\alpha^{+}\right)$and $\zeta \subseteq \eta_{q, j}$ such that $(\sigma, \zeta) \in \operatorname{dom} \Gamma_{j, s-1} 3^{3}$

Let $k$ be large. The procedure $p$ requires attention if $\left(p, \beta^{+}\right) \notin \mathbb{P}_{s-1}$, and for every $\gamma \supset \beta^{+}$of length $k$ we can find a long string $\zeta_{\gamma} \supset \eta_{q, j}$ such that $\Psi_{e_{p}}\left(\zeta_{\gamma}\right) \perp \gamma$.

\footnotetext{
${ }^{2} \beta$ is the longest string such that $(p, \beta)$ is in both $\mathbb{P}_{s-1}$ and $\mathbb{Q}_{s}$, unless $p$ is cancelled at stage $s$. Lemma 6.15 ensures the existence of $\beta$.

${ }^{3}$ Corollary 6.26 ensures the existence of such a $j$. We do not need to assume the lemma holds for the construction to make sense; if there were no such $j, p$ simply would not require attention.
} 
If there is a procedure which requires attention, let $p$ be the strongest one. We then do the following:

(1) Set $k_{p}\left(\beta^{+}\right)=k$ and $j_{p}\left(\beta^{+}\right)=j$; for every $\gamma \supset \beta^{+}$of length $k$, let $\zeta_{p}(\gamma)=\zeta_{\gamma}$.

(2) Define $\alpha_{p}\left(\beta^{+}\right)=\alpha^{+}$.

(3) Cancel all procedures weaker than $p$; end stage $s$.

3. Calling a new procedure. If no procedure requires attention, let $p$ be the weakest procedure running. Define $\beta$ and $\beta^{+}$as in step 2 .

We define an integer $k$ as follows:

- If $\left(p, \beta^{+}\right) \notin \mathbb{P}_{s-1}$, let $t$ be the least stage such that $(p, \beta) \in \mathbb{P}_{t}$, and for all $u \in[t, s],(p, \beta) \in \mathbb{Q}_{u} ;$ let $k=\#(t)+14$

- Otherwise, we let $k=k_{p}\left(\beta^{+}\right)$.

Let $\gamma=B_{s} \uparrow k$. We call a new procedure $q$, weaker than $p$. We set it up as follows:

(1) $e_{q}=e_{p}+1$.

(2) $\mu_{q}=m_{s} \uparrow k$.

(3) $\operatorname{parents}(q)=(p, \beta)$.

(4) $\rho_{q}=\gamma$ and $\alpha_{q}(\gamma)=A_{s}\lceil k$.

(5) If $\left(p, \beta^{+}\right) \notin \mathbb{P}_{s-1}$, then for both $j<2$, we let $\eta_{q, j}$ be some free extension of $\xi_{j, s, k}^{*}$.

(6) If $\left(p, \beta^{+}\right) \in \mathbb{P}_{s-1}$, then for $j=j_{p}\left(\beta^{+}\right)$, we let $\eta_{q, j}$ be some free extension of $\zeta_{p}(\gamma)$; for $j \neq j_{p}\left(\beta^{+}\right)$, we let $\eta_{q, j}$ be some free extension of $\xi_{j, s,|\beta|}^{*}$.

We also enumerate new axioms: for both $j<2$, we enumerate the axiom $\mu_{q} \mapsto \eta_{q, j}$ into $\Xi_{j}$, and the axiom $\left(\alpha_{q}\left(\rho_{q}\right), \eta_{q, j}\right) \mapsto \rho_{q}$ into $\Gamma_{j}$.

6.4. Verification. The verification has four main parts. First, we prove that for both $j<2, \Xi_{j}(m)$ is consistent, thus showing that the construction can be carried out at every stage. Then, we show that both functionals $\Gamma_{0}$ and $\Gamma_{1}$ are consistent. Third, we prove the main lemma (Lemma 6.25), which states exactly that the fact that we build two sets $X_{0}$ and $X_{1}$ rather than just one allows us to find suitable initial segments for either $X_{0}$ or $X_{1}$, free from historical computations, that will meet the requirements. At the end, we use the main lemma to show that every requirement is met.

Our first goal is to show that the construction can indeed be carried out: that for every stage $s$, for both $j<2, \Xi_{j, s}\left(m_{s}\right) \in 2^{<\omega}$. We will need a little more:

Proposition 6.7. For every stage $s$, for both $j<2$,

(1) $\Xi_{j, s}\left(m_{s}\right) \in 2^{<\omega}$.

(2) For all $p \in \operatorname{dom} \mathbb{P}_{s}, \xi_{j, s,\left|\rho_{p}\right|}=\eta_{p, j}$.

Proposition 6.7 will be proved by induction on $s$. It holds for $s=0$. We fix a stage $s>0$, and assume that the proposition holds at every stage $t<s$; and so the construction can be performed, up to and including stage $s$.

We prove that Proposition 6.7 holds at stage $s$ by proving a series of lemmas about the construction at stage $s$. These are all proved simultaneously, as part of the induction proving Proposition 6.7 That is, for every lemma we prove on the way to the proof of Proposition 6.7 (in Section 6.4.5), we assume right now that the lemma holds at every stage $t<s$, and prove it for stage $s$.

\footnotetext{
${ }^{4}$ If $t=s$, then instead of \# $(s)$, we take the greatest number seen so far in the construction.
} 
The proof of Proposition 6.7 at stage $s$ relies on two lemmas.

Lemma 6.8. Let $p, q \in \operatorname{dom} \mathbb{P}_{s}$, and suppose that $p<q$. Then for both $j<2$, $\eta_{p, j} \subset \eta_{q, j}$.

Lemma 6.9. Suppose that $q \in \operatorname{dom} \mathbb{P}_{<s} \backslash \operatorname{dom} \mathbb{P}_{s}$, but that $\mu_{q} \subset m_{s}$. Suppose that the procedure $p$ is called at stage $s$. Then for both $j<2, \eta_{q, j} \subset \eta_{p, j}$.

The point is the following: to show that $\Xi_{j, s}\left(m_{s}\right)$ is consistent, we need to show that if $p$ is called at stage $s$, then the axiom $\mu_{p} \mapsto \eta_{p, j}$, which is enumerated into $\Xi_{j}$ at stage $s$, is consistent with an older axiom $\mu_{q} \mapsto \eta_{q, j}$ in $\Xi_{j, s-1}$ which still applies to $m_{s}$, i.e. such that $\mu_{q} \subset m_{s}$. We need to show that $\eta_{p, j}$ and $\eta_{q, j}$ are comparable. How to do so depends on whether $q$ is still running at the end of stage $s$. If it is, Lemma 6.8 does the trick; if not, we need Lemma 6.9.

We prove Lemma 6.8 in Section 6.4.3 and Lemma 6.9 in Section 6.4.4.

6.4.1. Basic facts. We start by proving several facts about the construction.

Lemma 6.10. $p_{0}$ is not cancelled at stage $s$.

Proof. Because \langle\rangle$\subset m_{s}$.

Lemma 6.11. For all $(p, \beta) \in \mathbb{P}_{s}$, if $\beta \neq \rho_{p}$, then $k_{p}(\beta)>|\beta|$.

Proof. $k_{p}(\beta)$ is chosen to be large, at a stage $t \leqslant s$ such that $(p, \beta) \in \mathbb{P}_{t-1}$. Hence $k_{p}(\beta)>\#(t-1) \geqslant|\beta|$.

Lemma 6.12. Suppose that $q \neq p_{0}$ and $q \in \operatorname{dom} \mathbb{P}_{s}$. Then the length of $\rho_{q}$ is greater than the length of the father of $q$.

Proof. Let $(p, \beta)=$ parents $(q)$; let $t \leqslant s$ be the stage at which $q$ is called; let $k=$ $\left|\rho_{q}\right|$. If $\left(p, \beta^{+}\right) \in \mathbb{P}_{t-1}$, then $k=k_{p}\left(\beta^{+}\right)>\left|\beta^{+}\right|>|\beta|$ by Lemma 6.11. Otherwise, $k>\#(u)$, where $u \leqslant t$ is a stage such that $(p, \beta) \in \mathbb{P}_{u}$; so $\#(u) \geqslant|\beta|$.

Corollary 6.13. If $(p, \beta),(q, \gamma) \in \mathbb{P}_{s}$ and $(p, \beta)<(q, \gamma)$, then $\beta \subset \gamma$.

Proof. We show that if $\beta$ is the father of $q$, then $\beta \subset \rho_{q}$; the rest is immediate. So let $q \in \operatorname{dom} \mathbb{P}_{s}, q \neq p_{0}$, and let $(p, \beta)=$ parents $(q)$. Let $t \leqslant s$ be the stage at which $q$ is called. At stage $t$, we define $\rho_{q} \subset B_{t}$. We also have $\beta \subset B_{t}$. Since $|\beta|<\left|\rho_{q}\right|$ (Lemma 6.12), we get $\beta \subset \rho_{q}$.

Lemma 6.14. If $p, q \in \operatorname{dom} \mathbb{P}_{s}$ and $p<q$, then $\mu_{p} \subset \mu_{q}$.

Proof. Let $t \leqslant s$ be the stage at which $q$ is called. Since $p \in \operatorname{dom} \mathbb{P}_{t}$, we have $\mu_{p} \subset m_{t}$; we also have $\mu_{q} \subset m_{t}$. We have $\left|\mu_{p}\right|=\left|\rho_{p}\right|$ and $\left|\mu_{q}\right|=\left|\rho_{q}\right|$; by Corollary 6.13. $\rho_{p} \subset \rho_{q}$, so $\left|\rho_{p}\right|<\left|\rho_{q}\right|$.

6.4.2. Comparability of $\alpha$ 's, and $\mathbb{Q}_{s}$.

Lemma 6.15. If $p \in \operatorname{dom} \mathbb{P}_{s}$, then $\left(p, \rho_{p}\right) \in \mathbb{Q}_{s}$.

Proof. For $p=p_{0}$, we always have \langle\rangle$\subset B_{s}$ and \langle\rangle$\subset A_{s}$.

Suppose that $p \neq p_{0}$. Let $t \leqslant s$ be the stage at which $p$ was called; let $k=\left|\rho_{p}\right|$. At stage $t$, we set $\rho_{p}=B_{s} \uparrow k$ and $\alpha_{p}\left(\rho_{p}\right)=A_{s} \uparrow k$; we also set $\mu_{p}=m_{s} \uparrow k$. So $\left(p, \rho_{p}\right) \in \mathbb{Q}_{t}$. If $t=s$ we are done. Otherwise, by induction, $\left(p, \rho_{p}\right) \in \mathbb{Q}_{s-1}$. If $\left(p, \rho_{p}\right) \notin \mathbb{Q}_{s}$, then either $\rho_{p} \not \subset B_{s}$ or $\alpha_{p}\left(\rho_{p}\right) \not \subset A_{s}$. Then either $B_{s-1} \uparrow k \neq B_{s} \uparrow k$ or $A_{s-1}\left\lceil k \neq A_{s}\left\lceil k\right.\right.$. In either case, we get $m_{s-1}\left\lceil k \neq m_{s} \uparrow k\right.$. Hence $\mu_{p} \not \subset m_{s}$; so $p$ is cancelled at step 1 of stage $s$, contradicting $p \in \operatorname{dom} \mathbb{P}_{s}$. 
Lemma 6.16. If $(p, \beta),(q, \gamma) \in \mathbb{P}_{s}$ and $(p, \beta)<(q, \gamma)$, then $\alpha_{p}(\beta) \subset \alpha_{q}(\gamma)$.

Proof. We prove two cases:

(1) If parents $(q)=(p, \beta)$, then $\alpha_{p}(\beta) \subset \alpha_{q}\left(\rho_{q}\right)$.

(2) If $\beta^{+}$is an immediate successor of $\beta$ in $\operatorname{dom} \alpha_{p}$, then $\alpha_{p}(\beta) \subset \alpha_{p}\left(\beta^{+}\right)$.

For (1), let $t \leqslant s$ be the stage at which $q$ was called. We have $(p, \beta) \in \mathbb{Q}_{t}$, so $\alpha_{p}(\beta) \subset A_{t}$. We also define $\alpha_{q}\left(\rho_{q}\right)=A_{t} \uparrow\left|\rho_{q}\right|$. Since $\left|\alpha_{p}(\beta)\right|=|\beta|$ and $\left|\rho_{q}\right|>|\beta|$ (Lemma 6.12), we get the desired result.

For (2), let $t \leqslant s$ be the stage at which $\beta^{+}$was added to $\operatorname{dom} \alpha_{p}$. Again, we have $\alpha_{p}(\beta) \subset A_{t}$ and define $\alpha_{p}\left(\beta^{+}\right) \subset A_{t}$; we also have $\left|\alpha_{p}(\beta)\right|=|\beta|$, whereas $\left|\alpha_{p}\left(\beta^{+}\right)\right|=\left|\beta^{+}\right|=|\beta|+1$.

Corollary 6.17. If $(q, \gamma) \in \mathbb{Q}_{s},(p, \beta) \in \mathbb{P}_{s}$, and $(p, \beta)<(q, \gamma)$, then $(p, \beta) \in \mathbb{Q}_{s}$.

Proof. By Corollary 6.13, $\beta \subset \gamma$. By Lemma 6.16, $\alpha_{p}(\beta) \subset \alpha_{q}(\gamma)$. Since $\gamma \subset B_{s}$ and $\alpha_{q}(\gamma) \subset A_{s}$, we get $\beta \subset B_{s}$ and $\alpha_{p}(\beta) \subset A_{s}$.

Thus, $\mathbb{Q}_{s}$ is a linearly ordered initial segment of $\mathbb{P}_{s}$.

Lemma 6.18. Suppose that $q \in \operatorname{dom} \mathbb{P}_{s}, q \neq p_{0}$. Let $(p, \beta)=\operatorname{parents}(q)$. Then $\beta$ is the longest string such that $(p, \beta) \in \mathbb{Q}_{s}$.

Proof. We have $(p, \beta)<\left(q, \rho_{q}\right)$ and $\left(q, \rho_{q}\right) \in \mathbb{Q}_{s}$ (Lemma 6.15). By Corollary 6.17. we get that $(p, \beta) \in \mathbb{Q}_{s}$.

Let $t$ be the stage at which $q$ was called. By design, the lemma holds at stage $t$. Suppose that $t<s$; by induction, the lemma holds at stage $s-1$. If the lemma fails at stage $s$, then $\left(p, \beta^{+}\right) \in \mathbb{Q}_{s}$, where $\beta^{+}=B_{s} \uparrow|\beta|+1$.

The fact that $\left(p, \beta^{+}\right) \in \mathbb{P}_{s}$ and that $q \in \operatorname{dom} \mathbb{P}_{s}$ implies that $\left(p, \beta^{+}\right) \in \mathbb{P}_{s-1}$; otherwise, adding $\beta^{+}$to $\operatorname{dom} \alpha_{p}$ at stage $s$ would cancel $q$ at stage $s$. Now, the fact that $\left(p, \beta^{+}\right) \in \mathbb{Q}_{s} \backslash \mathbb{Q}_{s-1}$ implies that $m_{s} \uparrow\left|\beta^{+}\right| \neq m_{s-1} \uparrow\left|\beta^{+}\right|$. Since $|\beta|<\left|\rho_{q}\right|$ (Lemma 6.12) and $\left|\beta^{+}\right|=|\beta|+1$, we get $m_{s} \uparrow\left|\rho_{q}\right| \neq m_{s-1} \uparrow\left|\rho_{q}\right|$, which would imply that $q$ gets cancelled at step 1 of stage $s$. This is a contradiction.

6.4.3. Comparability of $\eta$ 's.

Lemma 6.19. If $p$ is in both $\operatorname{dom} \mathbb{P}_{s-1}$ and $\operatorname{dom} \mathbb{P}_{s}$, then for both $j<2, \xi_{j, s,\left|\rho_{p}\right|}^{*}=$ $\eta_{p, j}$.

Proof. The assumption implies that $m_{s}\left\lceil\left|\rho_{p}\right|=m_{s-1}\left\lceil\left|\rho_{p}\right|=\mu_{p}\right.\right.$. Hence $\xi_{j, s,\left|\rho_{p}\right|}^{*}=$ $\xi_{j, s-1,\left|\rho_{p}\right|}$. So the conclusion follows from our assumption that Proposition 6.7 (2) holds at stage $s-1$.

Proof of Lemma 6.8. As by induction we assume that the lemma holds at every stage $t<s$, it remains to prove that if $q$ is called at stage $s$, and $p$ is the mother of $q$, then for both $j<2$ we have $\eta_{p, j} \subset \eta_{q, j}$.

Let $\beta$ be the father of $q$. There are two cases.

The first case is when $\eta_{q, j}$ is chosen to be a free extension of $\xi_{j, s,|\beta|}^{*}$ or of $\xi_{j, s,\left|\rho_{q}\right|}^{*}$. We note that $(p, \beta) \in \mathbb{P}_{s-1}$, so $p \in \operatorname{dom} \mathbb{P}_{s-1}$, dom $\mathbb{P}_{s}$, so by Lemma 6.19, $\eta_{p, j}=$ $\xi_{j, s,\left|\rho_{p}\right|}^{*}$. Of course $\xi_{j, s,\left|\rho_{p}\right|} \subseteq \xi_{j, s,|\beta|}^{*}, \xi_{j, s,\left|\rho_{q}\right|}^{*}$.

The second case is when $\eta_{q, j}$ is chosen to be a free extension of $\zeta_{p}\left(\rho_{q}\right)$. Let $\beta^{+}=\rho_{q}\left\lceil|\beta|+1=B_{s} \uparrow|\beta|+1\right.$; in this case we have $\left(p, \beta^{+}\right) \in \mathbb{P}_{s-1}$. Let $t<s$ be the stage at which $\beta^{+}$was added to $\operatorname{dom} \alpha_{p}$, and let $\bar{q}$ be $p$ 's child at stage $t$. By induction, we have $\eta_{p, j} \subset \eta_{\bar{q}, j}$. Chosen at stage $t, \zeta_{p}\left(\rho_{q}\right)$ is required to extend $\eta_{\bar{q}, j}$, so $\zeta_{p}\left(\rho_{q}\right)$ extends $\eta_{p, j}$. Again we get the desired result. 
6.4.4. What happens when $q \in \operatorname{dom} \mathbb{P}_{<s} \backslash \operatorname{dom} \mathbb{P}_{s}$ but $\mu_{q} \subset m_{s}$. In this section we prove Lemma 6.9.

Lemma 6.20. Let $q \in \operatorname{dom} \mathbb{P}_{<s} \backslash \operatorname{dom} \mathbb{P}_{s}$ such that $\mu_{q} \subset m_{s}$. Let $t<s$ be a stage such that $q \in \operatorname{dom} \mathbb{P}_{t}$, and let $r$ be the weakest procedure in $\operatorname{dom} \mathbb{P}_{t}$ which is also in $\operatorname{dom} \mathbb{P}_{s}$. Let $\bar{q}$ be $r$ 's child at stage $t$, and let $u$ be the stage at which $\bar{q}$ is cancelled. Let $\beta$ be $\bar{q}$ 's father.

(1) At stage $u$, a one-bit extension $\beta^{+}$of $\beta$ is added to $\operatorname{dom} \alpha_{r}$, and $\left(r, \beta^{+}\right) \in$ $\mathbb{P}_{s}$.

(2) If a procedure $p$ is called at stage $s$, then $\left|\rho_{p}\right|>\left|\rho_{q}\right|$.

(3) For both $j<2, \eta_{q, j}=\xi_{j, s,\left|\rho_{q}\right|}^{*}$.

(4) If $p \in \operatorname{dom} \mathbb{P}_{s}$ and $(r, \gamma)=\operatorname{parents}(p)$, then $(r, \gamma) \notin \mathbb{P}_{u-1}$.

(5) If $(r, \gamma) \in \mathbb{P}_{s} \backslash \mathbb{P}_{u-1}$ and $|\gamma| \geqslant\left|\rho_{q}\right|$, then for all $\delta \supset \gamma$ of length $k_{r}(\gamma)$, we have $\zeta_{r}(\delta) \supset \eta_{q, j_{r}(\gamma)}$.

Note that $r$ exists, since $p_{0} \in \operatorname{dom} \mathbb{P}_{t}$ and in $\operatorname{dom} \mathbb{P}_{s}$. The procedure $\bar{q}$ exists because $q \in \operatorname{dom} \mathbb{P}_{t}$ and $q \notin \operatorname{dom} \mathbb{P}_{s}$, so $r<q$; so $r$ is not the weakest procedure in $\operatorname{dom} \mathbb{P}_{t}$. By the maximality of $r, \bar{q} \notin \operatorname{dom} \mathbb{P}_{s}$, so $u$ exists, and $u \in(t, s]$.

Proof. Since $r<q$, we have $\bar{q} \leqslant q$. Hence (Lemma 6.14), $\mu_{\bar{q}} \subseteq \mu_{q}$. Since $\mu_{\bar{q}} \subset m_{t}$, we must have $\mu_{\bar{q}} \subset m_{u}$. It follows that $\bar{q}$ was cancelled not at step 1 of stage $u$, but because at stage $u$, some string is added to $\operatorname{dom} \alpha_{r}$.

By Lemma 6.18, $\beta$ is the maximal string such that $(r, \beta) \in \mathbb{Q}_{t}$. Because $|\beta|<\left|\rho_{q}\right|$ (Corollary 6.13), as $(r, \beta)<\left(q, \rho_{q}\right)$ ), we get $m_{t} \uparrow|\beta|+1=m_{u} \uparrow|\beta|+1=m_{s} \uparrow$ $|\beta|+1$. It follows that $\beta$ is the longest string such that $(r, \beta)$ is in both $\mathbb{P}_{u-1}$ and $\mathbb{Q}_{u}$. Hence, at stage $u$, the string $\beta^{+}=B_{u} \uparrow|\beta|+1$ is added to $\operatorname{dom} \alpha_{r}$. By the definition of $\alpha_{r}\left(\beta^{+}\right)$, we have $\left(r, \beta^{+}\right) \in \mathbb{Q}_{u}$. Since $m_{u}\left\lceil|\beta|+1=m_{s}\lceil|\beta|+1\right.$, we get $\left(r, \beta^{+}\right) \in \mathbb{Q}_{s}$, establishing (11).

For (2), suppose that a procedure $p$ is called at stage $s$. Let $\bar{p}$ be the child of $r$ at stage $s$; so $\bar{p} \leqslant p$. If $\bar{p}<p$, then, since $\bar{p}$ was called after stage $u$ but before stage $s$, we have, by induction, $\left|\rho_{\bar{p}}\right|>\left|\rho_{q}\right|$. By Corollary 6.13, we have $\rho_{\bar{p}} \subset \rho_{p}$, so we're done in this case.

Suppose then that $r$ is $p$ 's mother; let $\delta$ be $p$ 's father. The string $\delta$ is the longest string such that $(r, \delta) \in \mathbb{Q}_{s}$. Since $\left(r, \beta^{+}\right) \in \mathbb{Q}_{s}$, we have $\beta^{+} \subseteq \delta$.

Let $\delta^{+}=B_{s} \uparrow|\delta|+1$, and let $k=\left|\rho_{p}\right|$. If $\left(r, \delta^{+}\right) \in \mathbb{P}_{s-1}$, then $k=k_{r}\left(\delta^{+}\right)$. Since $\delta^{+}$is added to dom $\alpha_{r}$ after stage $u$, and $k_{r}\left(\delta^{+}\right)$is chosen large, we have $k_{r}\left(\delta^{+}\right)>\#(u)>\#(t) \geqslant\left|\rho_{q}\right|$, as required.

Suppose that $\left(r, \delta^{+}\right) \notin \mathbb{P}_{s-1}$. We have $k=\#(v)+1$ for some stage $v$ such that $(r, \delta) \in \mathbb{P}_{v}$. Again, since $\delta \supseteq \beta^{+}, \delta$ is added to dom $\alpha_{r}$ not before stage $u$; so $v \geqslant u$. Again we get $k>\#(u)>\#(t) \geqslant\left|\rho_{q}\right|$.

It follows that if $p$ is called at stage $s$, then $\rho_{q} \subset \rho_{p}$ (as they are both extended by $\left.B_{s}\right), \alpha_{q}\left(\rho_{q}\right) \subset \alpha_{p}\left(\rho_{p}\right)$ (they are both extended by $A_{s}$ ), and $\mu_{q} \subset \mu_{p}$ (they are both extended by $\left.m_{s}\right)$.

Let $j<2$. We show that $\xi_{j, s,\left|\rho_{q}\right|}^{*}=\xi_{j, t,\left|\rho_{q}\right|}$. If $\mu \subseteq m_{t} \uparrow\left|\rho_{q}\right|$, then $\mu \subset m_{s}$; so if $\mu \mapsto \zeta$ is in $\Xi_{j, t}$, then $\zeta \subseteq \xi_{j, s,\left|\rho_{q}\right|}^{*}$; so $\xi_{j, t,\left|\rho_{q}\right|} \subseteq \xi_{j, s,\left|\rho_{q}\right|}^{*}$.

On the other hand, suppose that $\mu_{p} \mapsto \eta_{p, j}$ is an axiom which is enumerated into $\Xi_{j}$ at a stage $v \in(t, s)$. Applying part (2) at stage $v$, we get $\left|\mu_{p}\right|>\left|\rho_{q}\right|$, so this new axiom does not apply to the computation $\Xi_{j, s-1}\left(m_{s} \uparrow\left|\rho_{q}\right|\right)$. Hence we get the desired equality. 
Now by induction (Proposition 6.7(2) at stage $t<s$ ), $\eta_{q, j}=\xi_{j, t,\left|\rho_{q}\right|}$, which establishes (3).

Part (4) follows from the fact that $\left(r, \beta^{+}\right) \in \mathbb{Q}_{s}$. By Lemma 6.18, $\beta^{+} \subseteq \gamma$. Since $\beta^{+}$was added to $\operatorname{dom} \alpha_{r}$ at stage $u>t, \gamma$ must have been added at a stage no earlier than $u$.

Finally, we turn to part (5). Let $v \in[u, s]$ be the stage at which $\gamma$ is added to $\operatorname{dom} \alpha_{r}$. Let $o$ be $r$ 's child at stage $v-1$. Let $j=j_{r}(\gamma)$. Let $\delta \supset \gamma$ have length $k_{r}(\gamma)$. At stage $v$, we pick $\zeta_{r}(\delta)$ to extend $\eta_{o, j}$.

- By part (3), applied at stage $v \leqslant s, \eta_{q, j}=\xi_{j, v,\left|\rho_{q}\right|}^{*}$.

- We have $\xi_{j, v,\left|\rho_{q}\right|}^{*} \subseteq \xi_{j, v-1,\left|\rho_{q}\right|}$ (and in fact we have equality, as $m_{v} \uparrow\left|\rho_{q}\right|=$ $\left.m_{v-1} \uparrow\left|\rho_{q}\right|\right)$.

- We have $\left|\rho_{o}\right| \geqslant|\gamma| \geqslant\left|\rho_{q}\right|$. Hence $\xi_{j, v-1,\left|\rho_{q}\right|} \subseteq \xi_{j, v-1,\left|\rho_{o}\right|}$.

- By Proposition 6.7 (2), applied at stage $v-1<s$, we have $\xi_{j, v-1,\left|\rho_{o}\right|}=\eta_{o, j}$. Hence overall we get $\eta_{q, j} \subseteq \eta_{o, j} \subset \zeta_{r}(\delta)$.

Proof of Lemma 6.9, Let $\bar{p}$ be the child of $r$ at stage $s$. If $\bar{p}<p$, then the result follows by induction on $s$, since $\bar{p} \in \operatorname{dom} \mathbb{P}_{s-1}$, and $\eta_{\bar{p}, j} \subset \eta_{p, j}$ (Lemma 6.8). So we assume that $r$ is $p$ 's mother. Let $\delta$ be $p$ 's father. By Lemma 6.20(4), $(r, \delta) \notin \mathbb{P}_{u-1}$.

If $\eta_{p, j}$ is chosen to be an extension of $\xi_{j, s,\left|\rho_{p}\right|}^{*}$, then we note that $\left|\rho_{p}\right|>\left|\rho_{q}\right|$ (Lemma 6.20(2) ); so by Lemma 6.20(3), $\xi_{j, s,\left|\rho_{p}\right|}^{*} \supseteq \xi_{j, s,\left|\rho_{q}\right|}^{*}=\eta_{q, j}$.

Otherwise, $\left(r, \delta^{+}\right) \in \mathbb{P}_{s-1}$, where $\delta^{+}=B_{s} \uparrow|\delta|+1$. We first argue that $|\delta| \geqslant\left|\rho_{q}\right|$. Let $v$ be the stage at which $\delta^{+}$is added to $\operatorname{dom} \alpha_{r}$. Since $(r, \delta) \notin \mathbb{P}_{u-1}$, we have $u<v<s$. We have $\left(r, \delta^{+}\right) \in \mathbb{Q}_{v}$, but $\left(r, \delta^{+}\right) \notin \mathbb{Q}_{s}$ (Lemma 6.18). Hence $m_{s} \uparrow\left|\delta^{+}\right| \neq m_{v} \uparrow\left|\delta^{+}\right|$. As $m_{s}\left\lceil\left|\rho_{q}\right|=m_{v} \uparrow\left|\rho_{q}\right|\right.$, we get $\left|\delta^{+}\right|>\left|\rho_{q}\right|$, so $|\delta| \geqslant\left|\rho_{q}\right|$.

Now if $\eta_{p, j}$ is chosen as an extension of $\xi_{j, s,|\delta|}^{*}$ (if $j \neq j_{r}\left(\rho_{p}\right)$ ), then $\eta_{q, j} \subset \eta_{p, j}$ follows again from Lemma 6.20(3).

Otherwise, $j=j_{r}\left(\rho_{p}\right)$, and $\eta_{p, j}$ is chosen as an extension of $\zeta_{r}\left(\rho_{p}\right)$. The desired result now follows from Lemma 6.20(5).

6.4.5. The consistency of $\Xi_{j}(m)$. We are finally ready to prove that Proposition 6.7 holds at stage $s$, and so complete the induction that started the verification.

Proof of Proposition 6.7. Fix $j<2$. For (1), we need to show that if a new procedure $p$ is called at stage $s$, then the axiom $\mu_{p} \mapsto \eta_{p, j}$ which is enumerated into $\Xi_{j}$ at stage $s$ does not contradict any older axiom in $\Xi_{j}$ which applies to $m_{s}$. Suppose that $\mu_{q} \mapsto \eta_{q, j}$ is an axiom in $\Xi_{j, s-1}$, and that $\mu_{q} \subset m_{s}$; we need to show that $\eta_{q, j} \not \perp \eta_{p, j}$. In fact, $\eta_{q, j} \subset \eta_{p, j}$. For if $q \in \operatorname{dom} \mathbb{P}_{s}$, then $q<p$; by Lemma 6.8, $\eta_{q, j} \subset \eta_{p, j}$. If $q \notin \operatorname{dom} \mathbb{P}_{s}$, then by Lemma 6.9, $\eta_{q, j} \subset \eta_{p, j}$.

For $(2)$, let $p \in \operatorname{dom} \mathbb{P}_{s}$. If $p \in \operatorname{dom} \mathbb{P}_{s-1}$, then $m_{s-1} \uparrow\left|\rho_{p}\right|=m_{s} \uparrow\left|\rho_{p}\right|$. Also, if a new axiom $\mu \mapsto \zeta$ is enumerated into $\Xi_{j}$ at stage $s$, then $|\mu|>\left|\rho_{p}\right|$, as $\mu=\mu_{q}$ for some $q>p$. Hence $\xi_{j, s,\left|\rho_{p}\right|}=\xi_{j, s-1,\left|\rho_{p}\right|}$, and so $\eta_{p, j}=\xi_{j, s,\left|\rho_{p}\right|}$ follows by induction.

Suppose then that $p$ is called at stage $s$. At stage $s$ we enumerate the axiom $\mu_{p} \mapsto \eta_{p, j}$ into $\Xi_{j}$, and so $\eta_{p, j} \subseteq \xi_{j, s,\left|\rho_{p}\right|}$. We have just seen, while proving part (1), that in fact $\Xi_{j, s}\left(m_{s}\right) \subseteq \eta_{p, j}$; that is, if $\mu_{q} \mapsto \eta_{q, j}$ is any axiom in $\Xi_{s}$ which applies to $m_{s}$, then $\mu_{q} \subseteq \mu_{p}$ and $\eta_{q, j} \subseteq \eta_{p, j}$. It follows that $\xi_{j, s,\left|\rho_{p}\right|}=\Xi_{j, s}\left(m_{s}\right)=\eta_{p, j}$.

6.4.6. Consistency of $\Gamma_{j}$. We now work toward our second major goal: showing that for both $j<2$, the functional $\Gamma_{j}$ is consistent. The real reason that $\Gamma_{j}$ is consistent is that when we added a string $\beta^{+}$to $\operatorname{dom} \alpha_{r}$ for some procedure $r$, we 
chose $j=j_{r}\left(\beta^{+}\right)$so that no $\Gamma_{j, s-1^{-a x i o m s}}$ applied to $\sigma \supseteq \alpha^{+}$and $\zeta \subseteq \eta_{q, j}$. How this fact is utilized is a bit involved, though.

From the definition of the functional $\Gamma_{j}$, it is evident that the following lemma is exactly what we need to show that $\Gamma_{j, s}$ is consistent, assuming that $\Gamma_{j, s-1}$ is consistent.

Lemma 6.21. Suppose that $p$ is called at stage $s$; let $q \in \operatorname{dom} \mathbb{P}_{<\omega}$, and let $j<2$. If $\eta_{q, j} \subseteq \eta_{p, j}$, then either $\alpha_{p}\left(\rho_{p}\right) \perp \alpha_{q}\left(\rho_{q}\right)$ or $\rho_{q} \subseteq \rho_{p}$.

We prove Lemma 6.21 using a couple of lemmas which we shortly state. We carry out a "grand induction" similar to the one we used for the consistency of $\Xi_{j}(m)$. We fix a stage $s>0$, and we assume that Lemma 6.21, and all the lemmas that we need for its proof, hold at stage $s-1$; we prove they hold at stage $s$.

The proof of Lemma 6.21 is as follows. Let $p$ and $q$ be as in the statement of the lemma, and let $j<2$. We may assume that $p \neq q$. Since $\left|\eta_{q, j}\right| \leqslant\left|\eta_{p, j}\right|$, and $\eta_{q, j}$ is chosen to be long at the stage at which $q$ is called, assuming that $p \neq q$, we must have $q \in \operatorname{dom} \mathbb{P}_{<s}$.

Let $(r, \beta)=$ parents $(p)$. There are two cases for the choice of $\eta_{p, j}$. If $\eta_{p, j}$ is chosen to be an extension of $\xi_{j, s,\left|\rho_{p}\right|}^{*}$ or of $\xi_{j, s,|\beta|}^{*}$, then let $n=\left|\rho_{p}\right|$ or $n=|\beta|$ accordingly. Since $\eta_{q, j}$ was chosen before stage $s$ and since $\eta_{p, j}$ is a free extension of $\xi_{j, s, n}^{*}$ at stage $s, \eta_{q, j} \subseteq \eta_{p, j}$ implies that $\eta_{q, j} \subseteq \xi_{j, s, n}^{*}$. We have $B_{s}\left\lceil n \subseteq \rho_{p}\right.$ and $A_{s}\left\lceil n \subseteq \alpha_{p}\left(\rho_{p}\right)\right.$. We then use the following lemma:

Lemma 6.22. For all $q \in \operatorname{dom} \mathbb{P}_{<s}$, for all $n<\omega$ and $j<2$, if $\eta_{q, j} \subseteq \xi_{j, s, n}^{*}$, then either $A_{s}\left\lceil n \perp \alpha_{q}\left(\rho_{q}\right)\right.$ or $\rho_{q} \subseteq B_{s}\lceil n$.

Lemma 6.22 implies that either $A_{s} \uparrow n \perp \alpha_{q}\left(\rho_{q}\right)$ or $\rho_{q} \subseteq B_{s} \uparrow n$. Hence either $\alpha_{p}\left(\rho_{p}\right) \perp \alpha_{q}\left(\rho_{q}\right)$ or $\rho_{q} \subseteq \rho_{p}$, verifying Lemma 6.21 in this case.

Otherwise, for $\beta^{+}=B_{s} \uparrow|\beta|+1$ and $\alpha^{+}=A_{s} \uparrow|\beta|+1$, we have $\rho_{p} \supset \beta^{+}$, $\left|\rho_{p}\right|=k_{r}\left(\beta^{+}\right)$, and $j=j_{r}\left(\beta^{+}\right)$. We have $\alpha_{p}\left(\rho_{p}\right) \supseteq \neg \alpha_{r}\left(\beta^{+}\right)$, and $\eta_{p, j}$ is chosen to be a free extension of $\zeta_{r}\left(\rho_{p}\right)$. As before, the assumption $\eta_{q, j} \subseteq \eta_{p, j}$ implies that $\eta_{q, j} \subseteq \zeta_{r}\left(\rho_{p}\right)$. Now we use the following lemma:

Lemma 6.23. Let $\left(r, \beta^{+}\right) \in \mathbb{P}_{s}$, with $\beta^{+} \neq \rho_{r}$. Let $j=j_{r}\left(\beta^{+}\right)$. Let $\delta$ be an extension of $\beta^{+}$of length $k_{r}\left(\beta^{+}\right)$. For all $q \in \operatorname{dom} \mathbb{P}_{<s}$, if $\eta_{q, j} \subseteq \zeta_{r}(\delta)$, then either $\rho_{q} \subset \beta^{+}$or $\alpha_{q}\left(\rho_{q}\right) \perp \neg\left(\alpha_{r}\left(\beta^{+}\right)\right)$.

Using Lemma 6.23, we see that either $\neg \alpha_{r}\left(\beta^{+}\right) \perp \alpha_{q}\left(\rho_{q}\right)$, whence $\alpha_{p}\left(\rho_{p}\right) \perp$ $\alpha_{q}\left(\rho_{q}\right)$, or $\rho_{q} \subset \beta^{+} \subset \rho_{p}$. The proof of Lemma 6.21 is thus complete, once we prove Lemmas 6.22 and 6.23 .

We start with the former.

Proof of Lemma 6.22. Suppose that $\eta_{q, j} \subseteq \xi_{j, s, n}^{*}$. Then there is some $\bar{p} \in \operatorname{dom} \mathbb{P}_{<s}$ such that $\mu_{\bar{p}} \subset m_{s}$ and such that $\left|\rho_{\bar{p}}\right| \leqslant n$ and $\eta_{q, j} \subseteq \eta_{\bar{p}, j}$. By the assumption that Lemma 6.21 holds before stage $s$, either $\alpha_{\bar{p}}\left(\rho_{\bar{p}}\right) \perp \alpha_{q}\left(\rho_{q}\right)$ or $\rho_{q} \subseteq \rho_{\bar{p}}$. In the first case, we note that $\mu_{\bar{p}} \subset m_{s}$ implies that $\alpha_{\bar{p}}\left(\rho_{\bar{p}}\right) \subset A_{s}$, so $A_{s}\left\lceil n \supseteq \alpha_{\bar{p}}\left(\rho_{\bar{p}}\right)\right.$. In the second case, we note that $\mu_{\bar{p}} \subset m_{s}$ implies that $\rho_{\bar{p}} \subset B_{s}$, so $\rho_{q} \subseteq \rho_{\bar{p}} \subseteq B_{s}\lceil n$.

We now work toward the proof of Lemma 6.23.

Lemma 6.24. Let $r \in \operatorname{dom} \mathbb{P}_{s}$, and suppose that a string $\beta^{+} \neq \rho_{r}$ is added to $\operatorname{dom} \alpha_{r}$ at stage s. Let $\beta=\left(\beta^{+}\right)^{-}$be $\beta^{+}$'s immediate predecessor on $\operatorname{dom} \alpha_{r}$. Let 
$p$ be the child of $r$ at stage $s-1$. Let $\delta$ be an extension of $\beta^{+}$of length $k_{r}\left(\beta^{+}\right)$. Let $j=j_{r}\left(\beta^{+}\right)$. Then:

(1) $(r, \beta)=\operatorname{parents}(p)$, and $\alpha_{r}\left(\beta^{+}\right) \subseteq \alpha_{p}\left(\rho_{p}\right)$.

(2) If $(\sigma, \zeta) \mapsto \gamma$ is an axiom in $\Gamma_{j, s-1}, \zeta \subseteq \zeta_{r}(\delta)$, and $\sigma \nsupseteq \alpha_{r}\left(\beta^{+}\right)$, then $\zeta \subseteq \eta_{p, j}$

(3) For every string $\sigma$ comparable with $\neg\left(\alpha_{r}\left(\beta^{+}\right)\right), \Gamma_{j, s-1}\left(\sigma, \zeta_{r}(\delta)\right) \subseteq \beta$.

Proof. We note that $(r, \beta)$ is in both $\mathbb{P}_{s-1}$ and $\mathbb{Q}_{s}$, that $\beta^{+}=B_{s} \uparrow|\beta|+1$, and that $\left(r, \beta^{+}\right) \in \mathbb{Q}_{s}$. At stage $s$, we choose $\zeta_{r}(\delta) \supset \eta_{p, j}$.

That $(r, \beta)=\operatorname{parents}(p)$ follows from Lemma 6.20(1) (using $t=s-1$ and $p$ for $\bar{q}$, so $u=s$ ), which states that if $\gamma$ is $p$ 's father, then a one-bit extension $\gamma^{+}$is added to dom $\alpha_{r}$ at stage $s$; so $\gamma^{+}=\beta^{+}$, so $\gamma=\beta$.

The instructions at stage $s$ say that $\mu_{p} \subset m_{s}$. This implies that $\alpha_{p}\left(\rho_{p}\right) \subset A_{s}$. Since $\alpha_{r}\left(\beta^{+}\right) \subset A_{s}$ and $\left|\rho_{p}\right|>|\beta|$ (Corollary [6.13), from $\left|\alpha_{r}\left(\beta^{+}\right)\right|=|\beta|+1$ and $\left|\rho_{p}\right|=\left|\alpha_{p}\left(\rho_{p}\right)\right|$ we get $\alpha_{r}\left(\beta^{+}\right) \subseteq \alpha_{p}\left(\rho_{p}\right)$. So (1) holds.

We prove part (2). Let $(\sigma, \zeta) \mapsto \gamma$ be an axiom in $\Gamma_{j, s-1}$, and suppose that $\zeta \subseteq \zeta_{r}(\delta)$ and $\sigma \nsupseteq \alpha_{r}\left(\beta^{+}\right)$. Since $\eta_{p, j} \subset \zeta_{r}(\delta), \zeta$ and $\eta_{p, j}$ are comparable; we need to show that $|\zeta| \leqslant\left|\eta_{p, j}\right|$. Suppose otherwise.

Let $q$ be the procedure such that $\sigma=\alpha_{q}\left(\rho_{q}\right), \zeta=\eta_{q, j}$, and $\gamma=\rho_{q}$. So $\left|\eta_{p, j}\right|<$ $\left|\eta_{q, j}\right|$.

Let $t<s$ be the stage at which $p$ was called. Since $\eta_{p, j}$ is chosen long at stage $t$, we get that $q$ is called at a stage $u \in(t, s)$. Since $p$ is in both $\operatorname{dom} \mathbb{P}_{t}$ and in $\operatorname{dom} \mathbb{P}_{s-1}$, we have $p \in \operatorname{dom} \mathbb{P}_{u}$, so $p<q$. This implies that $\alpha_{p}\left(\rho_{p}\right) \subset \alpha_{q}\left(\rho_{q}\right)$ (Lemma 6.16). Since $\alpha_{r}\left(\beta^{+}\right) \subseteq \alpha_{p}\left(\rho_{p}\right)$, we get $\alpha_{r}\left(\beta^{+}\right) \subseteq \alpha_{q}\left(\rho_{q}\right)=\sigma$, contradicting our assumptions.

We turn to part (3). Let $\sigma$ be any string comparable with $\neg\left(\alpha_{r}\left(\beta^{+}\right)\right)$. Let $\gamma=\Gamma_{j, s-1}\left(\sigma, \zeta_{r}(\delta)\right)$. Let $\bar{\sigma} \subseteq \sigma$ and $\zeta \subseteq \zeta_{r}(\delta)$ be strings such that the axiom $(\bar{\sigma}, \zeta) \mapsto \gamma$ is in $\Gamma_{j, s-1}$. The string $\bar{\sigma}$ is comparable with $\neg\left(\alpha_{r}\left(\beta^{+}\right)\right)$, and so does not extend $\alpha_{r}\left(\beta^{+}\right)$. By part (2), $\zeta \subseteq \eta_{p, j}$.

By the choice of $j$ at stage $s$, we know that there are no $\sigma^{\prime} \supseteq \neg\left(\alpha_{r}\left(\beta^{+}\right)\right)$and $\zeta^{\prime} \subseteq \eta_{p, j}$ such that $\left(\sigma^{\prime}, \zeta^{\prime}\right) \in \operatorname{dom} \Gamma_{j, s}$. Hence we must have $\bar{\sigma} \subset \neg\left(\alpha_{r}\left(\beta^{+}\right)\right)$, that is, $\bar{\sigma} \subseteq \alpha_{r}(\beta)$.

The axiom $\left(\alpha_{p}\left(\rho_{p}\right), \eta_{p, j}\right) \mapsto \rho_{p}$ is in $\Gamma_{j, s-1}$, and is compatible with the axiom $(\bar{\sigma}, \zeta) \mapsto \gamma$. By our assumption that $\Gamma_{j, s-1}$ is consistent, we have $\gamma \not \perp \rho_{p}$. As $\bar{\sigma} \subseteq \alpha_{r}(\beta)$, we have $|\gamma|=|\bar{\sigma}| \leqslant|\beta|$. Hence $\gamma \subseteq \rho_{p}|| \beta \mid=\beta$.

Proof of Lemma 6.23. Let $u$ be the stage at which $q$ is called, and let $t$ be the stage at which $\beta^{+}$is added to $\operatorname{dom} \alpha_{r}$. Since $\zeta_{r}(\delta)$ is chosen at stage $t$, and $\eta_{q, j}$ is chosen to be long at stage $u$, the assumption that $\eta_{q, j} \subseteq \zeta_{r}(\delta)$ implies that $u<t$. If $t<s$, then the conclusion holds because, by induction, the lemma holds at stage $s-1$. Suppose then that $t=s$, that is, $\beta^{+}$is added to $\operatorname{dom} \alpha_{r}$ at stage $s$.

The axiom $\left(\alpha_{q}\left(\rho_{q}\right), \eta_{q, j}\right) \mapsto \rho_{q}$ is in $\Gamma_{j, s-1}$. Suppose that $\alpha_{q}\left(\rho_{q}\right)$ is comparable with $\neg\left(\alpha_{r}\left(\gamma^{+}\right)\right)$. Lemma 6.24(3), applied to $\sigma=\alpha_{q}\left(\rho_{q}\right)$, says that

$$
\rho_{q} \subseteq \Gamma_{j, s-1}\left(\alpha_{q}\left(\rho_{q}\right), \zeta_{r}(\delta)\right) \subset \beta^{+},
$$

as required.

6.4.7. The main lemma. We now turn to our third main goal, which is the proof of the main lemma. For brevity, for any $s<\omega$, let $\xi_{j, s}=\Xi_{j, s}\left(m_{s}\right)=\bigcup_{k} \xi_{j, s, k}$ and $\xi_{j, s}^{*}=\Xi_{j, s-1}\left(m_{s}\right)=\bigcup_{k} \xi_{j, s, k}^{*}$. 
Lemma 6.25. Let $s<\omega$. For every $k>0$ there is some $j<2$ such that there are no $\sigma \supseteq \neg\left(A_{s} \uparrow k\right)$ and $\zeta \subseteq \xi_{j, s}$ such that $(\sigma, \zeta) \in \operatorname{dom} \Gamma_{j, s}$.

The main lemma allows us to prove the following corollary, which tells us that if some procedure $p$ requires attention, then we can find some $j<2$ for which there are no disturbing historical $\Gamma_{j, s-1}$ axioms involving $\neg\left(\alpha^{+}\right)$and $\eta_{q, j}$.

Corollary 6.26. Let $s>0$. Suppose that $q \neq p_{0}, q \in \operatorname{dom} \mathbb{P}_{s-1}$ and $\mu_{q} \subset m_{s}$. Let $(p, \beta)=\operatorname{parents}(q)$; let $\beta^{+}=B_{s} \uparrow|\beta|+1$ and $\alpha^{+}=A_{s} \uparrow|\beta|+1$. Then there is some $j<2$ such that there are no $\sigma \supseteq \neg\left(\alpha^{+}\right)$and $\zeta \subseteq \eta_{q, j}$ such that $(\sigma, \zeta) \in \operatorname{dom} \Gamma_{j, s-1}$.

Proof. Let $k=|\beta|+1$; so $\alpha^{+}=A_{s}\left\lceil k\right.$. Since $q \in \operatorname{dom} \mathbb{P}_{s-1}$ and $\mu_{q} \subset m_{s}$, we have, for both $j<2, \eta_{q, j} \subseteq \xi_{j, s}^{*} \subseteq \xi_{j, s}$. The result now follows from the main Lemma 6.25 for $k$.

Toward proving the main lemma, we investigate a third scenario (akin to Lemmas 6.20 and 6.24).

Lemma 6.27. Suppose that a procedure $p$ is called at a stage $s>0$. Let $(r, \gamma)=$ $\operatorname{parents}(p)$ and $\gamma^{+}=B_{s} \uparrow|\gamma|+1$. Suppose that $\left(r, \gamma^{+}\right) \in \mathbb{P}_{s-1}$. Let $t<s$ be the stage at which $\gamma^{+}$was added to $\operatorname{dom} \alpha_{r}$.

(1) $|\gamma|$ is the greatest $n$ such that $A_{t}\left\lceil n=A_{s}\lceil n\right.$.

(2) If $q \in \operatorname{dom} \mathbb{P}_{<s}$ and $\mu_{q} \subset m_{s}$, then $q \in \operatorname{dom} \mathbb{P}_{<t}$.

(3) Let $j<2$. Suppose that $(\sigma, \zeta) \in \operatorname{dom} \Gamma_{j, s-1}$ and either:

- $\zeta \subseteq \xi_{j, t} ;$ or

- $\zeta \subseteq \zeta_{r}(\delta)$ for some $\delta \supset \gamma^{+}$of length $k_{r}\left(\gamma^{+}\right)$.

Then $(\sigma, \zeta) \in \operatorname{dom} \Gamma_{j, t}$.

Proof. By definition, we have $\alpha_{r}\left(\gamma^{+}\right) \subset A_{t}$; hence $\alpha_{r}(\gamma) \subset A_{t}$. Since $\left(r, \gamma^{+}\right) \notin \mathbb{Q}_{s}$, and $\gamma^{+} \subset B_{s}$, we have $\alpha_{r}\left(\gamma^{+}\right) \not \subset A_{s}$. On the other hand, $\alpha_{r}(\gamma) \subset A_{s}$. This establishes (1).

We turn to prove (2). Let $q \in \operatorname{dom} \mathbb{P}_{<s}$ and suppose that $\mu_{q} \subset m_{s}$. Again, as $\gamma^{+} \subset B_{s}$ and $\neg\left(\alpha_{r}\left(\gamma^{+}\right)\right) \subset A_{s}$, let $u$ be the least stage such that for all $v \in[u, s]$ we have $\gamma^{+} \subset B_{v}$ and $\neg\left(\alpha_{r}\left(\gamma^{+}\right)\right) \subset A_{v}$. We have $u>t$ because $\alpha_{r}\left(\gamma^{+}\right) \subset A_{t}$.

The minimality of $u$ implies that $m_{u-1}\left\lceil|\gamma|+1 \neq m_{u}\lceil|\gamma|+1\right.$.

The procedure $r$ is the weakest procedure in $\operatorname{dom} \mathbb{P}_{t}$. We show that if $q$ is a child of $r$ which is called at some stage $v \in(t, s)$, then $\mu_{q} \not \subset m_{s}$.

Let $q$ be such a child. There are two cases.

First suppose that $v<u$. Let $\beta$ be the father of $q$. By Lemma 6.18, $\beta$ is the longest string such that $(r, \beta) \in \mathbb{Q}_{v}$. Now $\gamma$ is the longest string such that $(r, \gamma) \in \mathbb{Q}_{u}$. Hence, if $\beta \neq \gamma$, then $m_{v} \uparrow|\beta|+1 \neq m_{u} \uparrow|\beta|+1$. If $\beta=\gamma$, then we already concluded that $m_{u-1} \uparrow|\beta|+1 \neq m_{u} \uparrow|\beta|+1$; since $v<u$, we get $m_{v} \uparrow|\beta|+1 \neq m_{u} \uparrow|\beta|+1$. Now $\left|\mu_{q}\right|>|\beta|$ (Lemma 6.12), and $\mu_{q} \subset m_{v}$, so $\mu_{q} \not \subset m_{u}$. Hence $\mu_{q} \not \subset m_{s}$.

The other case is that $v \geqslant u$. In this case we know that the father of $q$ is $\gamma$. Since $p$ is a child of $r$ which is called at stage $s$, and $v<s$, we know that $q$ must be cancelled at a stage $w \in(v, s]$. Since $w \geqslant u$, we know that $B_{w} \uparrow|\gamma|+1=\gamma^{+}$, and since $w>t$, we know that $\left(r, \gamma^{+}\right) \in \mathbb{P}_{w-1}$. So it is impossible that $q$ is cancelled at step 2 of stage $w$ : such a cancellation can only occur in order to add $\gamma^{+}$to $\operatorname{dom} \alpha_{r}$. Hence $\mu_{q} \not \subset m_{w}$, so $\mu_{q} \not \subset m_{s}$. 
This establishes (2), which implies that for both $j<2, \xi_{j, s}^{*} \subseteq \xi_{j, t}$. We turn to (3). Let $q$ be any procedure which is called at a stage $v \in(t, s)$. The string $\eta_{q, j}$ is chosen to be a free extension at stage $v$. Both $\left|\xi_{j, t}\right|$ and $\left|\zeta_{r}(\delta)\right|$ are bounded by \# $(t)$. Hence we cannot have $\eta_{q, j} \subseteq \xi_{j, t}$ or $\eta_{q, j} \subseteq \zeta_{r}(\delta)$.

Lemma 6.28. Let $p \in \operatorname{dom} \mathbb{P}_{<\omega}$ and $j<2$. There are no $\sigma \supset \alpha_{p}\left(\rho_{p}\right)$ and $\zeta \subseteq \eta_{p, j}$ such that $(\sigma, \zeta) \in \operatorname{dom} \Gamma_{j}$.

Proof. Let $(\sigma, \zeta) \in \operatorname{dom} \Gamma_{j}$; there is some $q \in \operatorname{dom} \mathbb{P}_{<\omega}$ such that $\sigma=\alpha_{q}\left(\rho_{q}\right)$ and $\zeta=\eta_{q, j}$. Suppose that $\zeta \subseteq \eta_{p, j}$. By Lemma 6.21, either $\sigma \perp \alpha_{p}\left(\rho_{p}\right)$ or $\rho_{q} \subseteq \rho_{p}$. In the first case, certainly $\sigma \not \supset \alpha_{p}\left(\rho_{p}\right)$. In the second case, $|\sigma|=\left|\rho_{q}\right| \leqslant\left|\rho_{p}\right|=\left|\alpha_{p}\left(\rho_{p}\right)\right|$, so again we cannot have $\sigma \supset \alpha_{p}\left(\rho_{p}\right)$.

Proof of Lemma 6.25. The lemma is proved by induction on $s$. The lemma clearly holds for $s=0$. Let $s>0$, and suppose the lemma holds at every $t<s$.

We first note that it is sufficient to show, for every $k>0$, that there is some $j<2$ such that there are no $\sigma \supseteq \neg\left(A_{s}\lceil k)\right.$ and $\zeta \subseteq \xi_{j, s}$ such that $(\sigma, \zeta) \in \operatorname{dom} \Gamma_{j, s-1}$. The reason is that such a $(\sigma, \zeta)$ cannot be added to dom $\Gamma_{j}$ at stage $s$, because at stage $s$ we only enumerate axioms such that $\sigma \subset A_{s}$.

If there is a procedure which is called at stage $s$, call that procedure $p$; let $(r, \gamma)=\operatorname{parents}(p)$, and let $\gamma^{+}=B_{s}\lceil|\gamma|+1$.

There are three cases:

(1) No new procedure is called at stage $s$.

(2) $\left(r, \gamma^{+}\right) \notin \mathbb{P}_{s-1}$.

(3) $\left(r, \gamma^{+}\right) \in \mathbb{P}_{s-1}$.

For each case, we use the inductive hypothesis relative to some previous stage $t$. In cases (1) and (2), let $t=s-1$. In case (3), let $t$ be the stage at which $\gamma^{+}$is added to $\operatorname{dom} \alpha_{r}$.

In all of the cases, let $n$ be the length of the longest common initial segment of $A_{s}$ and $A_{t}$.

Let $k \leqslant n$. Since $A_{t} \uparrow k=A_{s} \uparrow k, \neg\left(A_{s} \uparrow k\right)=\neg\left(A_{t} \uparrow k\right)$. By induction, there is some $j<2$ for which there are no $\sigma \supseteq \neg\left(A_{s}\lceil k)\right.$ and no $\zeta \subseteq \xi_{j, t}$ such that $(\sigma, \zeta) \in \operatorname{dom} \Gamma_{j, t}$. We claim that the same $j$ witnesses the lemma at stage $s$ for $k$. Let $\zeta \subseteq \xi_{j, s}$ and $\sigma \supseteq \neg\left(A_{s}\lceil k)\right.$. We argue that $(\sigma, \zeta) \notin$ dom $\Gamma_{j, s-1}$. Assume, for a contradiction, that $(\sigma, \zeta) \in \operatorname{dom} \Gamma_{j, s-1}$.

In case (1), we have $\xi_{j, s}=\xi_{j, s}^{*} \subseteq \xi_{j, s-1}$, so $\zeta \subseteq \xi_{j, t}$; so by induction, $(\sigma, \zeta) \notin$ $\operatorname{dom} \Gamma_{j, s-1}$.

In case (2), we have $\xi_{j, s}=\eta_{p, j}$. Now $\eta_{p, j}$ is chosen, at stage $s$, to be a free extension of $\xi_{j, s,\left|\rho_{p}\right|}^{*}$. The assumption that $\zeta \subseteq \eta_{p, j}$ and the fact (which we obtained by the assumption, for a contradiction, that $\left.(\sigma, \zeta) \in \operatorname{dom} \Gamma_{j, s-1}\right)$ that $\zeta$ is observed by stage $s-1$, together with the freeness of $\eta_{p, j}$, show that $\zeta \subseteq \xi_{j, s,\left|\rho_{p}\right|}^{*}$, so $\zeta \subseteq \xi_{j, s-1}$. This contradicts the inductive assumption that the lemma holds at stage $s-1$.

In case (3), there are two subcases, depending on the value of $j$. If $j \neq j_{r}\left(\gamma^{+}\right)$, then the argument is similar to that of case (2). We have $\xi_{j, s}=\eta_{p, j}$ is chosen as a free extension of $\xi_{j, s,|\gamma|}^{*}$; the contradictory assumption that $\zeta$ is observed by stage $s-1$ implies that $\zeta \subseteq \xi_{j, s,|\gamma|}^{*} \subseteq \xi_{j, s}^{*}$. By Lemma 6.27(2), we have $\xi_{j, s}^{*} \subseteq \xi_{j, t}$, and by Lemma 6.27(3),$(\sigma, \zeta) \in \operatorname{dom} \Gamma_{j, t}$, contradicting the induction assumption.

Suppose that $j=j_{r}\left(\gamma^{+}\right)$. Then $\xi_{j, s}=\eta_{p, j}$ is chosen to be a free extension of $\zeta_{r}(\delta)$, for $\delta=B_{s}\left\lceil k_{r}\left(\gamma^{+}\right)\right.$. Again, the contradiction assumption implies that 
$\zeta \subseteq \zeta_{r}(\delta)$. By Lemma 6.27(3),$(\sigma, \zeta) \in \operatorname{dom} \Gamma_{j, t}$. By Lemma 6.27(1), $n=|\gamma|$; so $k \leqslant n$ and $\sigma \supseteq \neg\left(A_{t}\lceil k)\right.$ implies that $\sigma \nsupseteq \alpha_{r}\left(\gamma^{+}\right)$. By Lemma 6.24(2), we have $\zeta \subseteq \eta_{q, j}$, where $q$ is the child of $r$ at stage $t-1$; since $\mu_{q} \subset m_{t}$, we have $\eta_{q, j} \subseteq \xi_{j, t}$. So $\zeta \subseteq \xi_{j, t}$, contradicting the induction assumption.

Now let $k>n$. There is some $j<2$ such that $\xi_{j, s}=\xi_{j, s}^{*}$ or $\xi_{j, s}$ is chosen, at stage $s$, as a free extension of an initial segment of $\xi_{j, s}^{*}$ : both $j<2$ would do in cases (1) and (2), and in case (3), we choose $j \neq j_{r}\left(\gamma^{+}\right)$. We claim that such a $j$ witnesses the lemma at stage $s$ for $k$. Suppose that $\sigma \supseteq \neg\left(A_{s}\lceil k)\right.$. Then $\sigma \supset A_{s}\lceil n$. Let $\zeta \subseteq \xi_{j, s}$, and suppose, for a contradiction, that $(\sigma, \zeta) \in \operatorname{dom} \Gamma_{j, s-1}$. As argued above, the choice of $j$ implies that $\zeta \subseteq \xi_{j, s}^{*}$.

Now let $q \in \operatorname{dom} \mathbb{P}_{<s}$ be a procedure such that $\mu_{q} \subset m_{s}$ and $\eta_{q, j}=\xi_{j, s}^{*}$. We claim that $\left|\mu_{q}\right| \leqslant n$. In cases (1) and (2), this follows from the fact that $\mu_{q} \subseteq m_{s}$ and $m_{s}\left\lceil n+1 \neq m_{s-1}\left\lceil n+1\right.\right.$. In case (3), Lemma 6.27(2) states that $q \in \operatorname{dom} \mathbb{P}_{<t}$, so $\left|\mu_{q}\right| \leqslant n$ follows from the fact that $m_{s}\left\lceil n+1 \neq m_{t}\lceil n+1\right.$.

Now $\left|\mu_{q}\right| \leqslant n$ implies that $\left|\alpha_{q}\left(\rho_{q}\right)\right| \leqslant n ; \mu_{q} \subset m_{s}$ implies that $\alpha_{q}\left(\rho_{q}\right) \subset A_{s}$, so $\alpha_{q}\left(\rho_{q}\right) \subseteq A_{s}\left\lceil n \subset \sigma\right.$. So $\zeta \subseteq \eta_{q, j}$ contradicts Lemma 6.28.

6.4.8. Uncancelled procedures. The following is immediate:

Lemma 6.29. The following are equivalent for $(p, \beta) \in \mathbb{P}_{<\omega}$ :

(1) There are infinitely many s such that $(p, \beta) \in \mathbb{Q}_{s}$.

(2) For almost all $s,(p, \beta) \in \mathbb{Q}_{s}$.

(3) $p$ is never cancelled, $\beta \subset B$ and $\alpha_{p}(\beta) \subset A$.

Let $\mathbb{Q}_{\infty}$ denote the collection of pairs $(p, \beta)$ which satisfy the conditions of Lemma 6.29. We note that if $(p, \beta) \in \mathbb{Q}_{\infty}$, then $(p, \gamma) \in \mathbb{Q}_{\infty}$ for all $\gamma$ such that $\rho_{p} \subseteq \gamma \subseteq \beta$, and that if $p$ is a procedure which is never cancelled, then $\left(p, \rho_{p}\right) \in \mathbb{Q}_{\infty}$.

In this section, let $p$ be some procedure which is never cancelled. Note that if $p \in \operatorname{dom} \mathbb{P}_{s}$, then no procedure stronger than $p$ requires attention at stage $s$, as the strongest such would cancel $p$.

Claim 6.30. There are only finitely many strings $\beta$ such that $(p, \beta) \in \mathbb{Q}_{\infty}$.

Proof. This is because we assume that $A \nless_{T} B$. If the claim fails, then for every $\beta \subset B$ such that $|\beta| \geqslant\left|\rho_{p}\right|$ we have $(p, \beta) \in \mathbb{Q}_{\infty}$, which implies that $\alpha_{p}(\beta) \subset A$; thus $A=\bigcup\left\{\alpha_{p}(\beta): \rho_{p} \subseteq \beta \subset B\right\}$, which shows that $A \leqslant_{T} B$.

Since $p$ is never cancelled, we know that $\left(p, \rho_{p}\right) \in \mathbb{Q}_{\infty}$ (and so $\rho_{p} \subset B$ ). Let $\beta$ be the longest initial segment of $B$ such that $(p, \beta) \in \mathbb{Q}_{\infty}$. Let $\beta^{+}=B\lceil|\beta|+1$.

If $\beta^{+} \in \operatorname{dom} \alpha_{p}$, let $s_{0}$ be a stage such that $\left(p, \beta^{+}\right) \in \mathbb{P}_{s_{0}}$; otherwise, let $s_{0}$ be a stage such that $(p, \beta) \in \mathbb{P}_{s_{0}}$. Also, choose $s_{0}$ sufficiently large such that $m_{s_{0}} \uparrow|\beta|+1 \subset m$.

Claim 6.31. $p$ doesn't require attention after stage $s_{0}$.

Proof. If $\left(p, \beta^{+}\right) \in \mathbb{P}_{<\omega}$, the conclusion follows from the fact that for all $s \geqslant s_{0}$, $\beta^{+}=B_{s} \uparrow|\beta|+1$. If $\left(p, \beta^{+}\right) \notin \mathbb{P}_{<\omega}$, the conclusion follows from the fact that if $p$ required attention at some stage $s \geqslant s_{0}$, then since $(p, \beta) \in \mathbb{Q}_{s}$, it would try to add $B_{s} \uparrow|\beta|+1=\beta^{+}$to $\operatorname{dom} \alpha_{p}$, and then we'd have $\left(p, \beta^{+}\right) \in \mathbb{P}_{<\omega}$, contrary to assumption. 
It follows that for all $s \geqslant s_{0}$, if after step 1 of stage $s, p$ does not have a child, then such a child is called for $p$ at stage $s$. So for all $s \geqslant s_{0}, p$ has a child in $\operatorname{dom} \mathbb{P}_{s}$.

Claim 6.32. There is some child of $p$ which is never cancelled.

Proof. If $\left(p, \beta^{+}\right) \in \mathbb{P}_{<\omega}$, let $k=k_{p}\left(\beta^{+}\right)$. Otherwise, let $k=\#\left(s_{0}\right)+1$. Let $s_{1}>s_{0}$ be a stage such that for all $s \geqslant s_{1}, m_{s}\lceil k \subset m$. If $q$ is a child of $p$ which is called after stage $s_{1}$, then $\left|\rho_{q}\right| \leqslant k$, and so the stability of $m_{s}\left\lceil k\right.$ after stage $s_{1}$ ensures that $q$ is never cancelled.

6.4.9. The end. We know that $p_{0}$ is never cancelled (Lemma 6.10). By recursion, given a procedure $p_{e}$ which is never cancelled, we let, by Claim 6.32, $p_{e+1}$ be the child of $p_{e}$ which is never cancelled. Note that $e_{p_{e}}=e$.

Let $j<2$. Let $X_{j}=\Xi_{j}(m)$. For every $e$, we have $\mu_{p_{e}} \subset m$, so $\eta_{p_{e}, j} \subset X_{j}$. Since $\eta_{p_{e}, j} \subset \eta_{p_{e+1}, j}$, we have $X_{j} \in 2^{\omega}$. We have $X_{j} \leqslant_{T} m \leqslant_{T} C$.

For every $e<\omega$, since $\mu_{p_{e}} \subset m$, we have $\rho_{p_{e}} \subset B$ and $\alpha_{p_{e}}\left(\rho_{p_{e}}\right) \subset A$. Since $\eta_{p_{e}, j} \subset X_{j}$, and the axiom $\left(\alpha_{p_{e}}\left(\rho_{p_{e}}\right), \eta_{p_{e}, j}\right) \mapsto \rho_{p_{e}}$ is in $\Gamma_{j}$, we have $\rho_{p_{e}} \subseteq \Gamma_{j}\left(A, X_{j}\right)$. Since $\left|\rho_{p_{e}}\right|<\left|\rho_{p_{e+1}}\right|$, we have $B=\bigcup_{e} \rho_{p_{e}}$. Hence $B \subseteq \Gamma_{j}\left(A, X_{j}\right)$. Since $\Gamma_{j}$ is consistent, we have $B=\Gamma_{j}\left(A, X_{j}\right)$. Hence $B \leqslant_{T} A \oplus X_{j}$.

To complete the proof of the theorem, we need to show that for all $e$, the requirement $R_{e}$ is met. To see this, we look at two cases. Let $p=p_{e}$; define $\beta, \beta^{+}$ and $s_{0}$ as in the section above. Let $q=p_{e+1}$, and let $s_{1}>s_{0}$ be a stage by which $q$ has been called.

If $\left(p, \beta^{+}\right) \in \mathbb{P}_{<\omega}$, let $j=j_{p}\left(\beta^{+}\right)$. We know that $X_{j} \supset \eta_{q, j} \supset \zeta_{p}\left(\rho_{q}\right)$, that $\beta^{+} \subset B$, and that $\Psi_{e}\left(\zeta_{p}\left(\rho_{q}\right)\right) \perp \beta^{+}$. Hence $\Psi_{e}\left(X_{j}\right) \neq B$.

Now suppose that $\left(p, \beta^{+}\right) \notin \mathbb{P}_{<\omega}$, and suppose, for a contradiction, that the requirement $R_{e}$ fails: $\Psi_{e}\left(X_{0}\right)=\Psi_{e}\left(X_{1}\right)=B$. Since $B$ is not computable, for both $j<2$ there is some string $\tau_{j} \supset \eta_{q, j}$ such that $\Psi_{e}\left(\tau_{j}\right) \perp B$. Again for both $j<2$, let $\sigma_{j} \subset X_{j}$ be a string such that $\sigma_{j} \supset \eta_{q, j}$ and such that $\Psi_{e}\left(\sigma_{j}\right) \perp \Psi_{e}\left(\tau_{j}\right)$. Let $s \geqslant s_{1},\left|\sigma_{0}\right|,\left|\sigma_{1}\right|,\left|\tau_{0}\right|,\left|\tau_{1}\right|$.

By Corollary [6.26, let $j<2$ be such that there are no $\sigma \supseteq \neg\left(A_{s} \uparrow|\beta|+1\right)$ and $\zeta \subseteq \eta_{q, j}$ such that $(\sigma, \zeta) \in \operatorname{dom} \Gamma_{j, s-1}$. Let $k$ be large at stage $s$. Let $\gamma \supset \beta^{+}$have length $k$. If $\gamma \not \perp \Psi_{e}\left(\sigma_{j}\right)$, let $\zeta_{\gamma}=\tau_{j}$; since $|\gamma|=k>\left|\Psi_{e}\left(\tau_{j}\right)\right|$, we have $\Psi_{e}\left(\tau_{j}\right) \perp \gamma$. Otherwise, we let $\zeta_{\gamma}=\sigma_{j}$. Then, in either case, we have $\Psi_{e}\left(\zeta_{\gamma}\right) \perp \gamma$. All the conditions hold for $p$ to require attention at stage $s$, contradicting Claim 6.31.

\section{The JoIn PROPERTY BELOW NON-GENERALIZED-LOW 2 DEGREES}

In this section we prove Theorem 1.7. That is, we show that every non-generalized-low 2 degree has the join property: if $\boldsymbol{d}$ is not $\mathrm{GL}_{2}$ and $\mathbf{0}<\boldsymbol{a}<\boldsymbol{d}$, then there exists $\boldsymbol{b}<\boldsymbol{d}$ such that $\boldsymbol{a} \vee \boldsymbol{b}=\boldsymbol{d}$.

So suppose we are given $D$ of degree which is not $\mathrm{GL}_{2}$, and $A$ which is of nonzero degree strictly below that of $D$. We want to construct $B<_{T} D$ such that $A \oplus B \equiv_{T} D$. Moreover, we will build $B$ to be 1-generic. This will imply that $B \not \equiv_{T} D$, as 1-generic sets are $G L_{1}$.

The general plan is that we construct $B$ by finite approximations, first trying to satisfy a genericity requirement, then coding one bit of $D$, then trying another genericity requirement, and then another bit of $D$, etc. The property of non-GL $\mathrm{GL}_{2}$ sets we will use is the following: For every $D \oplus \emptyset^{\prime}$-computable function $f_{1}$, there is a $D$-computable function $f_{2}$ not dominated by $f_{1}$ (see Ler83]). We will use this function to bound our searches when we are trying to force inside some c.e. set. To 
get $D \leqslant_{T} A \oplus B$, we will have to use a trick, due to Slaman and Steel [SS89]: we will try to satisfy the genericity requirements in a way that can be decoded by $A$ so that $A$ can read off the bits of $B$ that are coding $D$.

Define

$$
\sigma_{n}=\underbrace{000 \cdots}_{n \text { zeros }} 1 .
$$

We can assume that $A$ is not computably enumerable (for instance by considering either it or its complement).

Given any string $\sigma \in 2^{<\omega}$ and any $e, s \in \omega$, we let $n(\sigma, e, s)$ be the least number $n$ such that one of the following two conditions holds:

$n \in A$, and there is no $\tau \in W_{e, s}$ extending $\sigma \frown \sigma_{n}$;

$n \notin A$, and then there is some $\tau \in W_{e, s}$ extending $\sigma \frown \sigma_{n}$.

Note that the set of $n$ such that there is some $\tau \in W_{e, s}$ extending $\sigma \frown \sigma_{n}$ is computable, and hence different from $A$. So, there has to be an $n$ as above, and so $n(\sigma, e, s)$ is well defined. If $n(\sigma, e, s) \in A$, define $g(\sigma, e, s)=\sigma \frown \sigma_{n(\sigma, e, s)}$. If $n(\sigma, e, s) \notin A$, then let $g(\sigma, e, s)$ be the first string $\tau$ enumerated into $W_{e}$ with $\tau \supseteq \sigma^{\frown} \sigma_{n(\sigma, e, s)}$. The functions $n(\sigma, e, s)$ and $g(\sigma, e, s)$ are computable in $A$.

Given any $f: \omega \rightarrow \omega$ we define a set $B_{f}$ that uses the function $f$ to bound the searches of the function $g(\sigma, e, s)$. Let $B_{f}=\bigcup_{s} \tau_{f, s}$ be defined as follows:

Stage 0. Define $\tau_{f, 0}=D(0)$.

Stage $t+1$. Define $\tau_{f, t+1}=g\left(\tau_{f, t}, e, f(t)\right) \frown D(t+1)$, where $t=\langle e, x\rangle$.

Note that $B_{f}$ is computable in $D \oplus f$. We will show later how, if we use a large enough function $f$, we get that $B_{f}$ is 1-generic. That $D$ is computable in $A \oplus B_{f}$ (without appealing to $f$ ) follows using precisely the same argument as originally provided by Slaman and Steel when proving that $\mathbf{0}^{\prime}$ satisfies the join property. The point is that using $B_{f}, A$ can reconstruct the whole sequence $\tau_{f, 0} \subset \tau_{f, 1} \subset \tau_{f, 2} \subset \cdots$ as follows. Given $\tau_{f, k}$, let $n$ be such that $\tau_{f, k} \frown \sigma_{n} \subseteq B_{f}$. If $n \in A$, then we know that $g\left(\tau_{f, k}, e, f(k)\right)=\tau_{f, k} \frown \sigma_{n}$. If $n \notin A$, then $g\left(\tau_{f, k}, e, f(k)\right)$ is the least $\tau \supseteq \tau_{f, k} \frown \sigma_{n}$ enumerated into $W_{e}$; here again we note that we do not need to consult $f$. Then, we get $\tau_{f, k+1}$ by adding, at the end of the string $g\left(\tau_{f, k}, e, f(k)\right)$, the bit $D(k+1)$, which we can read from $B_{f}$. So $D \leqslant_{T} A \oplus B_{f}$.

Given $\sigma$ and $e$, let $n^{*}(\sigma, e)=\lim _{s} n(\sigma, e, s)$ be the least natural number $n$ such that one of the following conditions holds:

$n \in A$, and there is no $\tau \in W_{e}$ extending $\sigma^{\frown} \sigma_{n}$;

$n \notin A$, and there is some $\tau \in W_{e}$ extending $\sigma \frown \sigma_{n}$.

Note that the set of numbers $n$ such that there is some $\tau \in W_{e}$ extending $\sigma^{\frown} \sigma_{n}$ is c.e., and hence different from $A$; so such an $n$ exists, and so $n^{*}(\sigma, e)$ is well defined. We then let $g^{*}(\sigma, e)=\lim _{s} g(\sigma, e, s)$, and let $h^{*}(\sigma, e)$ be the least stage $s$ such that for all $t \geqslant s, g(\sigma, e, s)=g^{*}(\sigma, e)$. The stage $h^{*}(\sigma, e)$ is the least stage $s$ such that:

- if $n^{*}(\sigma, e) \notin A$, then there is some $\tau \in W_{e, s}$ extending $\sigma^{\frown} \sigma_{n^{*}(\sigma, e)}$, and

- for all $k<n^{*}(\sigma, e)$, if $k \in A$, then there is some $\tau \in W_{e, s}$ extending $\sigma \frown \sigma_{k}$.

The functions $n^{*}(\sigma, e), g^{*}(\sigma, e)$ and $h^{*}(\sigma, e)$ are $A \oplus \emptyset^{\prime}$-computable. The string $g^{*}(\sigma, e)$ is an extension of $\sigma$ that either forces inside $W_{e}$ or forces outside $W_{e}$ as we need for the genericity requirements.

For $t \in \omega$ let $\Pi_{t}=\left\{\tau_{f, t} \mid f: \omega \rightarrow \omega\right\}$. This set is finite, and (a canonical index for it) is effectively obtained from $t$ with oracle $D \oplus \emptyset^{\prime}$. This is because given $\tau_{f, t-1}$, 
the possible values for $\tau_{f, t}$ are

$$
\left\{g\left(\tau_{f, t-1}, e, s\right) \frown D(t+1): s \leqslant h^{*}\left(\tau_{f, t-1}, e\right)\right\},
$$

where $t=\langle e, x\rangle$.

So let $f_{0}$ be an increasing function computable in $D \oplus \emptyset^{\prime}$ such that, for all $t$ and all $\tau \in \Pi_{t}, f_{0}(t)$ is greater than $h^{*}(\tau, e)$, where $t=\langle e, x\rangle$. Let $h$ be a computable and increasing function such that, for all $t$ and all $e \leqslant t$, there exists $x$ with $t<\langle e, x\rangle<h(t)$. Define $f_{1}(t)=f_{0}(h(t))$ for all $t$. Since $D$ is not $\mathrm{GL}_{2}$, we may let $f_{2}$ be an increasing function computable in $D$ which is not dominated by $f_{1}$ and then define $B=B_{f_{2}} \leqslant T$.

It remains to show that $B$ is 1-generic. In order to see this, fix $e \in \omega$ and let $t>e$ be such that $f_{2}(t)>f_{1}(t)$. Then there exists $t^{\prime}=\langle e, x\rangle$ such that $t<t^{\prime}<h(t)$. We have that $f_{2}\left(t^{\prime}\right)>f_{2}(t)>f_{1}(t)=f_{0}(h(t))>f_{0}\left(t^{\prime}\right)$, so that $f_{2}\left(t^{\prime}\right)>h^{*}\left(\tau_{f_{2}, t^{\prime}}, e\right)$, as required.

\section{REFERENCES}

[Coo89] S. B. Cooper, The strong anticupping property for recursively enumerable degrees, J. Symbolic Logic 54 (1989), no. 2, 527-539. MR997886 (91b:03071)

[Dow87] R. G. Downey, $\Delta_{2}^{0}$ degrees and transfer theorems, Illinois J. Math. 31 (1987), no. 3, 419-427. MR892177 (89c:03070)

[Eps79] Richard L. Epstein, Degrees of unsolvability: structure and theory, Lecture Notes in Mathematics, vol. 759, Springer, Berlin, 1979. MR551620(81e:03042)

[Fri57] Richard M. Friedberg, Two recursively enumerable sets of incomparable degrees of unsolvability (solution of Post's problem, 1944), Proc. Nat. Acad. Sci. U.S.A. 43 (1957), 236-238. MR0084474(18,867a)

[Joc80] Carl G. Jockusch, Jr., Degrees of generic sets, Recursion Theory: its Generalisation and Applications (Proc. Logic Colloq., Univ. Leeds, Leeds, 1979), London Math. Soc. Lecture Note Ser., vol. 45, Cambridge Univ. Press, Cambridge, 1980, pp. 110-139. MR83i:03070

[JS83] Carl G. Jockusch, Jr. and Richard A. Shore, Pseudojump operators. I. The r.e. case, Trans. Amer. Math. Soc. 275 (1983), no. 2, 599-609. MR682720 (84c:03081)

[JS93] C.G. Jockusch Jr. and T.A. Slaman, On the $\Sigma_{2}$ theory of the upper semilattice of the Turing degrees, Journal of Symbolic Logic 58 (1993), 193-204. MR1217184|(94d:03084)

[KP54] S.C. Kleene and E.L. Post, The upper semi-lattice of the degrees of recursive unsolvability, Annals of Mathematics (2) 59 (1954), 379-407. MR0061078 (15:772a)

[Ler83] M. Lerman, Degrees of unsolvability, Springer-Verlag, Berlin, Heidelberg, New York, Tokyo, 1983. MR708718(85h:03044)

[Lew05] Andrew E. M. Lewis, The minimal complementation property above 0', MLQ Math. Log. Q. 51 (2005), no. 5, 470-492. MR2163759 (2006d:03066)

[LL76] A. H. Lachlan and R. Lebeuf, Countable initial segments of the degrees of unsolvability, J. Symbolic Logic 41 (1976), no. 2, 289-300. MR0403937 (53:7746)

[LS75] Richard E. Ladner and Leonard P. Sasso, Jr., The weak truth table degrees of recursively enumerable sets, Ann. Math. Logic 8 (1975), no. 4, 429-448. MR0379157 (52:63)

[LS88] Manuel Lerman and Richard A. Shore, Decidability and invariant classes for degree structures, Trans. Amer. Math. Soc. 310 (1988), no. 2, 669-692. MR973174(90c:03031)

[MNS04] Russell G. Miller, Andre O. Nies, and Richard A. Shore, The $\forall \exists$-theory of $\mathcal{R}(\leqslant, \vee, \wedge)$ is undecidable, Trans. Amer. Math. Soc. 356 (2004), no. 8, 3025-3067 (electronic). MR2052940 (2005g:03057)

[Mon] Antonio Montalbán, Embeddings into the Turing degrees, Lect. Notes Log., Vol. 32, Assoc. Symbol. Logic, Chicago, IL, 2009. MR2562555 (2011d:03063)

[Muc56] A. A. Muchnik, On the unsolvability of the problem of reducibility in the theory of algorithms, Dokl. Akad. Nauk SSSR, N.S. 108 (1956), 194-197. MR0081859 (18:457a)

[Pos77] David Posner, High degrees, Ph.D. thesis, University of California, Berkeley, 1977. MR 2627247

[PR81] D. B. Posner and R. W. Robinson, Degrees joining to 0', J. Symbolic Logic 46 (1981), no. 4, 714-722. MR83c:03040 
[Rob72] R. W. Robinson, Degrees joining 0', Notices of the American Mathematical Society 19 (1972), A-615.

[Sac61] Gerald E. Sacks, A minimal degree less than 0', Bull. Amer. Math. Soc. 67 (1961), 416-419. MR0126380(23:A3676)

[Sac63] _ On the degrees less than 0', Ann. of Math. (2) 77 (1963), 211-231. MR.0146078 $(26: 3604)$

[Sho59] J. R. Shoenfield, On degrees of unsolvability, Ann. of Math. (2) 69 (1959), 644-653. MR0105355 (21:4097)

[Sho81] Richard A. Shore, The theory of the degrees below 0', J. London Math. Soc. 24 (1981), 1-14. MR623666 (83m:03051)

[Sho06] Richard A. Shore, Degree structures: Local and global investigations, Bulletin of Symbolic Logic 12 (2006), 369-389. MR2248589 (2007e:03074)

[Soa87] Robert I. Soare, Recursively enumerable sets and degrees, Perspectives in Mathematical Logic, Springer-Verlag, Berlin, 1987, A study of computable functions and computably generated sets. MR88m:03003

[SS89] T. A. Slaman and J. R. Steel, Complementation in the Turing degrees, J. Symbolic Logic 54 (1989), no. 1, 160-176. MR90c:03034

[SS01] Theodore A. Slaman and Robert I. Soare, Extension of embeddings in the computably enumerable degrees, Ann. of Math. (2) 154 (2001), no. 1, 1-43. MR.1847587 (2002f:03079)

School of Mathematics, Statistics and Computer Science, Victoria University, P.O. Box 600, Wellington, New Zealand

E-mail address: Rod.Downey@msor.vuw.ac.nz

School of Mathematics, Statistics and Computer Science, Victoria University. P.O. Box 600, Wellington, New Zealand

E-mail address: greenberg@msor.vuw.ac.nz

School of Mathematics, University of Leeds, Leeds, United Kingdom

E-mail address: andy@aemlewis.co.uk

Department of Mathematics, University of Chicago, 5734 S. University Ave., Chicago, ILLINOIS 60637

E-mail address: antonio@math.uchicago.edu 